\title{
A Spatially Distributed Investigation of Stream Water Temperature in a Contemporary Mixed-Land-Use Watershed
}

Jason P. Horne

West Virginia University

Follow this and additional works at: https://researchrepository.wvu.edu/etd

Part of the Environmental Health and Protection Commons, Environmental Monitoring Commons, Hydrology Commons, and the Water Resource Management Commons

\section{Recommended Citation}

Horne, Jason P., "A Spatially Distributed Investigation of Stream Water Temperature in a Contemporary Mixed-Land-Use Watershed" (2020). Graduate Theses, Dissertations, and Problem Reports. 7741.

https://researchrepository.wvu.edu/etd/7741

This Thesis is protected by copyright and/or related rights. It has been brought to you by the The Research Repository @ WVU with permission from the rights-holder(s). You are free to use this Thesis in any way that is permitted by the copyright and related rights legislation that applies to your use. For other uses you must obtain permission from the rights-holder(s) directly, unless additional rights are indicated by a Creative Commons license in the record and/ or on the work itself. This Thesis has been accepted for inclusion in WVU Graduate Theses, Dissertations, and Problem Reports collection by an authorized administrator of The Research Repository @ WVU. For more information, please contact researchrepository@mail.wvu.edu. 


\section{A Spatially Distributed Investigation of Stream Water Temperature in a Contemporary Mixed-Land-Use Watershed}

Jason P. Horne

Follow this and additional works at: https://researchrepository.wvu.edu/etd

Part of the Environmental Health and Protection Commons, Environmental Monitoring Commons, Hydrology Commons, and the Water Resource Management Commons 


\title{
A Spatially Distributed Investigation of Stream Water Temperature in a Contemporary Mixed-Land-Use Watershed
}

\author{
Jason Horne \\ Thesis submitted to the \\ Davis College of Agriculture, Natural Resources and Design \\ at West Virginia University
}

in partial fulfillment of the requirements for the degree of

Master of Science in

Environmental, Soil and Water Sciences

Jason A. Hubbart, Ph.D., Chair

Elliott Kellner, Ph.D.

Kirsten Stephan, Ph.D.

Division of Plant and Soil Sciences

Morgantown, West Virginia

2020

Keywords: stream water temperature; land use; hydrology; experimental watershed (c) Copyright by Jason P. Horne 2020 


\section{ABSTRACT \\ A Spatially Distributed Investigation of Stream Water Temperature in a Contemporary Mixed-Land-Use Watershed}

\section{Jason Horne}

Stream water temperature is an important physical variable that influences many biological and abiotic water quality processes. The land-use/land-cover (LULC) types and corresponding variability in stream water temperature $(\mathrm{Tw})$ processes in contemporary mixed-land-use watersheds necessitate research to advance management and policy decisions. Water temperature was analyzed from 21 gauging sites using a nested-scale experimental watershed study design. Results showed that forested land use was significantly negatively correlated $(\alpha=0.05)$ with mean and maximum Tw. Agricultural land use was significantly positively correlated $(\alpha=0.05)$ with maximum Tw except during the spring season. Mixed development and Tw were significantly correlated $(\alpha=0.05)$ at quarterly and monthly timescales. Correlation trends in some reaches were reversed between the winter and summer seasons, contradicting previous research. During the winter season, mixed development showed a negative relationship with minimum Tw and mean Tw. During the summer season, higher minimum, maximum, and mean Tw correlations were observed relative to the winter season. Advanced understanding generated through this high-resolution investigation improves land managers' ability to improve conservation strategies in freshwater aquatic ecosystems of contemporary watersheds. 
The undersigned, appointed by the dean of the Graduate School, have examined the thesis entitled

A Spatially Distributed Investigation of Stream Water Temperature in a Contemporary

Mixed-Land-Use Watershed

presented by Jason P. Horne,

a candidate for the degree of Master of Science,

and hereby certify that, in their opinion, it is worthy of acceptance.

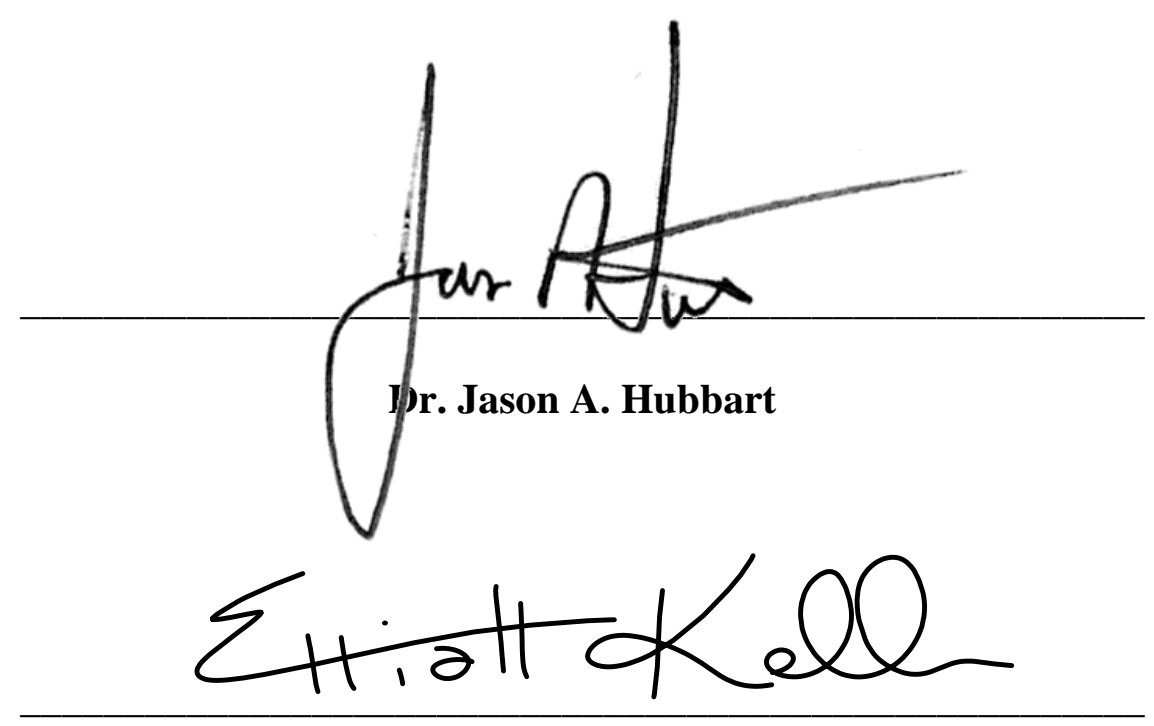

Dr. Elliott Kellner

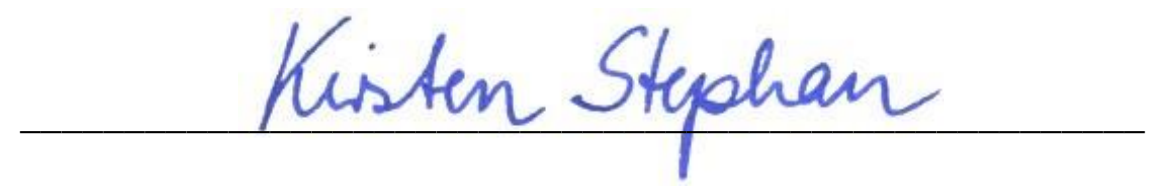

Dr. Kirsten Stephan

iii 


\section{ACKNOWLEDGMENTS}

I would like to thank my major advisor, Dr. Jason A. Hubbart, for the time and effort devoted to my development as not only a student but also a scientist. Throughout the entire process his valuable comments and insights were instrumental in locating and strengthening my many weaknesses. He is and forever will be an instrumental part of my progression into the world of academia and I am grateful for the opportunity he has given me.

I would also like to thank my committee members, Dr. Kirsten Stephan and Dr. Elliot Kellner for the time they have given in both reading my work and providing valuable feedback. The skills and expertise they bring to the table were crucial in this project's success.

I would like to thank all the federal and state agencies that provided funding for this project including the National Science Foundation under Award Number OIA-1458952, the USDA National Institute of Food and Agriculture, Hatch project accession number 1011536, and the West Virginia Agricultural and Forestry Experiment Station. This project was possible thanks to their contributions through financial support.

Finally, I would like to thank my colleagues in the Interdisciplinary Hydrology Laboratory, Zack Heck and Fritz Petersen, for the countless hours of devotion to fieldwork and friendship. This would not have been possible without their help throughout this entire process. 


\section{TABLE OF CONTENT}

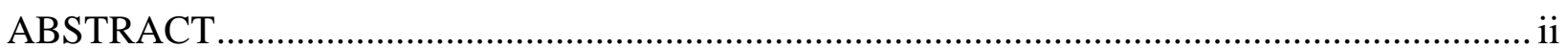

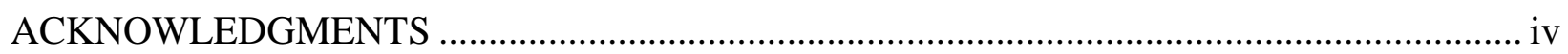

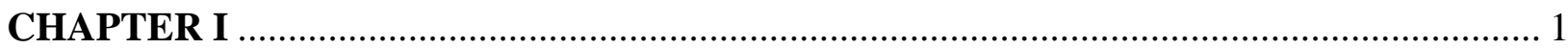

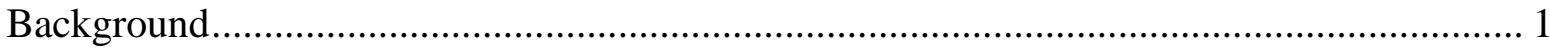

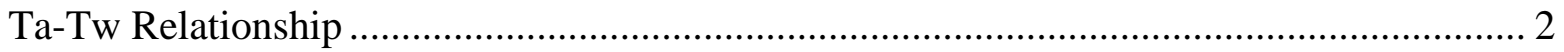

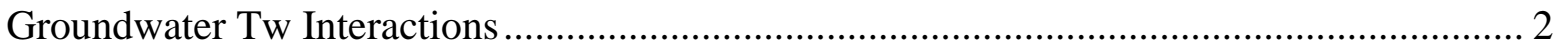

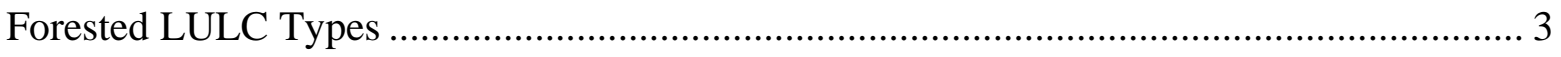

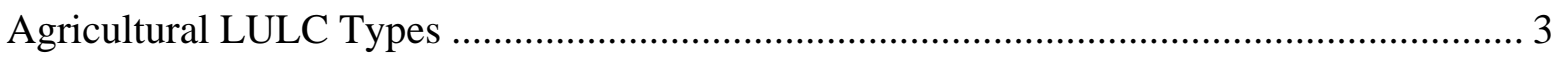

Mixed Development LULC Types ................................................................................. 4

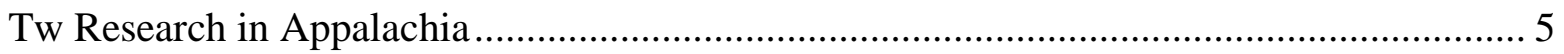

The Nested Experimental Watershed Study Design............................................................. 5

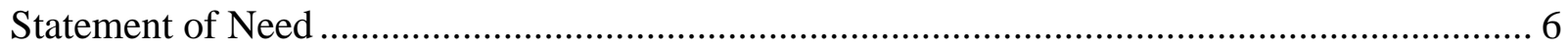

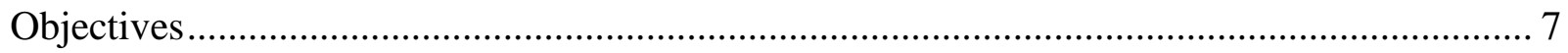

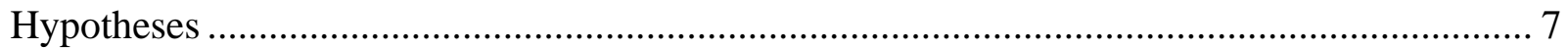

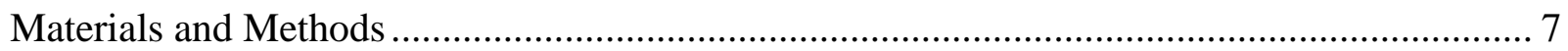

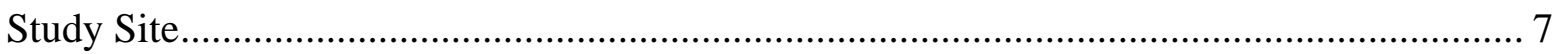

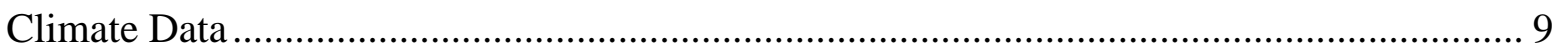

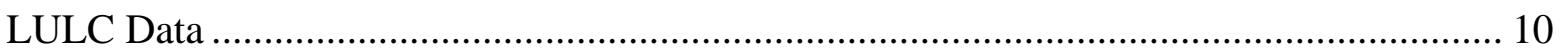

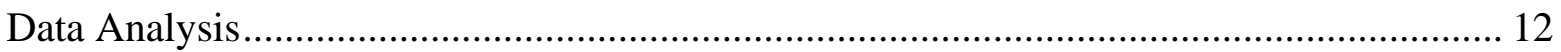

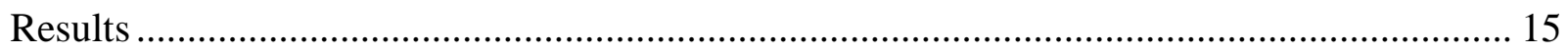

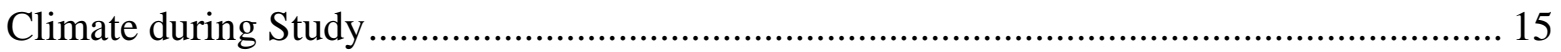

Annual Stream Water Temperature ……………………………………………………….... 17

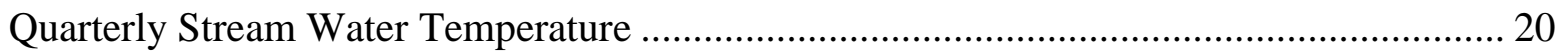

Monthly Stream Water Temperature ………………….................................................... 22

PCA … 


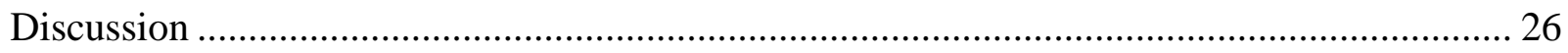

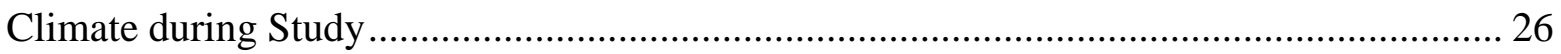

Stream Water Temperature LULC Relations ............................................................ 26

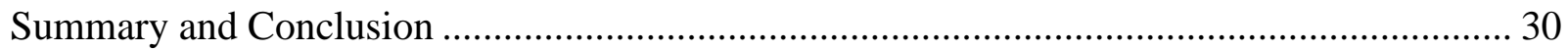

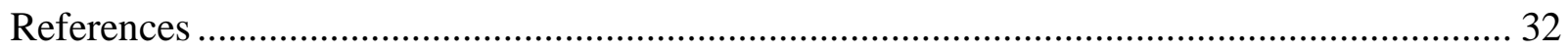

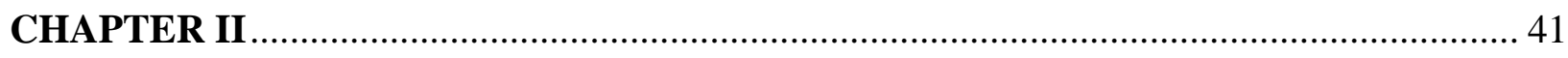

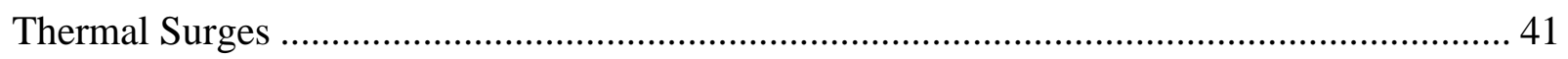

Relationship of Stream Distance and Tw ................................................................... 44

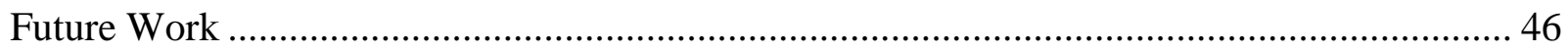

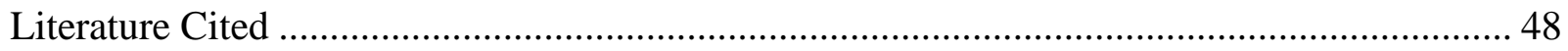

APPENDIX A

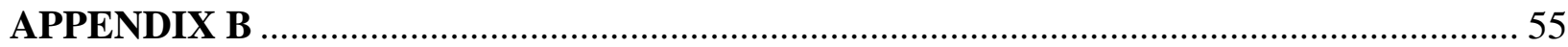

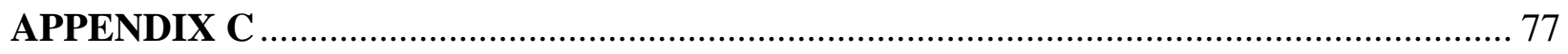

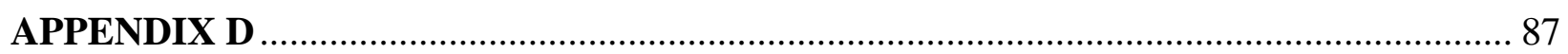

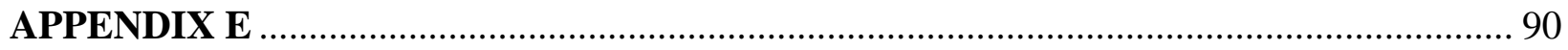




\section{LIST OF FIGURES}

Figure Page

Figure 1. Land use and land cover of West Run Watershed (WRW), West Virginia, USA including 22 nested gauging sites and corresponding sub-basins.....

Figure 2. (a) Proportion LULC of sub-catchment and associated sites, (b) LULC area of subcatchment and each associated site in West Run Watershed, West Virginia, USA. Sites 3, 4, 6, $10,13,18,19,21,22$ are on the main stem; all other sites are at the mouths of tributaries. 11

Figure 3. Thirty-minute (a) time-series of air temperature (Ta) and Tw during the 2018 annual year and (b) time-series of precipitation during the 2018 annual year collected from a climate station located near site \#13 (Figure 1) in West Run Watershed, West Virginia, USA.

Figure 4. Five-minute water temperature data shown on a (a) site by site, (b) quarterly, and (c) monthly basis collected from 22 gauging sites in West Run Watershed, West Virginia, USA; during the 2018 annual year.

Figure 5. Results of principle component analysis, showing biplots of extracted principle components of annual water temperature data (mean (a), maximum (c), and minimum (e)), and biplots with extracted principle components of quarterly water temperature data (mean (b), maximum (d), and minimum (f)) collected during the 2018 annual year at the 21 monitoring sites of West Run Watershed, WV, USA; and their corresponding LULC area $\left(\mathrm{km}^{2}\right)$. 25 Figure 6. Average annual Tw variables (mean Tw, minimum Tw, and maximum Tw) vs. Cumulative LULC types (forested, agriculture, and mixed development) (1=100\%) moving from the headwaters to the terminus in West Run Watershed, West Virginia, USA, during the 2018 annual year. 29

Figure 7. Stream water temperature surges sensed at sites in West Run Creek following summer precipitation event during the summer of 2018. Black circles mark the peak Tw surges and arrows track the Tw surge as it moves downstream. (a) Stream water temperature surge one occurring on 06/17 at 04:15 (15.1mm precipitation event) moving through West Run Creek. (b) Stream water temperature surge two occurring on 07/25 at 12:00 (13.5mm precipitation event) moving through West Run Creek. (c) Stream water temperature surge three occurring on 07/16 at 13:00 (19.4mm precipitation event) moving through West Run Creek. (d) Stream water temperature surge four (left) occurring on 08/16 at 15:55 (11.7mm precipitation event) and 
stream water temperature surge five (right) occurring on 08/16 at 16:15 (11.7mm precipitation).

Figure 8. Mean (a), standard deviation (b), maximum (c), and minimum (d) water temperature collected at main stem gauging sites of West Run Watershed, West Virginia, USA; plotted against stream distance from headwaters of West Run Creek. Percent mixed development LULC is also plotted against stream distance from headwaters. A reference line for mean Ta over the entire 2018 annual year is included in mean plot (a) ........................................................... 45 Figure A1. Time-series of ambient air temperature (Ta) and average net radiation across the 2018 annual year collected from a climate station located in West Run Watershed, West Virginia, USA. 53

Figure A2. Time-series of relative humidity and precipitation across the 2018 annual year collected from a climate station located in West Run Watershed, West Virginia, USA 53 Figure A3. Time-series of ambient air temperature (Ta) and precipitation across the 2018 annual year collected from a climate station located in West Run Watershed, West Virginia, USA...... 54 Figure B1. Time-series of steam water temperature across the 2018 annual year recorded at gauging site \#1 located in West Run Watershed, West Virginia, USA. 55 Figure B2. Time-series of steam water temperature across the 2018 annual year recorded at gauging site \#2 located in West Run Watershed, West Virginia, USA. 56 Figure B3. Time-series of steam water temperature across the 2018 annual year recorded at gauging site \#3 located in West Run Watershed, West Virginia, USA. 57

Figure B4. Time-series of steam water temperature across the 2018 annual year recorded at gauging site \#4 located in West Run Watershed, West Virginia, USA. 58 Figure B5. Time-series of steam water temperature across the 2018 annual year recorded at gauging site \#5 located in West Run Watershed, West Virginia, USA. 59 Figure B6. Time-series of steam water temperature across the 2018 annual year recorded at gauging site \#6 located in West Run Watershed, West Virginia, USA. 60 Figure B7. Time-series of steam water temperature across the 2018 annual year recorded at gauging site \#7 located in West Run Watershed, West Virginia, USA.

Figure B8. Time-series of steam water temperature across the 2018 annual year recorded at gauging site \#8 located in West Run Watershed, West Virginia, USA. 
Figure B9. Time-series of steam water temperature across the 2018 annual year recorded at gauging site \#9 located in West Run Watershed, West Virginia, USA....

Figure B10. Time-series of steam water temperature across the 2018 annual year recorded at gauging site \#10 located in West Run Watershed, West Virginia, USA. 64

Figure B11. Time-series of steam water temperature across the 2018 annual year recorded at gauging site \#11 located in West Run Watershed, West Virginia, USA.

Figure B12. Time-series of steam water temperature across the 2018 annual year recorded at gauging site \#12 located in West Run Watershed, West Virginia, USA 66 Figure B13. Time-series of steam water temperature across the 2018 annual year recorded at gauging site \#13 located in West Run Watershed, West Virginia, USA

Figure B14. Time-series of steam water temperature across the 2018 annual year recorded at gauging site \#14 located in West Run Watershed, West Virginia, USA.

Figure B15. Time-series of steam water temperature across the 2018 annual year recorded at gauging site \#15 located in West Run Watershed, West Virginia, USA.

Figure B16. Time-series of steam water temperature across the 2018 annual year recorded at gauging site \#16 located in West Run Watershed, West Virginia, USA.

Figure B17. Time-series of steam water temperature across the 2018 annual year recorded at gauging site \#17 located in West Run Watershed, West Virginia, USA.

Figure B18. Time-series of steam water temperature across the 2018 annual year recorded at gauging site \#18 located in West Run Watershed, West Virginia, USA 72

Figure B19. Time-series of steam water temperature across the 2018 annual year recorded at gauging site \#19 located in West Run Watershed, West Virginia, USA.

Figure B20. Time-series of steam water temperature across the 2018 annual year recorded at gauging site \#20 located in West Run Watershed, West Virginia, USA

Figure B21. Time-series of steam water temperature across the 2018 annual year recorded at gauging site \#21 located in West Run Watershed, West Virginia, USA. 75 Figure B22. Time-series of steam water temperature across the 2018 annual year recorded at gauging site \#22 located in West Run Watershed, West Virginia, USA

Figure C1. Results of site by site annual comparison using a Kruskal Wallis ANOVA with a post hoc analysis of mean, maximum, and minimum daily Tw collected at twenty-one sites located in West Run Watershed, West Virginia, USA. Only significant $(\alpha=0.05)$ p-value are shown........ 77 
Figure C2. Results of site by site quarterly and monthly comparison using a Kruskal Wallis ANOVA with a post hoc analysis of mean daily Tw collected at twenty-one sites located in West Run Watershed, West Virginia, USA. Only significant $(\alpha=0.05)$ p-value are shown. (Part 1) .... 78 Figure C3. Results of site by site quarterly and monthly comparison using a Kruskal Wallis ANOVA with a post hoc analysis of maximum daily Tw collected at twenty-one sites located in West Run Watershed, West Virginia, USA. Only significant $(\alpha=0.05)$ p-value are shown. (Part 1)

Figure C4. Results of site by site quarterly and monthly comparison using a Kruskal Wallis ANOVA with a post hoc analysis of minimum daily Tw collected at twenty-one sites located in West Run Watershed, West Virginia, USA. Only significant $(\alpha=0.05)$ p-value are shown. (Part 1)

Figure D1. Results of principle component analysis, showing biplots of extracted principle components of mean monthly water temperature data collected during the 2018 annual year at the 22 monitoring sites of West Run Watershed, WV, USA; and their corresponding LULC area $(\mathrm{km} 2)$

Figure D2. Results of principle component analysis, showing biplots of extracted principle components of maximum monthly water temperature data collected during the 2018 annual year at the 22 monitoring sites of West Run Watershed, WV, USA; and their corresponding LULC area $(\mathrm{km} 2)$

Figure D3. Results of principle component analysis, showing biplots of extracted principle components of minimum monthly water temperature data collected during the 2018 annual year at the 22 monitoring sites of West Run Watershed, WV, USA; and their corresponding LULC area $(\mathrm{km} 2)$ 


\section{LIST OF TABLES}

Table

Page

Table 1. Original LULC classifications from the 2018 NAIP data and the reclassification developed for use when analyzing Tw and LULC relationships in West Run Watershed, West Virginia, USA.

Table 2. Original LULC classifications from the 2018 NAIP data and the reclassification developed for use when analyzing Tw and LULC relationships in West Run Watershed, West Virginia, USA

Table 3. Descriptive statistics of stream water temperature $\left({ }^{\circ} \mathrm{C}\right)$, annually by site, quarterly (all sites), and monthly (all sites) collected in West Run Watershed, West Virginia, USA, over the 2018 annual year.

Table 4. Site by site comparison of annual maximum (parenthetic) daily Tw, along with dominant LULC, at twenty-one sites at West Run Watershed, West Virginia, USA, over the 2018 annual year. Mix Dev represents the LULC type mixed development. Only significant $(\alpha=0.05)$ results from the analysis are shown. 19

Table 5. Spearman correlations between LULC types and maximum Tw of all 21 sites, mainstem, and tributaries of West Run Watershed, West Virginia, USA, during the 2018 annual year. Positive correlations indicate an increase in Tw with increasing LULC and negative correlations indicate a decrease in Tw with increasing LULC. Bold values are significant correlations at $\alpha=0.05 . \mathrm{Q}-$ quarter.

Table 6. Descriptive statistics of stream water temperature surges identified during Quarter 3 of the 2018 annual water temperature data collected at West Run Watershed, West Virginia, USA.

Table E1. Spearman correlations between LULC types and mean, minimum, and standard deviation of Tw at all 21 sites, mainstem, and tributaries of West Run Watershed, West Virginia, USA; during the 2018 annual year. Positive correlations indicate an increase in Tw with increasing LULC and negative correlations indicate a decrease in Tw with increasing LULC. Bold values are significant correlations at $\alpha=0.05$. 


\section{CHAPTER I \\ A SPATIALLY DISTRIBUTED INVESTEGATION OF STREAM WATER TEMPERATURE IN A CONTEMPORARY MIXED-LAND-USE WATERSHED}

\section{Background}

Stream water temperature (Tw) affects abiotic and biotic processes in aquatic ecosystems (Caissie, 2006; Webb et al., 2008). Abiotic variables influenced by Tw include dissolved oxygen concentration, chemical reaction rates, viscosity, density, and surface tension (Webb, 1996). Biological processes influenced by Tw include the growth rate of fish (Coutant, 1999) and rates of primary production in some autotrophic species (Winder and Sommer, 2012). Water temperature therefore impacts multiple trophic levels of the aquatic food web, including periphyton, benthic macroinvertebrates, and fishes (Pollock et al., 2009). This is of further relevance given that many aquatic organisms (e.g., mottled sculpin (Cottus bairdii), Escherichia coli, flathead mayfly (Heptageniidae sp)) have specific tolerance ranges for Tw (Coutant, 1999). Water temperature tolerances/preferences also directly affect species distributions and population densities, thus affecting important industries (i.e. fishing) (Martin and Petty, 2009; Petty et al., 2014; Petty et al., 2012). Globally, water temperature has traditionally received less attention relative to other water quality parameters, such as suspended sediment and water chemistry (Webb et al., 2008). However, the ecohydrological importance of Tw and susceptibility to anthropogenic disturbance make it a critical variable of concern for resource managers (Coutant, 1999; Poole and Berman, 2001).

The landscape of most contemporary watersheds consists of many different land-use landcover types (LULC) that influence air temperature, rainfall-runoff temperatures, and therefore receiving water temperature (Borman and Larson, 2003; Kellner et al., 2018; Mustafa et al., 2018). These LULC types also influence groundwater surface water interactions influencing Tw (Story et al., 2003). Many studies have shown that incident shortwave radiation is the variable of greatest influence on stream Tw (Gravelle and Link, 2007; Moore et al., 2006; Pollock et al., 2009). Vegetation intercepts incoming shortwave radiation, reducing the amount of radiation reaching the surface thereby reducing surface temperatures (Moore et al., 2006; Oke, 1987; Pollock et al., 2009). For example, Webb and Zhang $(1997,1999)$ showed that incident shortwave radiation accounted for $70 \%$ of a stream's thermal inputs, whereas other significant sources of energy come from longwave radiation emitted by atmospheric water vapor, and advected energy from the 
stream bed and bank. Inter-catchment variations in aspect, elevation, and catchment size have also been shown to differentially affect Tw (Webb and Nobilis, 1997).

\section{Ta-Tw Relationship}

Ambient air temperature (Ta) has been shown to have a substantial influence on $\mathrm{Tw}$ (O’Driscoll and DeWalle, 2006; Rice et al., 2011; Sinokrot and Stefan, 1993). This is important because the Ta and Tw relationship could mask impacts of land-use on Tw. Research has shown conclusively that, as water moves downstream, it is impacted less by the various inflows from runoff/tributaries, and more so by ambient air temperature (Ta) (O'Driscoll and DeWalle, 2006; Sinokrot and Stefan, 1993). However, it should also be stated that land-use also influences Ta in addition to Tw (Moore et al., 2005). Rice et al. (2011) showed that increasing watershed urbanization causes the Tw and Ta relationship to break down. Tw begins to deviate from Ta as energy and mass accumulate from surrounding mixed development LULC types. This is, in some instances, offset given that higher channel volumes downstream are often sufficient enough to attenuate thermal inputs (Caissie, 2006; Gu et al., 1999). Thus, stream inputs (e.g., groundwater, surface runoff, tributaries) from surrounding land-use types may have a greater influence upstream near headwaters relative to downstream and during periods of low(er) flow relative to periods of flooding (Webb et al. 2008). Ultimately, water volume and Ta are not the only influencing factors affecting Tw, and the impacts of complex LULC must be considered (Moore et al., 2006; Pollock et al., 2009; Rice et al., 2011; Younus et al., 2000; Zeiger and Hubbart, 2015).

\section{Groundwater Tw Interactions}

Surface-subsurface exchanges through groundwater inputs can contribute to a waterbody's thermal regime (Marzadri et al., 2013; Webb et al., 2008). Story et al. (2003), observed that groundwater inflows into a stream were responsible for approximately $40 \%$ of the gross cooling of Tw when passing from open to shaded regions. Researchers often assume that groundwater influences Tw in smaller first order tributaries more so than the mainstem. These groundwater inputs can lower Tw variability of smaller-scale streams at both a daily and seasonal scale (Marzadri et al., 2013; Webb et al., 2008). The lowering of stream Tw variability in these cases typically comes from the groundwater's constant temperature compared to the variable surface Tw (Marzadri et al., 2013). However, this is not always the case and groundwater inputs into smaller scale streams can increase Tw variability if groundwater 
temperatures are drastically different from overlying Tw (Anderson et al., 2010). This increase in Tw variability due to groundwater inflow can vary on both a seasonal and diel scale (O'Driscoll and DeWalle, 2006). For example, modeling research by Herb and Stefan (2011) in a Minnesota cold water stream observed that groundwater input increased daily and monthly Tw variability. Groundwater temperature variability also depends on characteristics of the underlying aquifer (e.g., aquifer material, confined vs unconfined). Similar to surface waters aquifers often display seasonal temperature variations (Marzadri et al., 2013).

\section{Forested LULC Types}

Forested land use is commonly associated with lower Tw fluctuations and lower overall Tw, particularly in warmer months (Moore et al., 2005). Increases in stream temperature are commonly observed subsequent to the removal of riparian buffers or riparian zone (RZ) (Bulliner and Hubbart, 2013; Gravelle and Link, 2007; Imhoff et al., 2010; Johnson and Almlöf, 2016; Johnson and Jones, 2011; Moore et al., 2005). This finding was attributed primarily to the function of vegetative stream buffers to attenuate thermal inputs through interception of incoming shortwave radiation (Gregory et al., 1991; Naiman and Décamps, 1997). Although interception of incoming shortwave radiation by the forest canopy is the primary regulating variable influencing Tw in forested LULCs, it is not the sole influencing variable (Moore et al., 2005). Energy exchanges set by the RZ boundary conditions influence Tw, and at the same time, Tw is one of the boundary conditions influencing riparian microclimates boundary conditions (Moore et al., 2005). Forest canopies influence the thermal and moisture conditions below canopy by not only reducing solar radiation but also intercepting precipitation, reducing wind speeds, and increasing longwave radiation to the forest floor (Moore et al., 2005; Oke, 1987; Xuhui, 2018). Typically forest canopies reduce the diel fluctuation of ambient air temperatures (Ta) compared to open areas associated with some agricultural and mixed development LULCs (Johnson and Jones, 2011; Moore et al., 2005; Oke, 1987; Pollock et al., 2009; Xuhui, 2018). The canopy can also influence conditions such as relative humidity and vapor pressure deficits (Moore et al., 2005). For example, during a summer day, relative humidity tends to be 5 to 25 percent higher in the forest because of the homogenous vapor pressure within an air mass and the lower daytime temperatures under forest canopies (Moore et al., 2005).

\section{Agricultural LULC Types}


Agricultural land-use types are typically associated with higher Tw (Younus et al., 2000; Horne and Hubbart, 2020). This may be due to turbid waters, subsurface lateral flow and increased shortwave radiation. For example, turbid water associated with agricultural land-use practices has been shown to have higher concentrations of total suspended solids (TSS) and, thus, higher Tw relative to clear water due to particulate matter heat adsorption (Boyde et al., 2016; Oke, 1987). Younus et al. (2000) created a numerical model to compute free surface flow hydrodynamics and coupled stream temperature dynamics in an agricultural watershed. Model results showed that an increase in subsurface lateral flow and shortwave radiation were the most significant contributors to the stream heat budget (Younus et al., 2000). Interestingly, the influence of subsurface flow on the stream heat budget was comparable to shortwave radiation (Younus et al., 2000). This strong influence by subsurface flow is attributable to water being heated while infiltrating and percolating through the surface soil during periods of bare fields and high quarterly radiation. Oke (1987) noted that crops can provide some shade through increased shortwave interception and reduce runoff volumes through transpiration. This is as contrast with the period after harvest when runoff volumes are higher and the soil is exposed to greater amounts of shortwave radiation causing soils to reach higher temperatures (Campbell and Norman, 1998; Hatfield et al., 2001; Oke, 1987; Younus et al., 2000). Similar heating processes, associated with runoff, also occur in mixed development LULC types (Kinouchi et al., 2007; Palmer and Nelson, 2007; Zeiger and Hubbart, 2015).

\section{Mixed Development LULC Types}

Previous research showed that mixed development LULC types increase the volume of heated runoff entering adjacent streams during summer precipitation events (Herb et al., 2008; Rice et al., 2011; Zeiger and Hubbart, 2015). This has been attributed to stormwater runoff contacting heated impervious surfaces (Herb et al., 2008; Palmer and Nelson, 2007; Paul and Meyer, 2001; Zeiger and Hubbart, 2015). As heated runoff enters adjacent water bodies, it can result in a thermal surge (Herb et al., 2008; Rice et al., 2011; Zeiger and Hubbart, 2015). Thermal surges were characterized as a $1^{\circ} \mathrm{C}$ increase in Tw within fifteen minutes following a rain event (Rice et al., 2011; Zeiger and Hubbart, 2015). For example, Rice et al. (2011) supported this finding, showing a linear relationship between percent impervious surface and mean thermal surge amplitude, where surges of $3.3^{\circ} \mathrm{C}$ were observed in areas with $75 \%$ impervious surface cover. Zeiger and Hubbart (2015) observed surge increases of $4^{\circ} \mathrm{C}$ lasting for up to five hours in work conducted in the central United 
States. Thermal point source inputs in mixed development land-use types, particularly those from stormwater or wastewater outlets, can also impact Tw. Kinouchi et al. (2007) showed a positive correlation between increasing annual $\mathrm{Tw}$ and an increase in volume of heated wastewater input from wastewater treatment plants. This increase was $0.1-0.2^{\circ} \mathrm{C} /$ year resulting in an overall increase in $4.2^{\circ} \mathrm{C}$ over twenty years. Other research showed that mixed-development (urban) areas can increase subsurface temperatures through above ground (urban heat island effect) to below ground advection (Menberg et al., 2013; Oke, 1987; Zeiger and Hubbart, 2015).

\section{Tw Research in Appalachia}

The Appalachian region of the United States is an example of a physiographically complex region with high relief relative to surrounding areas (Raitz, 2019). Factors such as higher relief alter the rate of runoff from surrounding land-use types relative to flatter terrain (Zajiček et al., 2011). Although previous Tw studies have been conducted in other physiographically complex regions, such as the Rocky Mountains of the United States, the Appalachian region differs due to being geologically older and more weathered (Blackwelder, 1914). Thus, a region such as Appalachia is well suited to advance existing knowledge gaps in the relationship between landuse types and Tw. Furthermore, in recent years Appalachia, in particular the state of West Virginia, has been impacted by increased flood frequency largely attributed to an increase in percent mixed development land-use type. As mixed development land-use types expand they often replace forested land-use types (LeBlanc et al., 1997). This is important because previous research showed these two land-use classifications have distinct effects on Tw (Webb et al. 2008). Furthermore, the interactions between competing mixed land-use types are largely unknown. Thus, studies in a contemporary mixed-land-use watershed designed to assess land-use impacts on hydrologic variables, including Tw, are needed in general (globally) and in the region (specifically) (Hubbart et al. 2019).

\section{The Nested Experimental Watershed Study Design}

Using the experimental watershed study design in a contemporary mixed-land-use watershed, researchers can separate a larger catchment in to individual sub-catchments, enabling quantification of specific land use impacts on variables of interest (Hewlett et al., 1969; Hubbart et al., 2019; Kellner et al., 2018; Kellner and Hubbart, 2017a; Tetzlaff et al., 2017). The paired and nested-scale experimental watershed study design approach was initially developed by forestry 
researchers analyzing the effects of silvicultural practices and road building on stream hydrology and water quality (Gravelle and Link, 2007; Hubbart et al., 2007). The approach is used much less frequently than other methodology. This is primarily attributed to the noted high cost of instrumentation, often the time-consuming nature of data collection, difficulty in transferring results, and unrepresentativeness as being prohibitive to implementation (Hewlett et al., 1969; Kellner and Hubbart, 2017a; Tetzlaff et al., 2017). Although some of these perceived problems are not unfounded, the inherent advantages of an experimental watershed study design far outweigh the potential disadvantages (Hewlett et al., 1969; Kellner and Hubbart, 2017a; Tetzlaff et al., 2017). The experimental watershed study design has been shown to effectively characterize and quantify both hydrologic and water quality perturbation (Hewlett et al., 1969; Hubbart et al., 2019; Tetzlaff et al., 2017). It also is effective at addressing both site-specific management questions and assist further model development, validation, and calibration (Hewlett et al., 1969; Hubbart et al., 2019; Tetzlaff et al., 2017).

\section{Statement of Need}

There is an ongoing need for high-resolution studies in contemporary watersheds that include multiple land use practices (Hubbart et al., 2019; Petersen and Hubbart, 2020; Zeiger et al., 2016). Unlike West Run Watershed, other study watersheds in the Appalachian region, such as those in the Fernow Experimental Forest, are less instrumented and comprise homogenous land use. In addition, there is a lack of watershed-scale studies that utilize the experimental watershed study design in Appalachia, or elsewhere. The use of high-resolution observed data provides a greatly needed investigation increasing confidence in underlying processes, both physical and chemical, occurring on the landscape (Kellner et al., 2018; New et al., 2002). At the time of this research, no previous studies gathering high-resolution Tw have been conducted in the Appalachian region of the United States using an experimental watershed study design. The lack of such studies may be in part due to the experimental watershed study design's criticisms (Hewlett et al., 1969; Hubbart et al., 2019; Tetzlaff et al., 2017). However, such studies will advance spatial and temporal understanding and therefore improve management decisions in contemporary (municipal) watersheds (Kellner et al., 2018; New et al., 2002). 


\section{Objectives}

The overall objective of this study was to use an experimental watershed study design to investigate land-use practices impacts on Tw, particularly maximum Tw, spatially and temporally in a representative contemporary mixed-land-use watershed. Specific objectives included quantifying, a) annual, b) quarterly, and c) monthly relationships between LULC and Tw.

\section{Hypotheses}

1.) $\mathrm{H}_{0}$ : Land-use practices in West Run Watershed will have no significant effect on stream Tw at each timescale $\mathrm{H}_{\mathrm{a}}$ : Land-use practices in West Run Watershed will have significant effects on stream Tw at each timescale

2.) $\mathrm{H}_{0}$ : There will not be a significant relationship between LULC and Tw between quarters and months.

$\mathrm{H}_{\mathrm{a}}$ : There will be a significant relationship between LULC and Tw between quarters and months

3.) $\mathrm{H}_{\mathrm{a}}$ : There will be a significant relationship between LULC and Tw between quarters and months. $\mathrm{H}_{0}$ : There will not be a significant difference in daily maximum Tw across different sites between quarters and months. $\mathrm{H}_{\mathrm{a}}$ : There will be a significant difference in daily maximum Tw across different sites between quarters and months

\section{Materials and Methods}

\section{Study Site}

West Run Watershed (WRW) is located in the Monongahela Watershed and categorized as a Hydrologic Group D watershed (HUC \#05020003) located near Morgantown, West Virginia, USA. The watershed spans $23 \mathrm{~km}^{2}$, and the main drainage, West Run Creek, drains directly into the Monongahela River. The channel of West Run, according to habitat surveys, lacks sinuosity in many reaches and possesses a low channel slope of $1.1 \%$, with back watering (flooding) near the terminus (WVWRI, 2008). Average net radiation in Morgantown from 1981-2010 was $130.68 \mathrm{~W} / \mathrm{m}^{2}$ (Arguez 
et al. 2012). The average recorded precipitation depth in Morgantown is approximately $1096 \mathrm{~mm} / \mathrm{yr}$ (1981-2010). Precipitation falls throughout the year but increases in quantity during the spring and summer months (Kutta and Hubbart, 2019a). Previous studies showed that precipitation has significantly ( $\mathrm{p}=0.01$ ) increased in the Appalachian region by $2.2 \%$ over the past 111 years (Kutta and Hubbart, 2019a; Petersen et al., 2018). Precipitation is frequently generated via frontal storm convergence systems, but during the summer months in particular, precipitation also occurs via orographic and / or convective processes (Kutta and Hubbart, 2019b).

The current population of Morgantown is 30,539. Overall land-use in West Run Watershed is $19.4 \%$ agriculture, $42.7 \%$ forest, and $37.7 \%$ mixed development, with the portion of agricultural land-use mainly comprising animal husbandry (i.e., cattle), and crop fields (i.e., corn, soybeans, cover crops) (Kellner et al., 2018; Petersen et al., 2018). The presence of different land-use types, coupled with regular land-use manipulation via human development, justifies WRW categorization as a representative contemporary mixed-land-use watershed (Kellner et al., 2018). In addition, a combination of agricultural, mining, and industrial land-uses in and surrounding WRW have contributed to ongoing land and water resource degradation (Kellner et al., 2018). Given the potential for legacy effects of historic land-use land practices including, but not limited to, historical mining operations, those practices need to be chronicled and included in analyses of work (Kellner and Hubbart, 2017b; Petersen and Hubbart, 2020). West Run Watershed has a history of surface and subsurface coal mining, particularly in the headwaters of the watershed, and peaking in the 1930s, 40s, and 50s (West Virginia Water Research Institute, 2008). Mining practices ceased by the late 1950s, however, evidence of those operations remains apparent by means of acid mine drainage (AMD). During the 1940s, the population increased by 8,870 from approximately 16,000 individuals at an annual growth rate of 4.36\% per year (United States Census Bureau, 2020). During the 1940s, mixed development areas such as the Walter L. Bill Hart Field (i.e., Morgantown Municipal Airport) were constructed in West Run (West Virginia Department of Transpiration, 2008). By the late 1950's, population in Morgantown decreased by 3030 as mining activities decreased. Population surged in the decade to follow (i.e., the 1960's) reaching 29,431 (United States Census Bureau, 2020). Since the 1960s, the population has remained at approximately 30,000 individuals. However, since the 1970s, the LULC composition of West Run Watershed has continued to change. 
Comparing Historical LULC data from 1992, 2011, to the most recent LULC data (2018), mixed development areas increased from $18.2 \%$ in 1992 to $37.8 \%$ in 2018, replacing forested $(54.3 \%$ in 1992 to $42.8 \%$ in 2018) and agricultural (27.4\% in 1992 to $19.2 \%$ in 2018) LULC (WVGIS Tech Center, 2020). Anecdotally observed by the project managers of the watershed-based plan for West Run of the Monongahela River, the increase in urban sprawl stemming from the surrounding city of Morgantown continues to increase the severity and frequency of flooding (WVWRI, 2008).

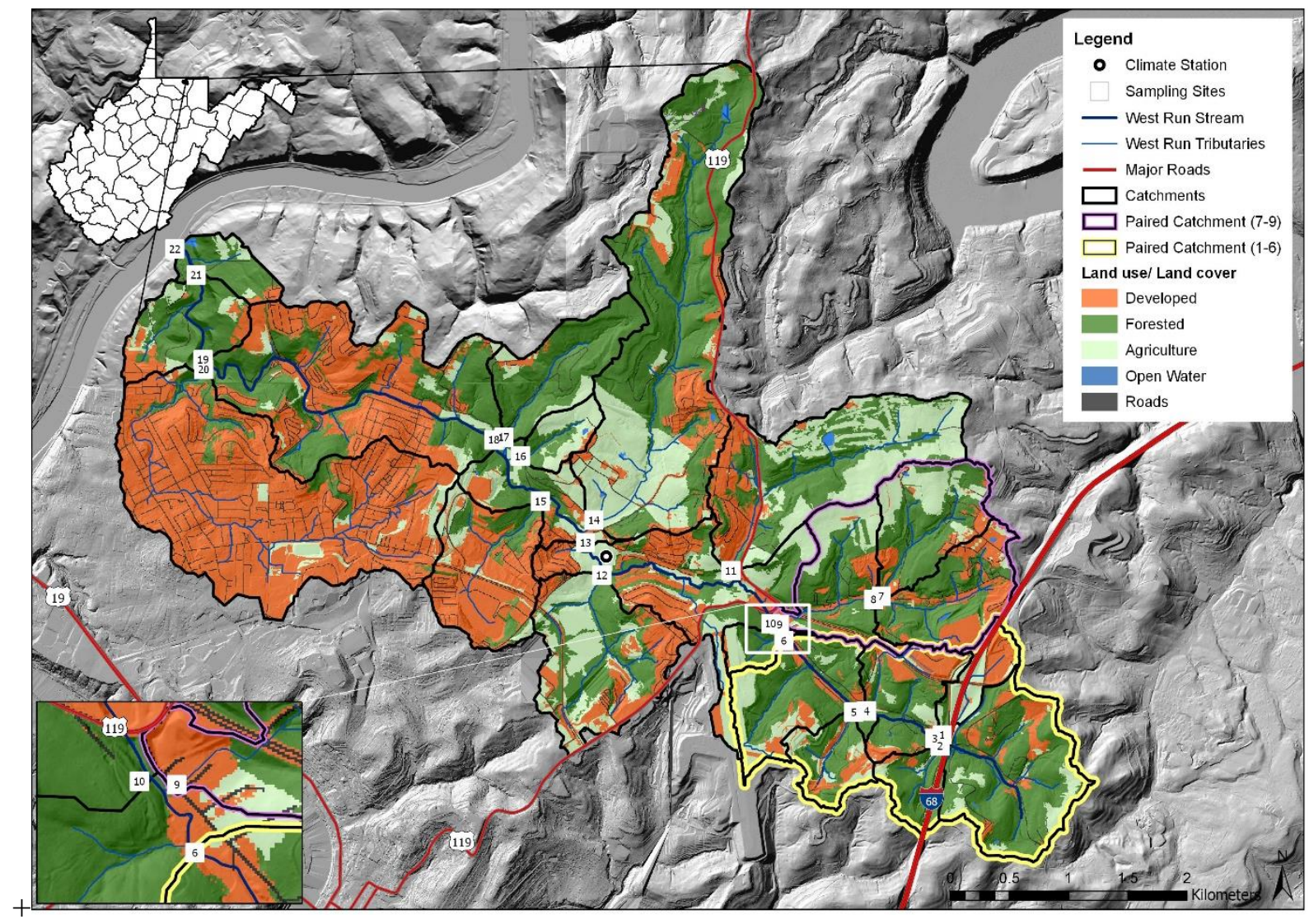

Figure 1. Land use and land cover of West Run Watershed (WRW), West Virginia, USA including 22 nested gauging sites and corresponding sub-basins.

\section{Climate Data}

During the study period, climate data were recorded at 30-minute time intervals using researchgrade climate instrumentation located within approximately $100 \mathrm{~m}$ of Site \#13 (Figure 1). Climate 
variables (recorded at a height of $3 \mathrm{~m}$ ) included precipitation (TE525 Tipping Bucket Rain Gauge), average air temperature and relative humidity (Campbell Scientific HC2S3 Temperature and Relative Humidity Probe), average wind speed (Met One 034B Wind Set instrument), and net radiation (Campbell Scientific NR01 Four-Component Net Radiation Sensor).

\section{LULC Data}

LULC was derived from National Agricultural Imaging Program (NAIP) 2018 data. Initially, LULC types included 16 different assignations. For the current work, each of the original 16 LULC types was further grouped (lumped) into one of four LULC categories MixedDeveloped, Agriculture, Forested, and Open water (Table 1). Forested land-use practices are the most abundant land use classification in the WRW. For the current work, land-use land-cover types grouped in the Forested category included mature vegetation and also mine grass classification, which is assumed to have succeeded to an intermediate successional stage (Grime, 1998, 1973). This assumption was validated through onsite inspection of these areas. Agricultural land-use practices were those classifications associated with early successional stages (i.e., low vegetation) or pastures and fields. Mixed development land-use practices included mixed development and all other LULC classifications associated with urban areas and/or impervious surfaces (e.g. barren, roads, impervious) (Table 2) (Booth et al., 2016; Paul and Meyer, 2001; Rice et al., 2011; Walsh et al., 2005). Using Arc GIS, watershed and sub-catchment boundaries were delineated, and LULC data incorporated. Each pixel representing $5 \mathrm{~m}^{2}$ was counted and converted to $\mathrm{km}^{2}$ and used to estimate percent LULC for each subcatchment draining to each monitoring site (Table 2 and Figure 2). 
Table 1. Original LULC classifications from the 2018 NAIP data and the reclassification developed for use when analyzing Tw and LULC relationships in West Run Watershed, West Virginia, USA.

\begin{tabular}{cc}
\hline Reclassified & Original LULC Classification \\
\hline Mixed Development & Roads, Impervious, Mixed development, Barren \\
Agriculture & Low vegetation, Hay pasture, Cultivated crops \\
& Mine grass, Forest, Mixed mesophytic forest, Dry \\
Forested & $\begin{array}{c}\text { mesic oak forest, Dry oak forest, Small stream } \\
\text { riparian habitats }\end{array}$ \\
Open water & Water, River floodplains, and Palustrine Emergent \\
& Wetlands \\
\hline
\end{tabular}
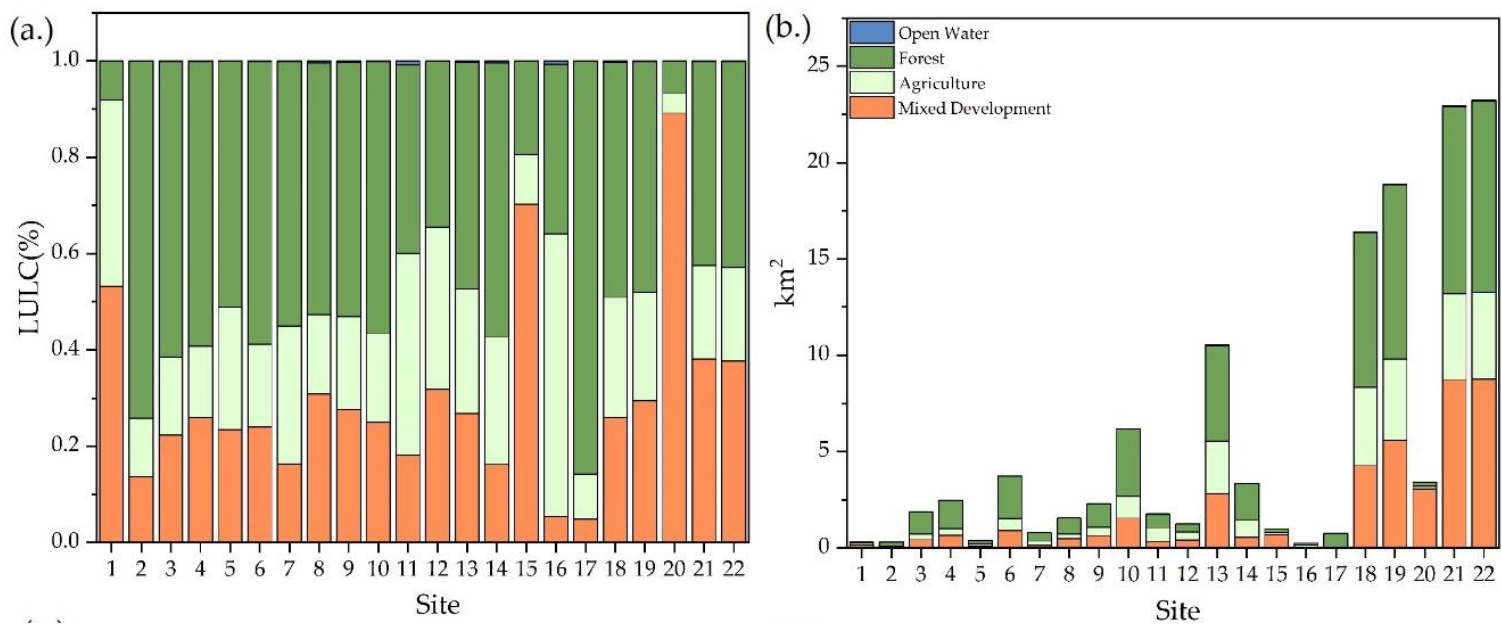

Figure 2. (a) Proportion LULC of sub-catchment and associated sites, (b) LULC area of subcatchment and each associated site in West Run Watershed, West Virginia, USA. Sites 3, 4, 6, $10,13,18,19,21,22$ are on the main stem; all other sites are at the mouths of tributaries. 
Table 2. Sub-basin area $\left(\mathrm{km}^{2}\right)$ and proportional contribution to West Run Watershed (\%, in parenthesis), West Virginia, USA from NAIP (2018). Area $\left(\mathrm{km}^{2}\right)$ and percent of sub-basin (\%, in parenthesis) are given for each LULC type for each site. Site 22 LULC areas and proportions represent WRW as a whole. Cumulative open water $\left(0.295 \mathrm{~km}^{2}\right)$ was excluded.

\begin{tabular}{ccccc}
\hline & & \multicolumn{3}{c}{ Land Use / Land Cover } \\
Site & Sub-basin Area & Mixed Development & Agriculture & Forested \\
\hline 1 & $0.3(1.3)$ & $0.2(53.2)$ & $0.1(38.7)$ & $0.0(8.1)$ \\
2 & $0.3(1.3)$ & $0.0(13.6)$ & $0.0(12.2)$ & $0.2(74.2)$ \\
3 & $1.9(8.0)$ & $0.4(22.4)$ & $0.3(16.7)$ & $1.1(61.3)$ \\
4 & $2.5(10.7)$ & $0.6(25.9)$ & $0.4(14.9)$ & $1.5(59)$ \\
5 & $0.4(1.6)$ & $0.1(23.4)$ & $0.1(25.5)$ & $0.2(51.1)$ \\
6 & $3.7(16.0)$ & $0.9(23.9)$ & $0.6(17.2)$ & $2.2(58.7)$ \\
7 & $0.8(3.4)$ & $0.1(16.3)$ & $0.2(28.6)$ & $0.4(54.9)$ \\
8 & $1.6(6.7)$ & $0.5(30.8)$ & $0.3(16.5)$ & $0.8(52.4)$ \\
9 & $2.3(9.8)$ & $0.6(23.9)$ & $0.4(19.3)$ & $1.2(52.8)$ \\
10 & $6.2(26.6)$ & $1.5(24.9)$ & $1.1(18.4)$ & $3.5(56.5)$ \\
11 & $1.8(7.5)$ & $0.3(18.2)$ & $0.7(41.9)$ & $0.7(39.2)$ \\
12 & $1.2(7.5)$ & $0.4(31.8)$ & $0.4(33.7)$ & $0.4(34.5)$ \\
13 & $10.5(45.3)$ & $2.8(26.8)$ & $2.7(25.8)$ & $5.0(47.1)$ \\
14 & $3.4(14.4)$ & $0.5(16.2)$ & $0.9(26.4)$ & $1.9(56.9)$ \\
15 & $1.0(4.2)$ & $0.7(70.3)$ & $0.1(10.3)$ & $0.2(19.4)$ \\
16 & $0.2(1.1)$ & $0.0(5.4)$ & $0.1(58.7)$ & $0.1(35.2)$ \\
17 & $0.7(3.2)$ & $0.0(4.8)$ & $0.1(9.4)$ & $0.6(85.8)$ \\
18 & $16.4(70.6)$ & $4.3(26.0)$ & $4.1(24.9)$ & $8.0(48.9)$ \\
19 & $18.9(81.2)$ & $5.6(29.4)$ & $4.2(22.5)$ & $9.0(47.9)$ \\
20 & $3.4(14.7)$ & $3.0(89.2)$ & $0.1(4.2)$ & $0.2(6.6)$ \\
21 & $22.9(98.7)$ & $8.7(38.1)$ & $4.5(19.5)$ & $9.7(42.2)$ \\
22 & $23.2(100.0)$ & $8.8(37.7)$ & $4.5(19.4)$ & $9.9(42.7)$ \\
\hline & & & &
\end{tabular}

Data Analysis

At each of the twenty-two stream monitoring sites a Solinst Levelogger Gold pressure transducer was installed between the fall of 2016 and fall of 2017. Each site was equipped with a Solinst Levelogger Gold pressure transducer that logged and stored Tw $\left({ }^{\circ} \mathrm{C}\right)$ data, with an accuracy of $\pm 0.05^{\circ} \mathrm{C}$, and stage (water depth, $\mathrm{cm}$ ), with an accuracy of $\pm 0.3 \mathrm{~cm}$, at five-minute intervals Data analyses included descriptive statistics (5 min data) of annual, quarterly, and monthly Tw data over the 2018 annual year for each site except for Site 22 due to potential backwatering with the Monongahela River. Quarterly time steps were delineated as; January $1^{\text {st }}-$ March $31^{\text {st }}$ (Quarter 1), April $1^{\text {st }}-$ June 30 ${ }^{\text {th }}$ (Quarter 2), July $1^{\text {st }}-$ September 30 ${ }^{\text {th }}$ (Quarter 3), 
and October $1^{\text {st }}-$ December $31^{\text {st }}$ (Quarter 4). Data post processing included estimation of erroneous data or missing points $(<0.2 \%$ of total data) by averaging between data points on either side of a gap, or by linear interpolation (Helsel and Hirsch, 1992).

The Tw data were shown to be non-normally distributed using the Anderson-Darling test (Kellner et al., 2018). Therefore, correlations between land use and Tw variables (i.e., mean Tw, maximum Tw, minimum Tw, and Tw standard deviation) were determined (Appendix E) using the Spearman Rank correlation coefficient test $(\alpha=0.05)$ generating Spearman's Rank Correlation Coefficients (rs). The open water land-use type was excluded from analyses due to its negligible coverage relative to other land use types in the watershed. Three separate analyses were run including a) all sites b) tributaries only (i.e., sites 1, 2, 5, 7, 8, 9, 11, 12, 14, 15, 16, 17, 20), and c) mainstem West Run Creek only (i.e., sites 3, 4, 6, 10, 13, 18, 21) to assess effects of surrounding land-use practices on tributaries vs. mainstem sites.

Due to the detrimental influence maximum Tw can potentially have on aquatic biological/geochemical processes, a comparison of daily maximum Tw was conducted on a site by site basis using a Kruskal Wallis ANOVA with a post hoc analysis (Boyde et al., 2016; Caissie, 2006). The independent variable in this Kruskal Wallis ANOVA was Site and the dependent variable the daily maximum Tw. The Kruskal Wallis ANOVA was run for each separate time step (i.e., annual, quarterly, monthly). The post hoc analysis consisted of multiple Mann-Whitney $U$ test comparing individual sites on a site by site basis (Helsel and Hirsch, 2020). To control for potential Type I errors while executing the multiple comparisons, a DunnBonferroni correction was implemented (Helsel and Hirsch, 2020).

Multiple Principal Component Analyses (PCA) were conducted to illustrate the relationship between LULC types and Tw variables at an annual, quarterly, and monthly timescale, again excluding open water (Bro and Smilde, 2014). Using OriginPro Pro 9b Academic (OriginLab Corporation), correlation biplots were generated. Due to the use of observed Tw data, no autoscaling preprocessing was needed to compare Tw and LULC data in the PCA analysis. However due to the use of differing units (i.e., proportions (\%) and temperature $\left({ }^{\circ} \mathrm{C}\right)$ ) a correlation matrix was used rather than a covariate matrix (Bro and Smilde, 2014). Following the Kaiser Guttman criterion, Eigenvalues greater than one were accepted as principle components above the threshold of importance (Bro and Smilde, 2014). 
Finally, a preliminary investigation of LULC thresholds (i.e., tipping points) (Lenton, 2013) was conducted to elucidate potential tipping points in WRW. This investigation compared cumulative LULC proportion from headwaters to terminus, and Tw for each of Tw variables, also cumulative, during the 2018 annual year. Cumulative Tw variables (plotted as y-axis 1) were calculated by adding mean, maximum, or minimum Tw at each site, progressing numerically from the headwater to the terminus. Cumulative LULC proportion (plotted as y-axis 2) were calculated by adding successive LULC proportions (0-1) at each site, progressing numerically from the headwater to the terminus. Cumulative LULC proportions can exceed $100 \%(100 \%=1)$ with the addition of successive sites. Both y-axes use logarithmic scaling and the $\mathrm{x}$-axis, as stated, displays the numeric progression of sites (1-21). Points of intersection between cumulative LULC and Tw variables may elude to potential tipping point. If the analysis hints towards the existence of tipping points, these data could potentially be valuable information used to guide land management decisions or policies regarding LULC-Tw relations. 


\section{Results}

\section{Climate during Study}

Total precipitation recorded in West Run Watershed over the 2018 annual year was 1378 $\mathrm{mm}$, which was $282 \mathrm{~mm}$ higher than average annual precipitation (1096 $\mathrm{mm}$ ) over the previous 111 years (Arguez et al., 2012; Petersen et al., 2018). The largest precipitation event observed in a 30-min window was $22.9 \mathrm{~mm}$, occurring on September 9 (Figure 3b). The largest continuous precipitation event $(83.2 \mathrm{~mm})$ began on September $8^{\text {th }}$ at 18:00 and lasted until September 9 at 16:30 (Figure 3b). Mean air temperature (Ta) during the 2018 annual year was $11.6^{\circ} \mathrm{C}$, which was $0.2^{\circ} \mathrm{C}$ higher than the average annual temperature $\left(11.4^{\circ} \mathrm{C}\right)$ in West Virginia between 1990 2016 (Kutta and Hubbart, 2019a). The coldest $\left(-24.8^{\circ} \mathrm{C}\right)$ and warmest $\left(34.6^{\circ} \mathrm{C}\right)$ recorded air temperature occurred January 1 at 8:00 and July 1 at 17:00, respectively (Figure 3a). Maximum net radiation was $1100 \mathrm{~W} / \mathrm{m}^{2}$ recorded on May 7 at 12:00. Mean near surface $(1.5 \mathrm{~m})$ net radiation was $139.5 \mathrm{~W} / \mathrm{m}^{2}$, which was $8.82 \mathrm{~W} / \mathrm{m}^{2}$ higher than the mean annual net radiation in Morgantown between 1981 and 2010 (130.68 W/m²) (Appendix A) (Arguez et al., 2012). 

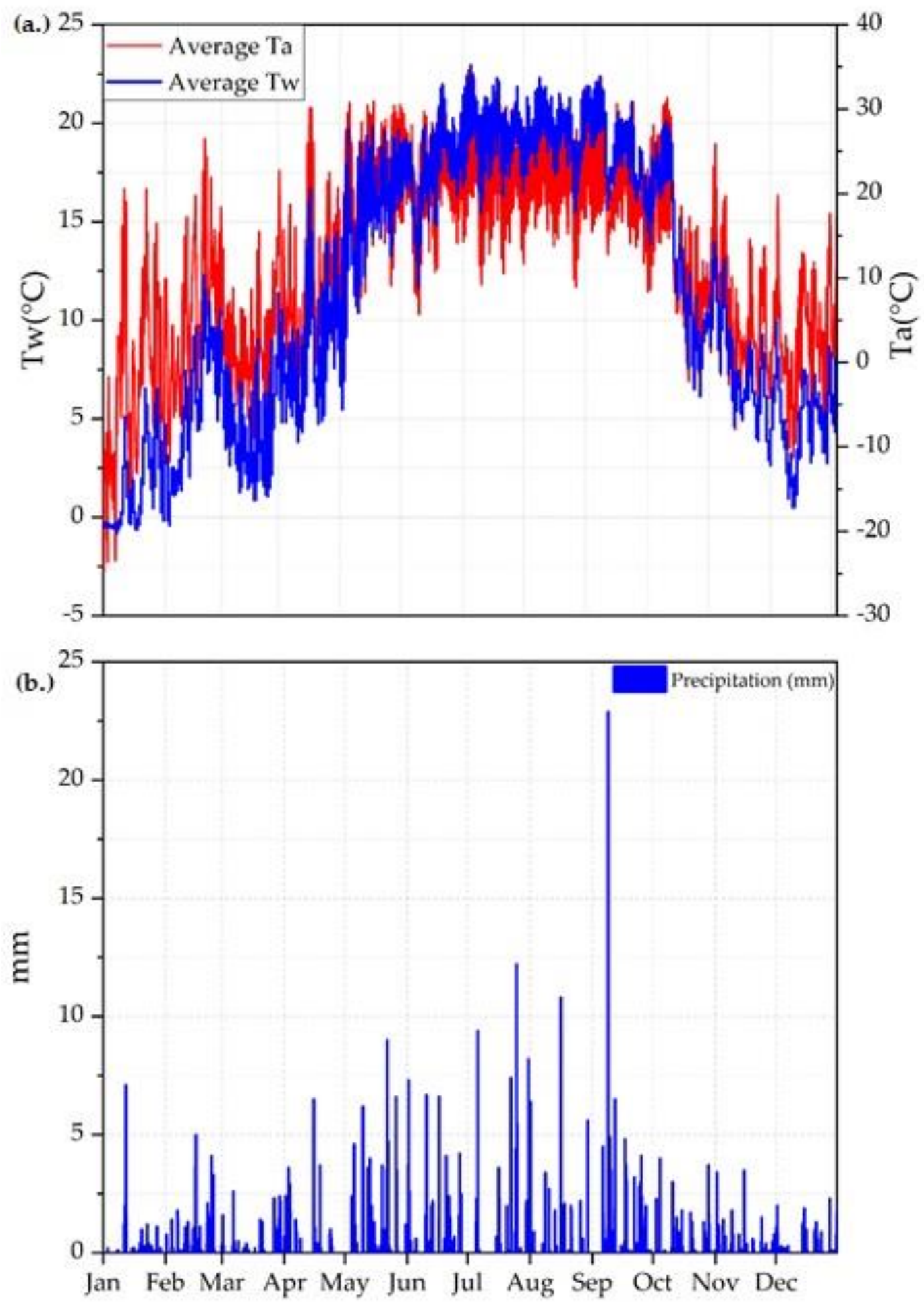

Figure 3. Thirty-minute (a) time-series of air temperature (Ta) and Tw during the 2018 annual year and (b) time-series of precipitation during the 2018 annual year collected from a climate station located near site \#13 (Figure 1) in West Run Watershed, West Virginia, USA. 


\section{Annual Stream Water Temperature}

Annual average water temperature (Tw) across all sites varied by $2.4{ }^{\circ} \mathrm{C}$ with a maximum of $12.5^{\circ} \mathrm{C}$ at site \#11 (dominant LULC Agriculture; $41.9 \%$ ) and a minimum of $10.1{ }^{\circ} \mathrm{C}$ at site \#17 (dominant LULC Forested; 85.8\%) (Table 3). The lowest recorded Tw $\left(-3.0{ }^{\circ} \mathrm{C}\right)$ occurred at Site \#16, (dominant LULC Agriculture; 58.7\%), at 6:45 on January 7, and the highest recorded Tw $\left(27.4{ }^{\circ} \mathrm{C}\right.$ ) occurred at Site \#5, (dominant LULC Forested; 51.1\%) at 16:05 on July 3. Site \#5 includes the Morgantown Municipal Airport, one of the largest expanses of impervious surface covers in the watershed. Both were recorded less than a week after the lowest $\left(-24.8^{\circ} \mathrm{C}\right)$ and highest recorded Ta $\left(34.6{ }^{\circ} \mathrm{C}\right)$. A timeseries for all 22 sites is included in Appendix B.

Spearman correlations of all twenty-one sites across the entire annual year indicated a significant $(\mathrm{p}=0.01)$ positive correlation $(\mathrm{rs}=0.6)$ between maximum Tw and Agriculture LULC area, and a negative correlation $(\mathrm{rs}=-0.5)$ between maximum Tw and Forest LULCs $(\mathrm{p}=0.03)$.

Site by site comparisons indicated that maximum Tw at site \#1 was significantly different from site \#5, \#11, \#12, and \#15 (Table 4) (Appendix C). Maximum Tw at site \#2 was significantly different from sites \#14, \#15, \#18, \#19, \#21. Maximum Tw at site \#3 was significantly different from site \#5, \#11, \#12, and \#15. Maximum Tw at site \#4 was significantly different from site \#5, \#11, \#12, and \#15. Maximum Tw at site \#5 was significantly different from site \#17. Maximum Tw at site \#17 was significantly different from site \#5, \#6, \#11, \#12, \#14, \#15, \#18, \#19, \#21 (Table 4). 
Table 3. Descriptive statistics of stream water temperature $\left({ }^{\circ} \mathrm{C}\right)$, annually by site, quarterly (all sites), and monthly (all sites) collected in West Run Watershed, West Virginia, USA, over the 2018 annual year.

\begin{tabular}{ccccc}
\hline Sites/Season/Month & Mean & Standard Deviation & Minimum Maximum \\
\hline Site \#1 & 10.9 & 4.4 & -0.3 & 22.2 \\
Site \#2 & 10.5 & 5.4 & -0.2 & 20.6 \\
Site \#3 & 10.8 & 6.1 & -0.5 & 21.6 \\
Site \#4 & 10.5 & 6.7 & -1.9 & 22.8 \\
Site \#5 & 11.9 & 7.6 & -0.6 & 27.4 \\
Site \#6 & 11.7 & 7.1 & -0.7 & 25.9 \\
Site \#7 & 11.2 & 6.7 & -2.9 & 23.2 \\
Site \#8 & 11.5 & 6.0 & -1.1 & 22.3 \\
Site \#9 & 11.8 & 6.5 & -1.2 & 23.4 \\
Site \#10 & 11.3 & 7.1 & -1.1 & 23.5 \\
Site \#11 & 12.5 & 6.2 & 1.2 & 27.3 \\
Site \#12 & 11.3 & 7.9 & -1.3 & 27.4 \\
Site \#13 & 11.4 & 7.0 & -1.3 & 23.7 \\
Site \#14 & 11.4 & 7.5 & -1.1 & 26.4 \\
Site \#15 & 12.4 & 6.7 & -1.1 & 24.1 \\
Site \#16 & 10.8 & 7.0 & -3.0 & 26.7 \\
Site \#17 & 10.1 & 6.9 & -1.4 & 21.5 \\
Site \#18 & 12.0 & 7.3 & -0.9 & 24.8 \\
Site \#19 & 11.8 & 7.6 & -1.1 & 26.3 \\
Site \#20 & 11.3 & 7.6 & -2.0 & 23.8 \\
Site \#21 & 11.7 & 7.7 & -1.2 & 25.8 \\
Site \#22 & 11.7 & 7.2 & -0.6 & 22.3 \\
\hline Quarter 1 & 3.2 & 3.8 & -3.0 & 14.0 \\
Quarter 2 & 14.0 & 4.7 & 1.6 & 27.3 \\
Quarter 3 & 19.2 & 2.2 & 12.5 & 27.4 \\
Quarter 4 & 8.5 & 4.6 & -1.4 & 22.8 \\
\hline January & 1.4 & 2.3 & -3.0 & 8.3 \\
February & 5.5 & 3.2 & -2.6 & 14.0 \\
March & 4.6 & 2.5 & -1.4 & 13.2 \\
April & 4.6 & 2.5 & -1.4 & 13.2 \\
May & 9.2 & 3.3 & 1.6 & 23.4 \\
June & 16.1 & 2.3 & 9.0 & 24.2 \\
July & 18.0 & 2.5 & 10.3 & 27.4 \\
August & 19.3 & 2.1 & 12.8 & 26.3 \\
September & 19.6 & 1.9 & 13.2 & 27.1 \\
October & 18.2 & 2.1 & 12.5 & 26.7 \\
November & 7.4 & 2.7 & 1.0 & 15.3 \\
December & 4.9 & 2.2 & -1.4 & 11.4 \\
\hline Annual & 11.4 & 6.9 & -3.0 & 27.4 \\
& & & & \\
& & & \\
\hline
\end{tabular}


Table 4. Site by site comparison of annual maximum (parenthetic) daily Tw, along with dominant LULC, at twenty-one sites at West Run Watershed, West Virginia, USA, over the 2018 annual year. Mix Dev represents the LULC type mixed development. Only significant $(\alpha=0.05)$ results from the analysis are shown.

\begin{tabular}{|c|c|c|c|}
\hline \multicolumn{3}{|c|}{ Site Comparison } & \multirow{2}{*}{$\begin{array}{c}p \text {-Value } \\
(\alpha=0.05) \\
0.02\end{array}$} \\
\hline WRW $1\left(22.2^{\circ} \mathrm{C}\right) \operatorname{Mix} \operatorname{Dev}(53.2 \%)$ & vs. & WRW $5\left(27.4^{\circ} \mathrm{C}\right)$ Forested $(51.1 \%)$ & \\
\hline \multirow{13}{*}{ WRW $2\left(20.6^{\circ} \mathrm{C}\right)$ Forested $(74.2 \%)$} & vs. & WRW $11\left(27.3^{\circ} \mathrm{C}\right)$ Agriculture (41.9\%) & 0 \\
\hline & vs. & WRW 12 (27.4) Forested (34.5\%) & 0 \\
\hline & vs. & WRW $15\left(24.1^{\circ} \mathrm{C}\right) \mathrm{Mix} \operatorname{Dev}(70.3 \%)$ & 0.01 \\
\hline & vs. & WRW $5\left(27.4^{\circ} \mathrm{C}\right)$ Forested $(51.1 \%)$ & 0 \\
\hline & vs. & WRW $6\left(25.9^{\circ} \mathrm{C}\right)$ Forested $(58.7 \%)$ & 0 \\
\hline & vs. & WRW $9\left(23.4^{\circ} \mathrm{C}\right)$ Forested $(52.8 \%)$ & 0.01 \\
\hline & vs. & WRW $11\left(27.3^{\circ} \mathrm{C}\right)$ Agriculture (41.9\%) & 0 \\
\hline & vs. & WRW $12\left(27.4^{\circ} \mathrm{C}\right)$ Forested $(34.5 \%)$ & 0 \\
\hline & vs. & WRW $14\left(26.4^{\circ} \mathrm{C}\right)$ Forested (56.9\%) & 0 \\
\hline & vs. & WRW $15\left(24.1^{\circ} \mathrm{C}\right) \operatorname{Mix} \operatorname{Dev}(70.3 \%)$ & 0 \\
\hline & vs. & WRW $18\left(24.8^{\circ} \mathrm{C}\right)$ Forested $(48.9 \%)$ & 0 \\
\hline & vs. & WRW $19\left(26.3^{\circ} \mathrm{C}\right)$ Forested $(47.9 \%)$ & 0 \\
\hline & vs. & WRW $21\left(25.8^{\circ} \mathrm{C}\right)$ Forested $(42.2 \%)$ & 0.01 \\
\hline \multirow[t]{4}{*}{ WRW $3\left(21.6^{\circ} \mathrm{C}\right)$ Forested (61.3\%) } & vs. & WRW $5\left(27.4^{\circ} \mathrm{C}\right)$ Forested $(51.1 \%)$ & 0.01 \\
\hline & vs. & WRW $11\left(27.3^{\circ} \mathrm{C}\right)$ Agriculture (41.9\%) & 0 \\
\hline & vs. & WRW $12\left(27.4^{\circ} \mathrm{C}\right)$ Forested $(34.5 \%)$ & 0 \\
\hline & vs. & WRW $15\left(24.1^{\circ} \mathrm{C}\right) \operatorname{Mix} \operatorname{Dev}(70.3 \%)$ & 0.01 \\
\hline \multirow[t]{4}{*}{ WRW $4\left(22.8^{\circ} \mathrm{C}\right)$ Forested $(59 \%)$} & vs. & WRW $5\left(27.4^{\circ} \mathrm{C}\right)$ Forested $(51.1 \%)$ & 0.01 \\
\hline & vs. & WRW $11\left(27.3^{\circ} \mathrm{C}\right)$ Agriculture (41.9\%) & 0 \\
\hline & vs. & WRW $12\left(27.4^{\circ} \mathrm{C}\right)$ Forested (34.5\%) & 0 \\
\hline & vs. & WRW $15\left(24.1^{\circ} \mathrm{C}\right)$ Forested $(85.8 \%)$ & 0.01 \\
\hline WRW $5\left(27.4^{\circ} \mathrm{C}\right)$ Forested (51.1\%) & vs. & WRW $17\left(21.5^{\circ} \mathrm{C}\right)$ Forested $(85.8 \%)$ & 0 \\
\hline WRW $6\left(25.9^{\circ} \mathrm{C}\right)$ Forested (58.7\%) & vs. & WRW $17\left(21.5^{\circ} \mathrm{C}\right)$ Forested $(85.8 \%)$ & 0.01 \\
\hline WRW $11\left(27.3^{\circ} \mathrm{C}\right)$ Agriculture (41.9\%) & vs. & WRW $17\left(21.5^{\circ} \mathrm{C}\right)$ Forested (85.8\%) & 0 \\
\hline WRW $12\left(27.4^{\circ} \mathrm{C}\right)$ Forested $(34.5 \%)$ & vs. & WRW $17\left(21.5^{\circ} \mathrm{C}\right)$ Forested $(85.8 \%)$ & 0 \\
\hline WRW $14\left(26.4^{\circ} \mathrm{C}\right)$ Forested $(56.9 \%)$ & vs. & WRW $17\left(21.5^{\circ} \mathrm{C}\right)$ Forested $(85.8 \%)$ & 0.01 \\
\hline WRW $15\left(24.1^{\circ} \mathrm{C}\right) \operatorname{Mix} \operatorname{Dev}(70.3 \%)$ & vs. & WRW $17\left(21.5^{\circ} \mathrm{C}\right)$ Forested $(85.8 \%)$ & 0 \\
\hline \multirow[t]{3}{*}{ WRW $17\left(21.5^{\circ} \mathrm{C}\right)$ Forested $(85.8 \%)$} & vs. & WRW $18\left(24.8^{\circ} \mathrm{C}\right)$ Forested $(48.9 \%)$ & 0.01 \\
\hline & vs. & WRW $19\left(26.9^{\circ} \mathrm{C}\right)$ Forested $(47.9 \%)$ & 0.02 \\
\hline & vs. & WRW $21\left(25.8^{\circ} \mathrm{C}\right)$ Forested (42.2\%) & 0.02 \\
\hline
\end{tabular}




\section{Quarterly Stream Water Temperature}

Seasonal Tw regimes showed that quarter $1\left(\right.$ January $1^{\text {st }}-$ March $31^{\text {st }}$ ) had the lowest minimum $\mathrm{Tw}\left(-3.0^{\circ} \mathrm{C}\right.$, Site \#16), a mean of $3.2^{\circ} \mathrm{C}$, a maximum $\mathrm{Tw}$ of $14.0^{\circ} \mathrm{C}$ (Site \#12), and a standard deviation of $3.8{ }^{\circ} \mathrm{C}$ (Table 3, Figure 4). Quarter 2 (April $1^{\text {st }}-$ June $30^{\text {th }}$ ) included the highest standard deviation $\left(4.7^{\circ} \mathrm{C}\right.$ ), and a mean $\mathrm{Tw} 14.6{ }^{\circ} \mathrm{C}$, a maximum Tw of $27.3{ }^{\circ} \mathrm{C}$ (Site \#11), and a minimum Tw of $1.6^{\circ} \mathrm{C}$ (Site \#17). Quarter 3 (July $1^{\text {st }}-$ September $30^{\text {th }}$ ) had the highest mean Tw $\left(19.2{ }^{\circ} \mathrm{C}\right)$ and maximum $\mathrm{Tw}\left(27.4{ }^{\circ} \mathrm{C}\right)\left(\right.$ Site \#12) with a standard deviation of $2.2{ }^{\circ} \mathrm{C}$, and a minimum of $12.5^{\circ} \mathrm{C}$ (Site \#4).

Significant Spearman's correlation coefficients $(\alpha=0.05)$ between LULC types and maximum Tw occurred during quarter 1, 3, and 4 (Table 5). Agriculture LULC showed a significant positive correlation to maximum Tw during quarter $1(\mathrm{p}=0.03)$ and quarter $3(\mathrm{p}=0.01)$. Forested LULC showed a significant negative correlation to maximum Tw during quarter $3(\mathrm{p}=0.04)$.

The Kruskal Wallis ANOVA showed maximum daily Tw across all 21 sites differed significantly $(\mathrm{p}=0.00)$. The post hoc analysis showed that the most significant differences were observed in quarter 3 with 130 significant differences between sites (all, $\mathrm{p} \leq 0.04$ ). Quarter 4 had three significant differences, the lowest number of returned significant differences of all four quarters. All three of the returned significant differences involved site \#1 (vs. site \#4 ( $\mathrm{p}=0.01$ ), vs. $\# 5(\mathrm{p}=0.05)$, vs. \#17 ( $\mathrm{p}=0.00))$. 

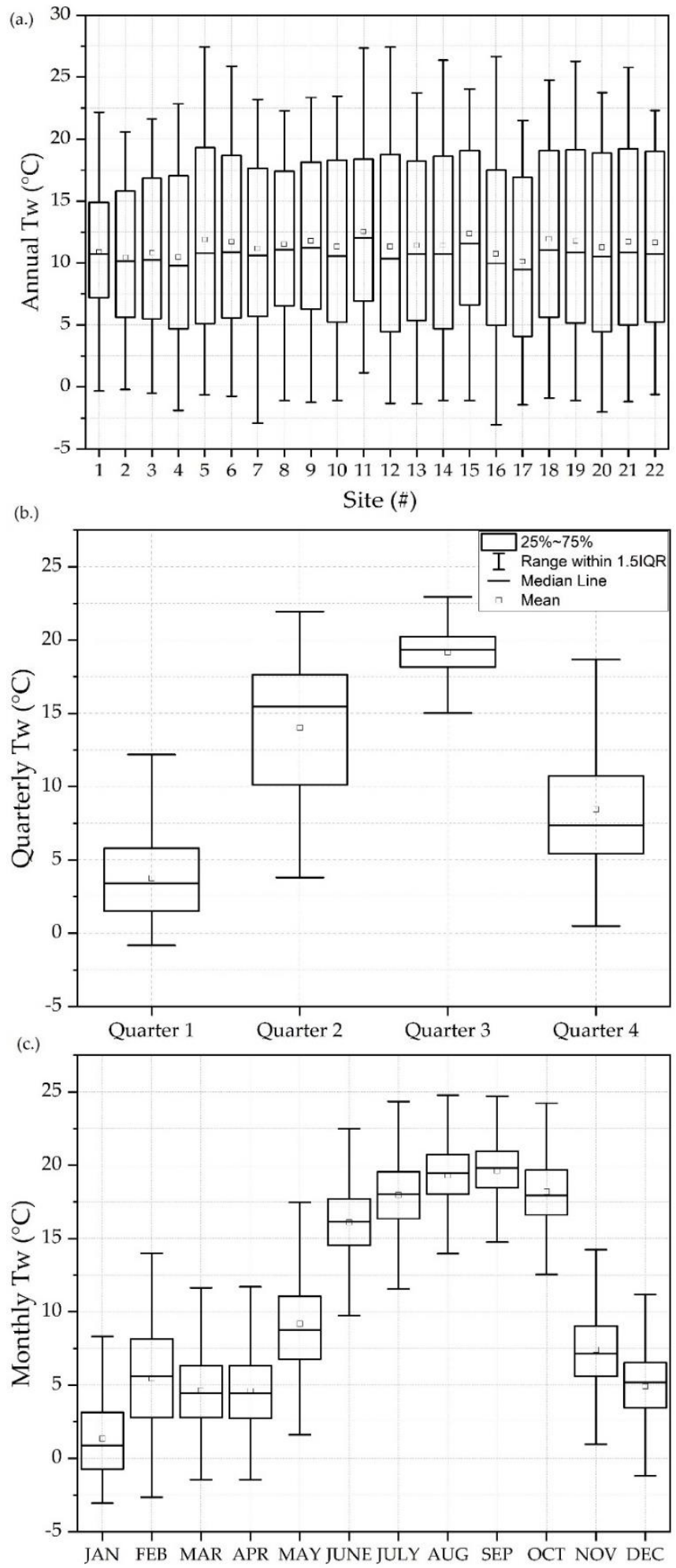

Figure 4. Five-minute water temperature data shown on a (a) site by site, (b) quarterly, and (c) monthly basis collected from 22 gauging sites in West Run Watershed, West Virginia, USA; during the 2018 annual year. 


\section{Monthly Stream Water Temperature}

January included the lowest minimum $\mathrm{Tw}\left(-3.0^{\circ} \mathrm{C}\right)($ Site \#16), with a standard deviation of $2.4{ }^{\circ} \mathrm{C}$ (all 21 sites), and a mean Tw of $1.4{ }^{\circ} \mathrm{C}$ (all 21 sites), and a maximum of $8.3{ }^{\circ} \mathrm{C}$ (Site \#8) (Table 3). May included the highest standard deviation $\left(3.3{ }^{\circ} \mathrm{C}\right)$, with a mean $\mathrm{Tw}$ of $21.8^{\circ} \mathrm{C}$, a maximum of $23.4{ }^{\circ} \mathrm{C}$ (Site \#12), and a minimum of $1.6^{\circ} \mathrm{C}$ (Site \#17). July included the highest maximum $\mathrm{Tw}\left(27.4^{\circ} \mathrm{C}\right.$ ) (Site \#12), with a mean Tw of $16.1^{\circ} \mathrm{C}$, a minimum Tw of $10.3{ }^{\circ} \mathrm{C}$ (Site \#17), and a standard deviation of $2.3{ }^{\circ} \mathrm{C}$. September included the highest mean $\mathrm{Tw}\left(19.6{ }^{\circ} \mathrm{C}\right)$, a minimum Tw of $13.2{ }^{\circ} \mathrm{C}$ (Site \#17), a maximum Tw of $27.1{ }^{\circ} \mathrm{C}$ (Site \#16), and a standard deviation of $1.9^{\circ} \mathrm{C}$ (Table 3$)$.

Significant spearman's correlation coefficients $(\alpha=0.05)$ between LULC types and maximum Tw occurred during February, April, June, July, August, September, October, November, and December. Agriculture LULCs showed a significance positive correlation to maximum Tw during February ( $\mathrm{p}=0.03$ ), June ( $\mathrm{p}=0.03$ ), August, $(\mathrm{p}=0.04)$, September $(\mathrm{p}=0.05)$, November $(\mathrm{p}=0.03)$, and December $(\mathrm{p}=0.05)$. Forested LULCs showed a significance negative correlation to maximum Tw during August ( $\mathrm{p}=0.01$ ), September $(\mathrm{p}=0.00)$, and December $(\mathrm{p}=0.02)$. Mixed development LULCs showed a significant positive correlation to maximum $\mathrm{Tw}$ during August, $(\mathrm{p}=0.01)$, September $(\mathrm{p}=0.00)$, and November $(\mathrm{p}=0.04)$ (Table 5).

LULC and Tw analysis using all sites showed 12 significant $(\alpha=0.05)$ correlations to Tw variables. The Kruskal Wallis ANOVA showed maximum daily Tw across all 21 sites differed significantly during all twelve months $(\mathrm{p}=0.00)$. The post hoc analysis showed the most significant differences occurred in July, with 108 significant differences $(\mathrm{p} \leq 0.04)$. Both February and October had no significant differences between sites (Appendix C). 
Table 5. Spearman correlations between LULC types and maximum Tw of all 21 sites, mainstem, and tributaries of West Run Watershed, West Virginia, USA, during the 2018 annual year. Positive correlations indicate an increase in Tw with increasing LULC and negative correlations indicate a decrease in Tw with increasing LULC. Bold values are significant correlations at $\alpha=0.05$. $\mathrm{Q}-$ quarter.

\begin{tabular}{|c|c|c|c|c|c|c|c|c|c|c|c|c|c|c|c|c|}
\hline & & & & & & & All S & & & & & & & & & \\
\hline & $\mathrm{Q} 1$ & Q 2 & Q3 & Q 4 & Jan & Feb & Mar & Apr & May & Jun & Jul & Aug & Sep & Oct & Nov & Dec \\
\hline $\begin{array}{c}\text { Mixed } \\
\text { Development }\end{array}$ & 0.0 & 0.1 & 0.0 & -0.1 & 0.2 & 0.1 & 0.0 & 0.0 & 0.2 & 0.1 & 0.1 & 0.2 & 0.2 & -0.1 & 0.3 & 0.3 \\
\hline Agriculture & 0.5 & 0.4 & 0.5 & 0.4 & 0.3 & 0.5 & 0.3 & 0.3 & 0.3 & 0.4 & 0.5 & 0.5 & 0.4 & 0.4 & 0.3 & 0.5 \\
\hline Forested & -0.1 & -0.2 & -0.5 & -0.3 & -0.3 & -0.2 & -0.3 & -0.1 & -0.3 & -0.2 & -0.3 & -0.4 & -0.5 & -0.3 & -0.4 & -0.5 \\
\hline
\end{tabular}

Mainstem

\begin{tabular}{|c|c|c|c|c|c|c|c|c|c|c|c|c|c|c|c|c|}
\hline & Q 1 & Q 2 & Q3 & Q 4 & Jan & Feb & Mar & Apri & May & Jun & July & Aug & Sep & Oct & Nov & Dec \\
\hline $\begin{array}{c}\text { Mixed } \\
\text { Development }\end{array}$ & 0.0 & 0.4 & 0.5 & 0.4 & -0.6 & 0.0 & 0.2 & 0.6 & 0.6 & 0.4 & 0.5 & 0.9 & 0.9 & 0.4 & 0.6 & -0.1 \\
\hline Agriculture & 0.5 & 0.3 & 0.5 & 0.8 & -0.2 & 0.5 & 0.4 & 0.5 & 0.4 & 0.3 & 0.5 & 0.7 & 0.7 & 0.8 & 0.8 & 0.7 \\
\hline Forested & -0.3 & -0.5 & -0.6 & -0.6 & 0.5 & -0.3 & -0.4 & -0.7 & -0.7 & -0.5 & -0.6 & -0.9 & -0.9 & -0.6 & -0.7 & -0.2 \\
\hline
\end{tabular}

Tributaries

\begin{tabular}{|c|c|c|c|c|c|c|c|c|c|c|c|c|c|c|c|c|}
\hline & $\mathrm{Q} 1$ & Q 2 & Q3 & Q 4 & Jan & Feb & Mar & Apr & May & Jun & Jul & Aug & Sep & Oct & Nov & Dec \\
\hline $\begin{array}{c}\text { Mixed } \\
\text { Development }\end{array}$ & 0.0 & 0.1 & 0.1 & -0.2 & 0.2 & 0.1 & -0.1 & -0.3 & 0.3 & 0.3 & 0.4 & 0.3 & 0.3 & 0.1 & 0.6 & 0.4 \\
\hline Agriculture & 0.5 & 0.3 & 0.4 & 0.3 & -0.1 & 0.4 & 0.2 & 0.2 & 0.5 & 0.6 & 0.6 & 0.6 & 0.6 & 0.5 & 0.6 & 0.4 \\
\hline Forested & 0.0 & 0.0 & -0.4 & -0.1 & -0.4 & 0.4 & 0.2 & 0.4 & 0.3 & 0.5 & 0.3 & 0.2 & 0.1 & 0.3 & 0.4 & 0.2 \\
\hline
\end{tabular}

PCA

As stated previously, PCAs included in this work are for illustrative purposes, strictly. Given the nature of data used in this analysis, further elaboration of each Principle component's composition will not be included. Annual time-series comparisons between LULC areas and Tw variables all showed two Eigenvalues above the threshold of importance. If all variables $(n=4)$ exerted equal influence for the annual PCA the loading values would be 0.5 (i.e., $\sqrt{ }(1 / \mathrm{n})$ ) (Bro and Smilde, 2014; Kellner et al., 2018). For mean Tw annual PCA, PC1 had an Eigenvalue of 2.0 and explained 50.4\% of the variance and PC2 had an Eigenvalue of 1.2 and explained 31.0\% of the variance (Figure 7). For the minimum Tw annual PCA, PC1 had an Eigenvalue of 1.8 and explained $45.1 \%$ of the variance and PC2 had an Eigenvalue of 1.2 and explained $30.5 \%$ of the 
variance. For the maximum Tw annual PCA, PC1 had an Eigenvalue of 1.8 and explained 45.8\% of the variance and PC2 had an Eigenvalue of 1.7 and explained $41.8 \%$ of the variance.

Mean and maximum Quarterly time-series comparisons between LULC areas showed three Eigenvalues above the threshold of importance. The minimum Tw Quarterly time-series comparisons between LULC areas showed two Eigenvalues above the threshold of importance. If all variables $(n=7)$ exerted equal influence the loading values would equal 0.38 . For the mean Tw quarterly PCA, PC1 had an Eigenvalue of 2.9 and explained 40.9\% of the variance and PC2 had an Eigenvalue of 2.0 and explained $28.4 \%$ of the variance. For the minimum Tw quarterly PCA, PC1 had an Eigenvalue of 2.5 and explained $35.3 \%$ of the variance and PC2 had an Eigenvalue of 1.8 and explained $25.6 \%$ of the variance. For the maximum Tw quarterly PCA, PC1 had an Eigenvalue of 3.4 and explained $47.9 \%$ of the variance and PC2 had an Eigenvalue of 1.8 and explained $26.3 \%$ of the variance.

Monthly time-series comparison between LULC areas and Tw variables all showed four Eigenvalues above the threshold of importance (Appendix D). If all variables $(n=15)$ exerted equal influence the loading values would equal 0.26. For the mean Tw monthly PCA, PC1 had an Eigenvalue of 6.2 and explained $41.3 \%$ of the variance and PC2 had an Eigenvalue of 5.5 and explained $36.8 \%$ of the variance. For the minimum Tw monthly PCA, PC1 had an Eigenvalue of 6.4 and explained $42.9 \%$ of the variance and PC2 had an Eigenvalue of 3.4 and explained 22.6\% of the variance. For the maximum Tw monthly PCA, PC1 had an Eigenvalue of 7.5 and explained $49.7 \%$ of the variance and PC2 had an Eigenvalue of 2.7 and explained $17.8 \%$ of the variance. 


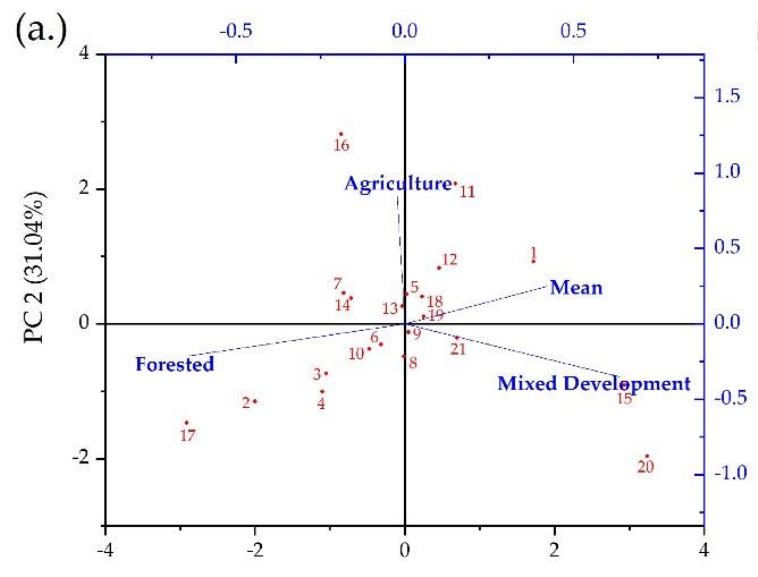

PC $1(50.41 \%)$

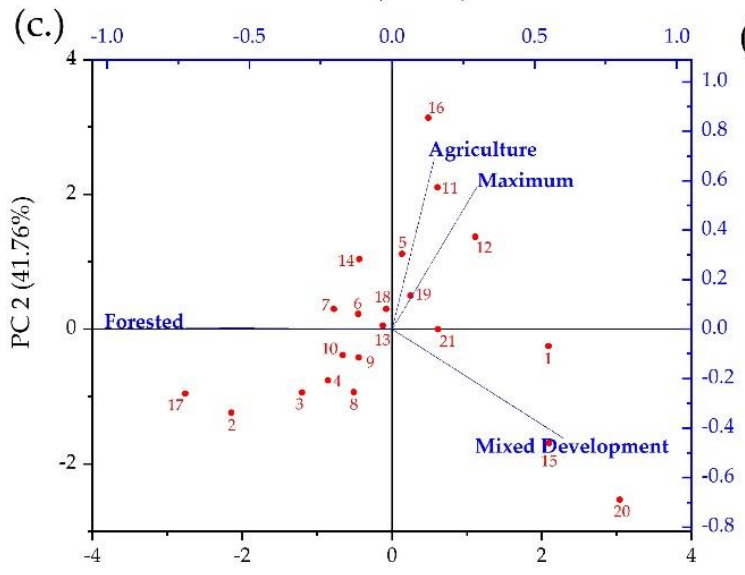

PC $1(45.79 \%)$

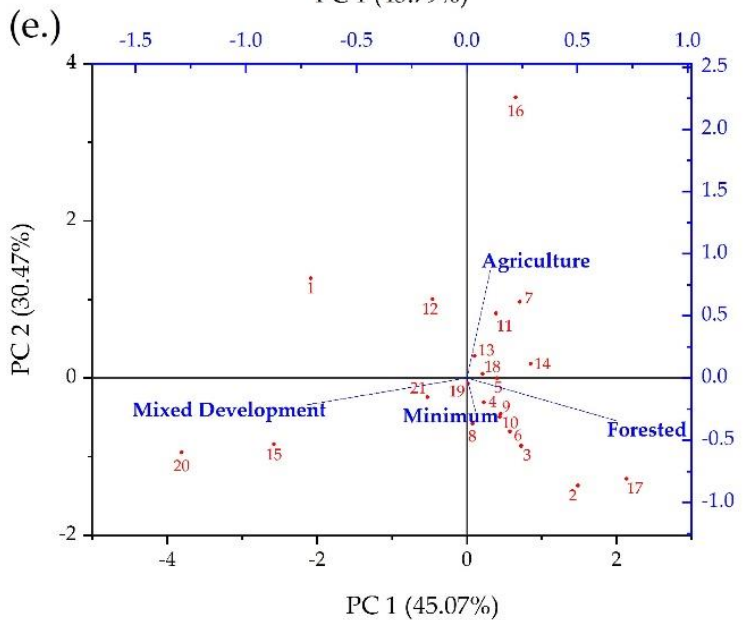

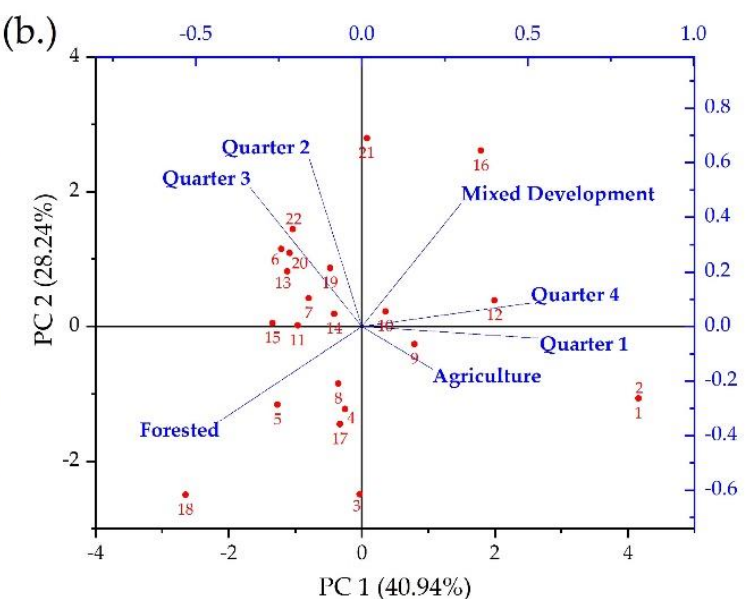

(d.)

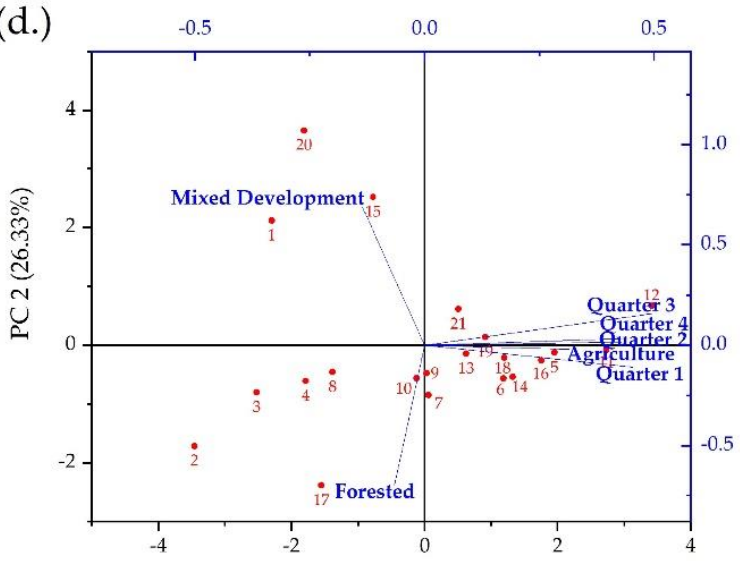

PC $1(47.88 \%)$

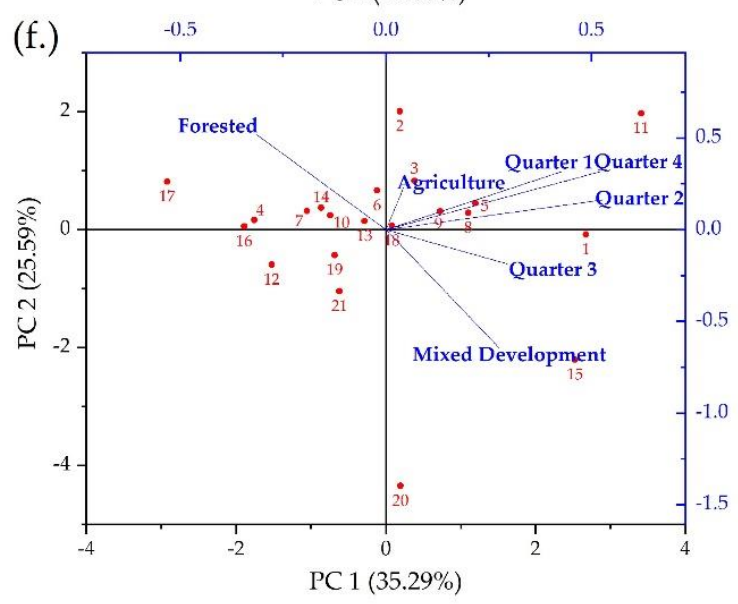

Figure 5. Results of principle component analysis, showing biplots of extracted principle components of annual water temperature data (mean (a), maximum (c), and minimum (e)), and biplots with extracted principle components of quarterly water temperature data (mean (b), maximum (d), and minimum (f)) collected during the 2018 annual year at the 21 monitoring sites of West Run Watershed, WV, USA; and their corresponding LULC area $\left(\mathrm{km}^{2}\right)$. 


\section{Discussion}

\section{Climate during Study}

Climate variables (e.g., Ta, precipitation, net radiation) recorded in West Run Watershed over the 2018 annual year were within the range of historic climate of West Virginia (i.e., 19002016) (Kutta and Hubbart, 2019a). Average mean temperature (11.6 $\left.{ }^{\circ} \mathrm{C}\right)$ differed only slightly (1.7\%) from historic (1900-2016) averages observed in West Virginia from 1900-2016 (11.4 $\left.{ }^{\circ} \mathrm{C}\right)$. Typical of the climate in West Run Watershed, no dry season was observed during the 2018 annual year, but somewhat more of the total precipitation fell during quarters two and three that in one and four (Petersen and Hubbart, 2020). However, during the study period there was above average (20.5\% higher) total precipitation relative to the historic (1900-2016) average (1096 mm) ("Fourth National Climate Assessment," n.d.). Thus, the 2018 findings may not be representative of average precipitation years.

\section{Stream Water Temperature LULC Relations}

The null hypotheses of hypothesis 1,2 , and 3 were rejected given results from the TwLULC analyses and the site-by-site analysis. In general, results from the Spearman's analysis (Table 5, Appendix E) showed that an increase in the proportion of forested LULC types is negatively correlated to mean and maximum $\mathrm{Tw}$ at all three temporal scales (i.e., annual, quarterly, monthly) as confirmed in previous studies (Beschta and Taylor, 1988; Johnson and Jones, 2011; Moore et al., 2006). These results follow the same conclusions made by previous researchers that that showed that forest harvest (e.g., clear-cuts / canopy removal) increase Tw (Gravelle and Link, 2007; Johnson and Jones, 2011; Moore et al., 2006; Pollock et al., 2009). Additionally, although not surprising, during the winter season, a positive correlation was observed between the proportion of forest LULC and minimum Tw. This correlation was likely related to canopy insulation during winter months (Moore et al, 2006; Oke, 1987). Moore et al. ( 2005) suggested that riparian vegetation insulates Tw by lowering convective heat loss to the above atmosphere, and thus warmer Tw. In the current work, positive correlations between maximum Tw and percent forest LULC were observed in specific tributaries (Table 5). These results contradict findings of previous research (Beschta and Taylor, 1988; Moore et al., 2005; 
Pollock et al., 2009) which found as forested LULC proportions increase Tw decreases. However, when sites 8 and 9 were removed from the data pool, correlations trends reverted to the expected negative correlation (Beschta and Taylor, 1988; Moore et al., 2005; Pollock et al., 2009). For sites 8 and 9 of the current work, the positive correlation may be attributed to the high proportion forest in the subcatchment but mixed development LULC types at both sites 8 and 9 directly adjacent to the stream.

Results from the Spearman's analysis (Table 5, Appendix E) showed mixed development LULC types were significantly correlated to mean, minimum, and maximum Tw with variable effects throughout the year. While results of the mixed development-Tw analysis were similar to results of past research, the high number of both sampling sites and sampling frequency of this study provide needed validation for results of previous research (Rice et al., 2011; Zeiger et al., 2016; Zeiger and Hubbart, 2015). Interestingly, Spearman's results (Table 5, Appendix E) showed a negative correlation between mixed development LULC types and minimum, maximum, and mean Tw during the winter and early spring months (e.g., January, February, March, April, November, December), indicating overall lower Tw. These findings contradict results of other research analyzing the relationship between mixed development LULC and Tw. Rice et al. (2011) showed that mean Tw increased during the winter season in heavily urbanized catchments of Boone, North Carolina. Alternatively to Rice et al. (2011), lower Tw correlated to mixed development during cooler months (e.g., January, February, March, April, November, December) could be explained by runoff from impervious surfaces, which during these months is often lower in temperature than Tw of surrounding streams. Qun et al. (2016) showed a negative correlation between urban impervious surface and land surface temperatures (i.e., lower surface temperatures) during winter daytimes. Conflicting results might be further explained by the complex physiographic mosaic of the study watershed in which mixed development LULC types are broken up by forested and agricultural LULC types at varying relative positions on the landscape, thus leaving room for future investigations.

Although not always significant, agricultural LULC types had a positive Spearman's correlation, with maximum Tw in every quarter and month, except January (Table 5). These findings may be, at least in part, due to increased soil shortwave radiation exposure during periods when fields are bare, increased subsurface lateral flow rates, and/or the removal of riparian vegetation as per findings of previous literature (Macedo et al., 2013; Story et al., 2003; Younus 
et al., 2000). Interestingly, a potential relationship appeared between maximum Tw and crop absence. Typically, in the study watershed, certain crops (e.g., triticale, rye, and hay) are planted in spring and harvested between May and early July (U.S. Department of Agriculture, 1997), exposing fields and potentially explaining the observed trend in Table 5. Therefore, the potential relationship between crop absence and maximum Tw could be explained, at least in part, by increased shortwave interception and reduced runoff volumes through evapotranspiration and interception of precipitation by crops (Oke, 1987; Xuhui, 2018). In addition, after harvest the relationship becomes significant because runoff volumes increase and the soil is exposed to greater amounts of shortwave radiation thereby reaching higher temperatures (Hatfield et al., 2001; Oke, 1987; Younus et al., 2000). Furthermore, during these periods water contacting or infiltrating into the soil is heated and transported to surrounding streams through subsurface lateral flows (Younus et al., 2000).

\section{LULC Tw Tipping Points}

Decreasing forested LULC proportion influenced Tw variables in the current investigation. As the percent of forested LULC types decreased below 74.2\%, associated maximum Tw began to increase (thus a potential tipping point). Below 61.1\%, Forested LULC mean Tw began to either increase or decrease depending on the time of year (Table 5., Appendix E), and below 52.2\% Forested LULC the minimum Tw of associated streams began to increase or decrease again depending on the time of year. The increase or decrease is based on the significant Spearman's correlations which vary quarterly and monthly (Table 5., Appendix E). Mixed development LULC types were the second LULC to influence Tw. 


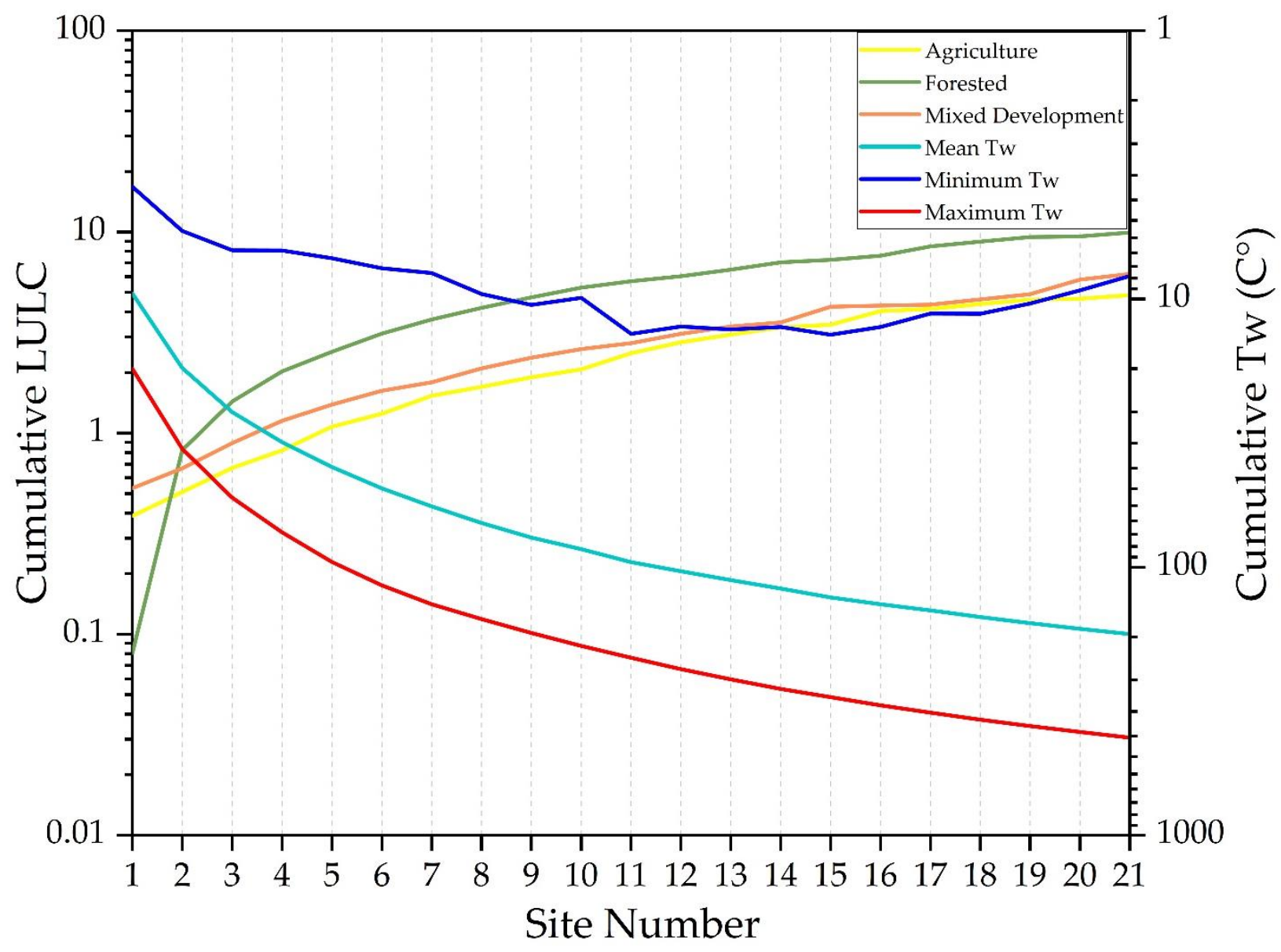

Figure 6. Average annual $\mathrm{Tw}$ variables (mean $\mathrm{Tw}$, minimum $\mathrm{Tw}$, and maximum $\mathrm{Tw}$ ) vs.

Cumulative LULC types (forested, agriculture, and mixed development) ( $1=100 \%)$ moving from the headwaters to the terminus in West Run Watershed, West Virginia, USA, during the 2018 annual year.

As the percent of mixed development LULC types increased above $14 \%$, associated mean Tw increased (thus a potential mixed development tipping point). Above $24.1 \%$ mixed development maximum Tw began to either increase or decrease depending on the time of year, and above $26.8 \%$ mixed development the minimum $\mathrm{Tw}$ of streams began to increase or decrease (depending on the time of year). Agricultural LULC types also influenced Tw significantly. As the percent of agriculture LULC types increased above $14.9 \%$ associated maximum Tw began to increase. Above $16.0 \%$ agriculture LULC mean Tw begins increase and above $26.4 \%$ agriculture LULC the minimum stream Tw began to increase or decrease (time of year dependent). 


\section{Summary and Conclusion}

Influencing both abiotic and biotic processes, $\mathrm{Tw}$ is a water quality parameter of interest to land use managers, policy makers, and water quality experts. Tw influences abiotic processes such as the settling rates of suspended partials and biotic processes such as gas exchange rates in aquatic organisms (Boyde et al., 2016). Although the subject of Tw is a frequent topic of past literature the relation to land-use in a contemporary mixed land-use watershed is largely unknown. Research over Tw relations to surrounding land use is often limited to proximal areas (i.e., riparian buffer) to the waterbody being researched or conducted in watersheds consisting of a homogenous LULC type. The homogenous LULC assumption is often not viable due to the contemporary nature of anthropogenic development and expansion in many watersheds. Instead most watersheds are closer to the complex mosaic of LULC types in a contemporary mixed landuse watershed like West Run Watershed. Therefore, research in watersheds like West Run can provide insight into LULC change impacts on water quality parameters such as Tw. No previous research investigating stream water temperature $(\mathrm{Tw})$ in contemporary watersheds has used such a high temporal and spatial sampling regime as that included in the current investigation. In addition, using a paired and nested-scale experimental watershed study design allowed for separation of the watershed into subsections designated with associated land-use practices. Implementation of the experimental design used in the current research is necessary to provide both validation for previous results and the discovery of temporal variation in LULC-Tw integrated processes. In the current work, the relationship between LULC types and Tw was investigated in the Appalachian region of the eastern United States. The analysis used fiveminute Tw time-series data collected at 21 nested sites using an experimental watershed study design. Results indicated that LULC has varying effects on Tw both spatially and temporally. In this research, the forested LULC type displayed a negative correlation to mean and maximum Tw in the spring and summer months. Throughout the year forested LULC type displayed an overall lowered $\mathrm{Tw}$ variability. A significant $(\mathrm{p}=0.01$ ) positive relation between agricultural LULCs and maximum water temperature was observed during this research. LULC types associated with the agricultural classification are often associated with the removal of riparian buffer strips and the exposure of soil to shortwave radiation for some period when no crops are present during the annual year (Borman and Larson, 2003b; Wiebe and Gollehon, 2007; Younus et al., 2000). Both factors shown in previous research to increase Tw through increased 
shortwave radiation exposure and increased temperature of subsurface lateral flows entering neighboring streams (Younus et al., 2000). Mixed-development LULC types, associated with impervious surfaces, are frequently observed to have a positive relation with mean, maximum, and Tw standard deviation in the literature (Kinouchi et al., 2007; Palmer and Nelson, 2007; Rice et al., 2011; Zeiger and Hubbart, 2015). The relationship between Mixed-development and Tw variables analyzed in this thesis varied throughout the year depending on the season and month, similar to findings of primary literature (Palmer and Nelson, 2007; Rice et al., 2011; Zeiger and Hubbart, 2015). During the winter months a negative correlation with minimum Tw was observed. This negative relationship correlates to an overall lower minimum Tw. This relationship is likely attributed to surface runoff being cooled when contacting impervious surfaces during these colder months (Qun et al. 2016). Alternatively, during the summer months, a positive relationship was shown between mixed-development LULC types and all Tw variables. These results correlated to elevated $\mathrm{Tw}$ with higher overall variability similar to those of previous work (Palmer and Nelson, 2007; Rice et al., 2011; Zeiger and Hubbart, 2015). Although results are specific to the study watershed, the finding of tipping points shows LULC thresholds that, when exceeded, may begin to impact associated Tw. These relationships likely exist in all watersheds, particularly contemporary municipal watersheds. Results of this thesis investigation highlighted the variable temporal impacts surrounding LULC types can have on Tw in a contemporary mixed land-use watershed. Many of these results were likely only elucidated due to the high temporal and spatial resolution of data collected at the twenty-one sites in West Run Watershed. Conclusions made by past researchers were both supported and expanded upon through the interpretation and analysis of these data. The alternative hypotheses of this thesis, established through results of other Tw literature, were supported. These results will advance decision making success of land managers and policymakers concerned with the health of aquatic ecosystems. In particular, the high-resolution $(n=21)$ study design presented in this work facilitates identification of upland mitigation sites and corresponding greater certainty in fiscal investment outcomes. 


\section{References}

Anderson, W.P., Anderson, J.L., Thaxton, C.S., Babyak, C.M., 2010. Changes in stream temperatures in response to restoration of groundwater discharge and solar heating in a culverted, urban stream. Journal of Hydrology 393, 309-320. https://doi.org/10.1016/j.jhydrol.2010.08.030

Arguez, A., Durre, I., Applequist, S., Vose, R.S., Squires, M.F., Yin, X., Heim, R.R., Owen, T.W., 2012. NOAA's 1981-2010 U.S. Climate Normals: An Overview. Bulletin of the American Meteorological Society 93, 1687-1697. https://doi.org/10.1175/BAMS-D-11-00197.1

Arismendi, I., Safeeq, M., Dunham, J.B., Johnson, S.L., 2014. Can air temperature be used to project influences of climate change on stream temperature? Environmental Research Letters 9. https://doi.org/10.1088/1748-9326/9/8/084015

Beitinger, T.L., Bennett, W.A., Mccauley, R.W., 2000. Temperature tolerances of North American freshwater fishes exposed to dynamic changes in temperature. Environmental Biology of Fishes 58, 237-275.

Beschta, R.L., Taylor, R.L., 1988. Stream Temperature Increases and Land Use in a Forested Oregon Watershed. JAWRA Journal of the American Water Resources Association 24, 19-25. https://doi.org/10.1111/j.1752-1688.1988.tb00875.x

Blackwelder, E., 1914. A summary of the orogenic epochs in the geological history of North America. J. Geol. XXII, 633-654.

Bogan, T., Mohseni, O., Stefan, H.G., 2003. Stream temperature-equilibrium temperature relationship. Water Resources Research 39, 1-12. https://doi.org/10.1029/2003WR002034

Booth, D.B., Roy, A.H., Smith, B., Capps, K.A., 2016. Global perspectives on the urban stream syndrome. Freshwater Science 35, 412-420. https://doi.org/10.1086/684940

Borman, M., Larson, L., 2003. A case study of river temperature response to agricultural land use and environmental thermal patterns. Journal of soil and water conservation 58, 8-12.

Boyde, C., Drinking, A., Quality, W., Main, W., Program, F., Program, C.C., Program, W.P., 2016. Water Quality An Introduction.

Bro, R., Smilde, A.K., 2014. Principal component analysis. Anal. Methods 6, 2812-2831. https://doi.org/10.1039/C3AY41907J

Brown, G.W., Krygier, J.T., 1970. Effects of Clear-Cutting on Stream Temperature. Water Resources Research 6, 1133-1139. https://doi.org/10.1029/WR006i004p01133 
Bulliner, E., Hubbart, J.A., 2013. An improved hemispherical photography model for stream surface shortwave radiation estimations in a central U.S. hardwood forest. Hydrological Processes 27, 3885-3895. https://doi.org/10.1002/hyp.9524

Caissie, D., 2006. The thermal regime of rivers: A review. Freshwater Biology 51, 1389-1406. https://doi.org/10.1111/j.1365-2427.2006.01597.x

Caldwell, P., Segura, C., Gull Laird, S., Sun, G., Mcnulty, S.G., Sandercock, M., Boggs, J., Vose, J.M., 2015. Short-term stream water temperature observations permit rapid assessment of potential climate change impacts. Hydrological Processes 29, 2196-2211. https://doi.org/10.1002/hyp.10358

Campbell, G.S., Norman, J.M., 1998. Introduction to environmental biophysics, 2nd ed. ed. Springer, New York.

Coutant, C.C., 1999. Perspectives on Temperature in the Pacific Northwest's Fresh Waters (No. ORNL/TM-1999/44, 9042). https://doi.org/10.2172/9042

Ficklin, D.L., Luo, Y., Stewart, I.T., Maurer, E.P., 2012. Development and application of a hydroclimatological stream temperature model within the Soil and Water Assessment Tool. Water Resources Research 48, 1-16. https://doi.org/10.1029/2011WR011256

Fourth National Climate Assessment, n.d. 32.

Gravelle, J.A., Link, T.E., 2007. Influence of timber harvesting on headwater peak stream temperatures in a northern Idaho watershed. (Special issue: Science and management of forest headwater streams). Forest Science 53, 189-205.

Gregory, S.V., Swanson, F.J., McKee, W.A., Cummins, K.W., 1991. An ecosystem perspective of riparian zones land and water. Bioscience 41(8), 540-551.

Grime, J.P., 1998. Benefits of plant diveristy to ecosystems: immediate, filter and founder effects. Journal of Ecology 86, 902-910.

Grime, J.P., 1973. Competitive Exclusion in Herbaceous Vegetation. Nature 242, 344-347.

Gu, R., McCutcheon, S., Chen, C.J., 1999. Development of weather-dependent flow requirements for river temperature control. Environmental Management 24, 529-540. https://doi.org/10.1007/s002679900252

Hatfield, J.L., Sauer, T.J., Prueger, J.H., 2001. Managing Soils to Achieve Greater Water Use Efficiency: A Review. AGRONOMY JOURNAL 93, 10. 
Helsel, D.R., Hirsch, R.M., 1992. Statistical methods in water resources. Statistical methods in water resources. https://doi.org/10.2307/1269385

Herb, W., Janke, B., Mohseni, O., Stefan, H., 2008. Thermal pollution of streams by runoff from paved surfaces. Hydrological Processes 22, 987-999. https://doi.org/10.1002/hyp

Herb, W.R., Stefan, H.G., 2011. Modified equilibrium temperature models for cold-water streams. Water Resources Research 47, 1-14. https://doi.org/10.1029/2010WR009586

Hewlett, J.D., Lull, H.W., Reinhart, K.G., 1969. In Defense of Experimental Watersheds. Water Resour. Res. 5, 306-316. https://doi.org/10.1029/WR005i001p00306

Houston, D., n.d. Pluvial (rain-related) flooding in urban areas: the invisible hazard.

Hubbart, J.A., Kellner, E., Zeiger, S.J., 2019. A Case-Study Application of the Experimental Watershed Study Design to Advance Adaptive Management of Contemporary Watersheds. Water 11, 2355. https://doi.org/10.3390/w11112355

Hubbart, J.A., Link, T.E., Gravelle, J.A., Elliot, W.J., 2007. Timber harvest impacts on water yield in the continental/maritime hydroclimatic region of the United States. Forest Science 53, 169-180.

Imhoff, M.L., Zhang, P., Wolfe, R.E., Bounoua, L., 2010. Remote sensing of the urban heat island effect across biomes in the continental USA. Remote Sensing of Environment 114, 504513. https://doi.org/10.1016/j.rse.2009.10.008

Johnson, M. Wilby, R., 2007. Water Resources Research. Journal of the American Water Resources Association 5, 2-2. https://doi.org/10.1111/j.1752-1688.1969.tb04897.x

Johnson, R.K., Almlöf, K., 2016. Adapting boreal streams to climate change: effects of riparian vegetation on water temperature and biological assemblages. Freshwater Science 35, 984997. https://doi.org/10.1086/687837

Johnson, S.L., Jones, J.A., 2011. Stream temperature responses to forest harvest and debris flows in western Cascades, Oregon. Canadian Journal of Fisheries and Aquatic Sciences 57, 3039. https://doi.org/10.1139/f00-109

Kellner, E., Hubbart, J., Stephan, K., Freedman, Z., Kutta, E., Kelly, C., Morrissey, E., 2018. Characterization of sub-watershed-scale stream chemistry regimes in an Appalachian mixed-land-use watershed. Environmental Monitoring and Assessment 190. https://doi.org/10.1007/s10661-018-6968-9 
Kellner, E., Hubbart, J.A., 2017a. Application of the experimental watershed approach to advance urban watershed precipitation/discharge understanding. Urban Ecosystems 20, 799-810. https://doi.org/10.1007/s11252-016-0631-4

Kellner, E., Hubbart, J.A., 2017b. Confounded by forgotten legacies: Effectively managing watersheds in the contemporary age of unknown unknowns. Hydrol. Process. 31, 28022808. https://doi.org/10.1002/hyp.11223

Kinouchi, T., Yagi, H., Miyamoto, M., 2007. Increase in stream temperature related to anthropogenic heat input from urban wastewater. Journal of Hydrology 335, 78-88. https://doi.org/10.1016/j.jhydrol.2006.11.002

Kutta, E., Hubbart, J.A., 2016. Reconsidering meteorological seasons in a changing climate. Climatic Change 137, 511-524. https://doi.org/10.1007/s10584-016-1704-3

Kutta, Hubbart, 2019a. Climatic Trends of West Virginia: A Representative Appalachian Microcosm. Water 11, 1117. https://doi.org/10.3390/w11061117

Kutta, E., Hubbart, J., 2019b. Observed Mesoscale Hydroclimate Variability of North America's Allegheny Mountains at 40.2॰ N. Climate 7, 1-24.

LeBlanc, R.T., Brown, R.D., FitzGibbon, J.E., 1997. Modeling the effects of land use change on the water temperature in unregulated urban streams. Journal of Environmental Management 49, 445-469. https://doi.org/10.1006/jema.1996.0106

Lenton, T.M., 2013. Environmental Tipping Points, Annual Review of Environment and Resources. https://doi.org/10.1146/annurev-environ-102511-084654

Liu, D., Xu, Y., Guo, S., Xiong, L., Liu, P., Zhao, Q., 2018. Stream temperature response to climate change and water diversion activities. Stochastic Environmental Research and Risk Assessment 32, 1397-1413. https://doi.org/10.1007/s00477-017-1487-8

Ma, Q., Wu, J., He, C., 2016. A hierarchical analysis of the relationship between urban impervious surfaces and land surface temperatures: spatial scale dependence, temporal variations, and bioclimatic modulation. Landscape Ecol 31, 1139-1153. https://doi.org/10.1007/s10980016-0356-Z

Macedo, M.N., Coe, M.T., DeFries, R., Uriarte, M., Brando, P.M., Neill, C., Walker, W.S., 2013. Land-use-driven stream warming in southeastern Amazonia. Philosophical Transactions of the Royal Society B: Biological Sciences 368. https://doi.org/10.1098/rstb.2012.0153 
Martin, R.W., Petty, J.T., 2009. Local stream temperature and drainage network topology interact to influence the distribution of smallmouth bass and brook trout in a Central appalachian watershed. Journal of Freshwater Ecology 24, 497-508. https://doi.org/10.1080/02705060.2009.9664323

Marzadri, A., Tonina, D., Bellin, A., 2013. Effects of stream morphodynamics on hyporheic zone thermal regime. Water Resources Research 49, 2287-2302. https://doi.org/10.1002/wrcr.20199

Menberg, K., Blum, P., Schaffitel, A., Bayer, P., 2013. Long-Term Evolution of Anthropogenic Heat Fluxes into a Subsurface Urban Heat Island. Environ. Sci. Technol. 47, 9747-9755. https://doi.org/10.1021/es401546u

Mohseni, O., Stefan, H.G., 1999. Stream temperature/air temperature relationship: A physical interpretation. Journal of Hydrology 218, 128-141. https://doi.org/10.1016/S00221694(99)00034-7

Moore, R.D., Spittlehouse, D.L., Story, A., 2005. Riparian microclimate and stream temperature response to forest harvesting: a review. Journal of the American Water Resources Association 7, 813-834.

Morgantown, West Virginia Population 2019 (Demographics, Maps, Graphs) [WWW Document], n.d.

Mustafa, M., Barnhart, B., Babbar-Sebens, M., Ficklin, D., 2018. Modeling landscape change effects on stream temperature using the Soil and Water Assessment Tool. Water (Switzerland) 10, 1-18. https://doi.org/10.3390/w10091143

Mwedzi, T., Katiyo, L., Mugabe, F.T., Bere, T., Bangira, C., Mangadze, T., Kupika, O.L., 2016. A spatial assessment of stream-flow characteristics and hydrologic alterations, post dam construction in the Manyame catchment, Zimbabwe. Water SA 42, 194-202. https://doi.org/10.4314/wsa.v42i2.03

Naiman, R., Décamps, H., 1997. The ecology of interfaces: Riparian Zones. Annual Review of Ecology, Evolution, and Systematics 28, 621-658. https://doi.org/10.1146/annurev.ecolsys.28.1.621

New, M., Lister, D., Hulme, M., Makin, I., 2002. A high-resolution data set of surface climate over global land areas. Clim. Res. 21, 1-25. https://doi.org/10.3354/cr021001 
O’Driscoll, M.A., DeWalle, D.R., 2006. Stream-air temperature relations to classify streamground water interactions in a karst setting, central Pennsylvania, USA. Journal of Hydrology 329, 140-153. https://doi.org/10.1016/j.jhydrol.2006.02.010

Oke, T.R., 1987. Boundary Layer Climates, Second. ed.

Palmer, M.A., Nelson, K.C., 2007. Stream Temperature Surges under Urbanization. Journal of the American Water Resources Association 43, 440-452. https://doi.org/10.1111/ j.17521688.2007.00034.x

Paul, M.J., Meyer, J.L., 2001. Stream In The Urban Landscape. Annual Review of Ecology and Systematics 32, 333-365. https://doi.org/10.1146/annurev.ecolsys.32.081501.114040

Petersen, F., Hubbart, J.A., 2020. Quantifying Escherichia coli and Suspended Particulate Matter Concentrations in a Mixed-Land Use Appalachian Watershed. Water 12, 532. https://doi.org/10.3390/w12020532

Petersen, F., Hubbart, J.A., Kellner, E., Kutta, E., 2018. Land-use-mediated Escherichia coli concentrations in a contemporary Appalachian watershed. Environmental Earth Sciences 77, 1-13. https://doi.org/10.1007/s12665-018-7948-7

Petty, J.T., Thorne, D., Huntsman, B.M., Mazik, P.M., 2014. The temperature-productivity squeeze: Constraints on brook trout growth along an Appalachian river continuum. Hydrobiologia 727, 151-166. https://doi.org/10.1007/s10750-013-1794-0

Pollock, M.M., Beechie, T.J., Liermann, M., E, R., 2009. Stream Temperature Relationships to Forest Harvest in the Olympic Peninsula ,. Fisheries Science 45, 1-33. https://doi.org/10.1111

Poole, G.C., Berman, C.H., 2001. An ecological perspective on in-stream temperature: Natural heat dynamics and mechanisms of human-caused thermal degradation. Environmental Management 27, 787-802. https://doi.org/10.1007/s002670010188

Raitz, K., 2019. Appalachia: A Regional Geography: Land, People, And Development. Routledge.

Ren, W., Zhong, Y., Meligrana, J., Anderson, B., Watt, W.E., Chen, J., Leung, H.L., 2003. Urbanization, land use, and water quality in Shanghai 1947-1996. Environment International 29, 649-659. https://doi.org/10.1016/S0160-4120(03)00051-5

Rentch, J.S., Adams, H.S., Anderson, J.T., Grafton, W.N., Fortney, R.H., Stephenson, S.L., 2005. Vegetation-site relationships of roadside plant communities in West Virginia, USA. 
Journal of Applied Ecology 42, 129-138. https://doi.org/10.1111/j.13652664.2004.00993.x

Rice, J.S., Anderson, Jr., W.P., Thaxton, C.S., 2011. Urbanization influences on stream temperature behavior within low-discharge headwater streams. Hydrological Research Letters 5, 27-31. https://doi.org/10.3178/hrl.5.27

Segura, C., Caldwell, P., Sun, G., Mcnulty, S., Zhang, Y., 2015. A model to predict stream water temperature across the conterminous USA. Hydrological Processes 29, 2178-2195. https://doi.org/10.1002/hyp.10357

Sinokrot, B.A., Gulliver, J.S., 2000. In-stream flow impact on river water temperatures. Journal of Hydraulic Research 38, 339-349. https://doi.org/10.1080/00221680009498315

Sinokrot, B.A., Stefan, H.G., 1993. Stream temperature dynamics: Measurements and modeling. Water Resources Research 29, 2299-2312. https://doi.org/10.1029/93WR00540

Stefan, H.G., Preud'homme, E.B., 1993. Stream Temperature Estimation from Air Temperature. JAWRA Journal of the American Water Resources Association 29, 27-45. https://doi.org/10.1111/j.1752-1688.1993.tb01502.x

Story, A., Moore, R.D., Macdonald, J.S., 2003. Stream temperatures in two shaded reaches below cutblocks and logging roads: downstream cooling linked to subsurface hydrology. Canadian Journal of Forest Research 33, 1383-1396. https://doi.org/10.1139/x03-087

Sun, N., Yearsley, J., Voisin, N., Lettenmaier, D.P., 2015. A spatially distributed model for the assessment of land use impacts on stream temperature in small urban watersheds. Hydrological Processes 29, 2331-2345. https://doi.org/10.1002/hyp.10363

Tetzlaff, D., Carey, S.K., McNamara, J.P., Laudon, H., Soulsby, C., 2017. The essential value of long-term experimental data for hydrology and water management: LONG-TERM DATA IN HYDROLOGY. Water Resour. Res. 53, 2598-2604. https://doi.org/10.1002/2017WR020838

Todd Petty, J., Hansbarger, J.L., Huntsman, B.M., Mazik, P.M., 2012. Brook trout movement in response to temperature, flow, and thermal refugia within a complex Appalachian riverscape. Transactions of the American Fisheries Society 141, 1060-1073. https://doi.org/10.1080/00028487.2012.681102

Torgersen, C.E., Price, D.M., Li, H.W., McIntosh, B.A., 1999. Multiscale thermal refugia and stream habitat associations of Chinook Salmon is Northeastern Oregon. Ecological 
Applications $\quad 9, \quad 301-319 . \quad$ https://doi.org/10.1890/10510761(1999)009[0301:MTRASH]2.0.CO;2

Trego, C.T., Merriam, E.R., Petty, J.T., 2019. Non-native trout limit native brook trout access to space and thermal refugia in a restored large-river system. Restoration Ecology 27, 892900. https://doi.org/10.1111/rec.12925

U.S. Department of Agriculture, 1997. Usual Planting and Harvesting Dates for U.S. Field Crops. Agric. Handb. 51.

Walsh, C.J., Roy, A.H., Feminella, J.W., Cottingham, P.D., Groffman, P.M., Morgan, R.P., 2005. The urban stream syndrome: current knowledge and the search for a cure. Journal of the North American Benthological Society 24, 706-723. https://doi.org/10.1899/04-028.1

Ward, J. V., 1985. Thermal characteristics of running waters. Hydrobiologia 125, 31-46. https://doi.org/10.1007/BF00045924

Webb, B W. Nobilis, F., 1997. Long-term perspective on the nature of the water-air temperature relationship - a case study. Hydrological Processes 11, 137-147.

Webb, B.W., 1996. TRENDS IN STREAM A N D RIVER TEMPERATURE 10, 205-226.

Webb, B.W., Hannah, D.M., Moore, R.D., Brown, L.E., Nobilis, F., 2008. Recent advances in stream and river temperature research 918, 902-918. https://doi.org/10.1002/hyp

Webb, B.W., Y, Z., 1997. Spatial and Seasonal Variablility in The Componeets of The River Heat Budget. Hydrological Processes 11, 79-101.

Webb, B.W., Zhang, Y., 1999. Water temperatures and heat budgets in Dorset chalk water courses 321, 309-321.

Wei, L., Hubbart, J.A., Zhou, H., 2018. Variable Streamflow Contributions in Nested Subwatersheds of a US Midwestern Urban Watershed. Water Resources Management 32, 213-228. https://doi.org/10.1007/s11269-017-1804-5

Wiebe, K.D., Gollehon, N.R., 2007. Agricultural Resources and Environmental Indicators. Nova Publishers.

Winder, M., Sommer, U., 2012. Phytoplankton response to a changing climate. Hydrobiologia 698, 5-16. https://doi.org/10.1007/s10750-012-1149-2

Xuhui, L., 2018. Fundamentals of Boundary- Layer Meteorology. 
Younus, M., Hondzo, M., Engel, B.A., 2000. Stream Temperature Dynamics in Upland Agricultural Watersheds. J. Environ. Eng. 126, 518-526. https://doi.org/10.1061/(ASCE)0733-9372(2000)126:6(518)

Zajíček, A., Kvítek, T., Kaplická, M., Doležal, F., Kulhavý, Z., Bystřický, V., Žlábek, P., 2011. Drainage water temperature as a basis for verifying drainage runoff composition on slopes. Hydrological Processes 25, 3204-3215. https://doi.org/10.1002/hyp.8039

Zeiger, S., Hubbart, J.A., Anderson, S.H., Stambaugh, M.C., 2016. Quantifying and modelling urban stream temperature: a central US watershed study 514, 503-514. https://doi.org/10.1002/hyp.10617

Zeiger, S.J., Hubbart, J.A., 2015. Urban Stormwater Temperature Surges: A Central US Watershed Study 193-209. https://doi.org/10.3390/hydrology2040193 


\section{CHAPTER II \\ EXPLORATORY WORK}

\section{Thermal Surges}

Given the impact that maximum Tw can have on stream ecosystems, a preliminary investigation of thermal surge events was conducted. A relationship between impervious surface cover and stream water temperature surges has been shown in past studies often associated with urban stream syndrome (Anderson et al., 2010; Caldwell et al., 2015; Ficklin et al., 2012; Herb et al., 2008a; Herb and Stefan, 2011; Palmer and Nelson, 2007a; Ren et al., 2003; Rice et al., 2011; Segura et al., 2015; Sun et al., 2015; Webb et al., 2008; Wei et al., 2018; Zeiger and Hubbart, 2015). This is important due to the increasing sprawl of mixed development LULCs in West Run Watershed, potentially increasing the symptoms of urban stream syndrome in the future.

Arbitrarily selected precipitation events were targeted to analyze the effects of precipitation events on stream Tw surges. As per Zeiger and Hubbart (2015), Rice et al. (2011), and Anderson et al. (2010), a Tw surge is defined as higher than $1.0{ }^{\circ} \mathrm{C}$ increase within 15 -min time interval. In Zeiger and Hubbart (2015), surge duration was calculated as the time from the beginning of the Tw surge until Tw returned to pre-surge temperature. For the current work, during each event, surge duration, maximum Tw increase, surge lag time, rate of Tw increase, and total precipitation were measured. Replicating the work of Zeiger and Hubbart, (2015) a stacked comparison of $\mathrm{Tw}$ on the $\mathrm{y}$-axis and time on the $\mathrm{x}$-axis will be created to show the lag time from precipitation event to observed temperature surge upon entrance, and the thermal plume created as the warmer water enters the watershed and moves through the watershed (Figure 7).

$\mathrm{H}_{0}$ : Storm surges will not be detected following precipitation events in West Run Watershed

$\mathrm{H}_{\mathrm{a}}$ : Storm surges will be detected following precipitation events in West Run Watershed

Five thermal surge events were identified, randomly chosen, and isolated from the 2018 Tw annual data by looking for a $1.0 \mathrm{C}^{\circ}$ increase within a $15 \mathrm{~min}$ period following summer precipitation events. Between all five precipitation events mean precipitation, Tw surge, surge 
duration, rate of $\mathrm{Tw}$ increase, and lag time were $14.26 \mathrm{~mm}, 2.46{ }^{\circ} \mathrm{C}, 4.64$ hours, $0.1{ }^{\circ} \mathrm{C}$, and 8 mins, respectively (Table 6). The largest precipitation event occurred on July $31^{\text {st }}$ at 14:05
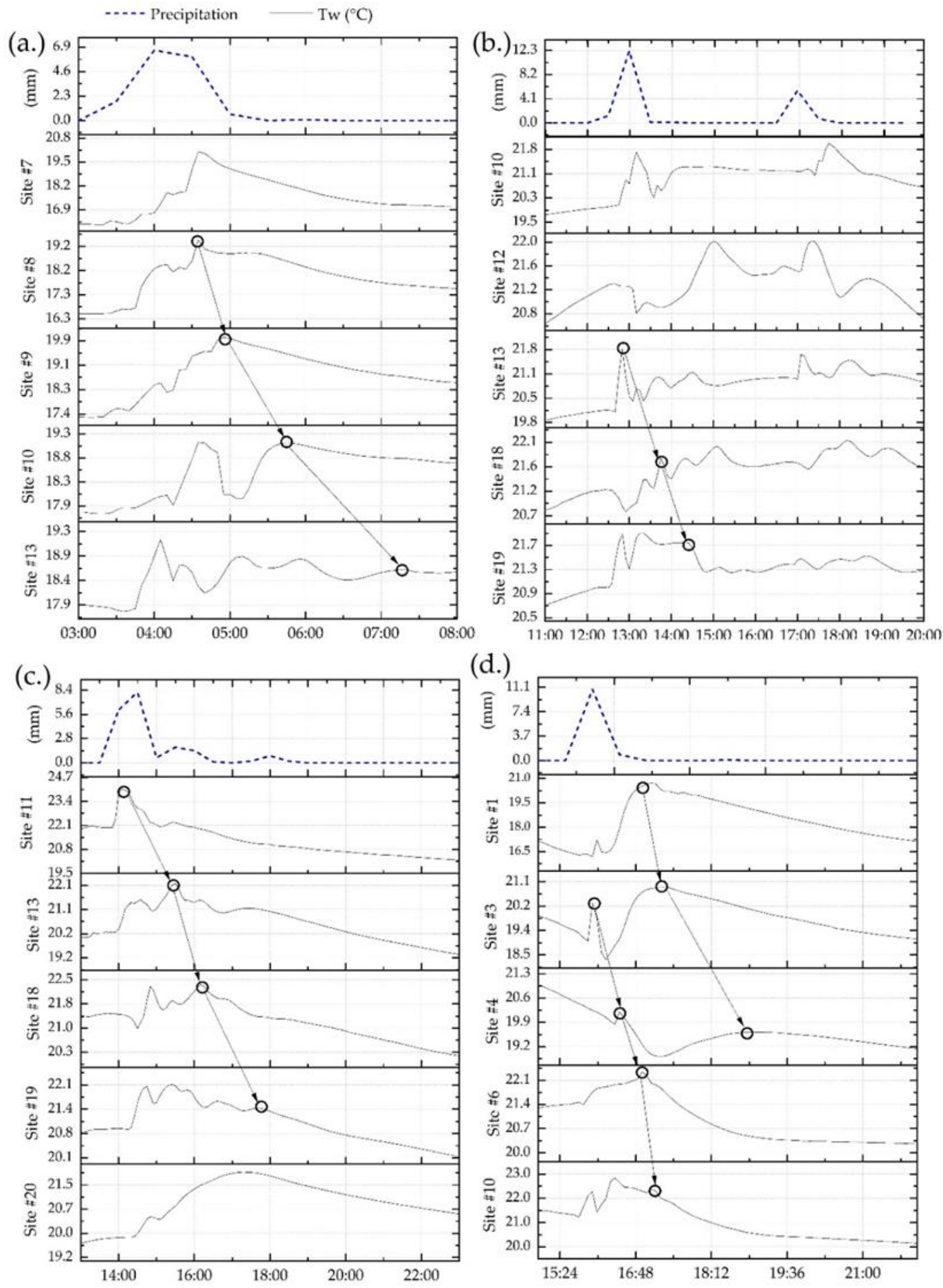

Figure 7. Stream water temperature surges sensed at sites in West Run Creek following summer precipitation event during the summer of 2018. Black circles mark the peak Tw surges and 
arrows track the Tw surge as it moves downstream. (a) Stream water temperature surge one occurring on 06/17 at 04:15 (15.1mm precipitation event) moving through West Run Creek. (b) Stream water temperature surge two occurring on 07/25 at 12:00 (13.5mm precipitation event) moving through West Run Creek. (c) Stream water temperature surge three occurring on 07/16 at 13:00 (19.4mm precipitation event) moving through West Run Creek. (d) Stream water temperature surge four (left) occurring on 08/16 at 15:55 (11.7mm precipitation event) and stream water temperature surge five (right) occurring on 08/16 at 16:15 (11.7mm precipitation).

Table 6. Descriptive statistics of stream water temperature surges identified during Quarter 3 of the 2018 annual water temperature data collected at West Run Watershed, West Virginia, USA.

\begin{tabular}{lcccccc}
\hline & Date (Time) & $\begin{array}{c}\text { Total } \\
\text { Precipitation } \\
(\mathbf{m m})\end{array}$ & $\begin{array}{c}\text { Surge } \\
\text { Amplitude }\left({ }^{\circ} \mathbf{C}\right)\end{array}$ & $\begin{array}{c}\text { Rate of } \\
\text { increase } \\
\left({ }^{\circ} \mathbf{C} / \mathbf{m i n}\right)\end{array}$ & $\begin{array}{c}\text { Lag Time } \\
(\text { mins })\end{array}$ & $\begin{array}{c}\text { Surge } \\
\text { Duration } \\
(\text { hours })\end{array}$ \\
\hline Surge \#1 & $6 / 17(4: 15)$ & 15.1 & 2.8 & 0.1 & 10 & 1.8 \\
Surge \#2 & $7 / 25(12: 40)$ & 13.6 & 1.7 & 0.1 & 10 & 10.6 \\
Surge \#3 & $7 / 31(14: 05)$ & 19.4 & 2.1 & 0.1 & 5 & 2.5 \\
Surge \#4 & $8 / 16(15: 55)$ & 11.7 & 1.4 & 0.1 & 0 & 0.3 \\
Surge \#5 & $8 / 16(16: 15)$ & 11.7 & 4.3 & 0.1 & 15 & 8 \\
\hline
\end{tabular}

at Site \#11, with a total of $19.4 \mathrm{~mm}$ of precipitation falling over 4.5 hours, resulting in a surge increasing Tw by $2.1{ }^{\circ} \mathrm{C}$ lasting 2.5 hours (Figure 7). The largest Tw surge increase of $4.3{ }^{\circ} \mathrm{C}$ occurred on August $16^{\text {th }}$ at $16: 15$ at Site \#1, dominate LULC mixed development $\left(0.1 \mathrm{~km}^{2}\right)$ with a total of $11.7 \mathrm{~mm}$ of precipitation falling over 1.0 hour, producing a surge lasting 8 hours. Two thermal surges were observed during the August $16^{\text {th }}$ event, both observed at Site \#3, dominate LULC Forested $\left(1.1 \mathrm{~km}^{2}\right)$. The longest surge duration occurred on July $25^{\text {th }}$ at $12: 40$ at Site \#13, dominate LULC Forested $\left(5.0 \mathrm{~km}^{2}\right)$ with a total of $13.6 \mathrm{~mm}$ of precipitation falling over 2 hours, resulting in a surge increase Tw by $1.7^{\circ} \mathrm{C}$ lasting 10.6 hours.

The analysis of temperature surges was limited in this study. A rating curve for each site to estimate flow is needed for further investigation. However, our hypothesis was rejected when locating and isolating these five events. Other multiple surges were identified but left for quantitation and analysis in future research. Furthermore, multiple surge events occurring simultaneously in the watershed were observed and only able to be identified because of the large number of sampling sites. The topic of multiple surges occurring simultaneously has been 
studied minimally in past literature and needs to be investigated. This investigation showed thermal surges in the study watershed, and therefore presents future research opportunities into the investigation of thermal surge dynamics. Furthermore, the potential correlation between summer thermal surge events and agricultural LULC types should be investigated. The relationship between mixed-development, particularly impervious surfaces, frequently appears in the Tw literature. However, the relationship to agricultural LULC types is not well understood. Future studies analyzing thermal surges in WRW could potentially advance the decision-making success of land managers and policy makers concerned with the health of aquatic ecosystems.

\section{Relationship of Stream Distance and Tw}

Ambient air temperature (Ta) has been shown to have a substantial influence on $\mathrm{Tw}$ (O’Driscoll and DeWalle, 2006; Rice et al., 2011; Sinokrot and Stefan, 1993). Research has shown conclusively that, as water moves downstream, it is impacted less by the various inflows from runoff/tributaries, and more so by ambient air temperature (Ta) (O'Driscoll and DeWalle, 2006; Sinokrot and Stefan, 1993). However, it should also be stated that land-use also influences Ta in addition to Tw (Moore et al., 2005). Rice et al. (2011) showed that increasing watershed urbanization causes the Tw and Ta relationship to break down. Tw begins to deviate from Ta as energy and mass accumulate from surrounding mixed development LULC types. This is, in some instances, offset given that higher channel volumes downstream are often sufficient enough to attenuate thermal inputs (Caissie, 2006; Gu et al., 1999). Thus, stream inputs (e.g., groundwater, surface runoff, tributaries) from surrounding land-use types may have a greater influence upstream near headwaters relative to downstream and during periods of low(er) flow relative to periods of flooding (Webb et al. 2008).

A linear relationship between stream distance and descriptive statistics from sites along the main channel was fit using simple linear regression to validate results from past research were stream distance was showed to have a significant correlation with Tw variables (e.g., mean, minimum, maximum, and standard deviation) (Anderson et al., 2010; Mohseni and Stefan, 1999; Poole and Berman, 2001; Stefan and Preud'homme, 1993). Ta recorded at a climate station near site \#13 was assumed homogenous across the entire watershed.

$\mathrm{H}_{0}$ : There will be no significant relationship between distance from headwaters and $\mathrm{Tw}$ variables (mean, maximum, minimum, standard deviation) 
$\mathrm{H}_{\mathrm{a}}$ : There will be a significant relationship between distance from headwaters and $\mathrm{Tw}$ variables (mean, maximum, minimum, standard deviation)

The linear relationship fit between mean $(\mathrm{p}=0.04)$, maximum $(\mathrm{p}=0.03)$, and standard deviation $(\mathrm{p}=0.01$ ) showed significance at an $\alpha=0.05$ to stream distance from the headwaters of West Run Creek, similar to studies looking at relations between stream distance downstream and Tw (Figure 8) (Anderson et al., 2010; Mohseni and Stefan, 1999; Poole and Berman, 2001; Stefan and Preud'homme, 1993; Torgersen et al., 1999) In all three cases, the relationship had a positive correlation and R-squared values of $0.68,0.57$, and 0.71 , respectively. Minimum Tw did not have a significant relationship $(\mathrm{p}=0.90)$ to distance downstream from headwater.
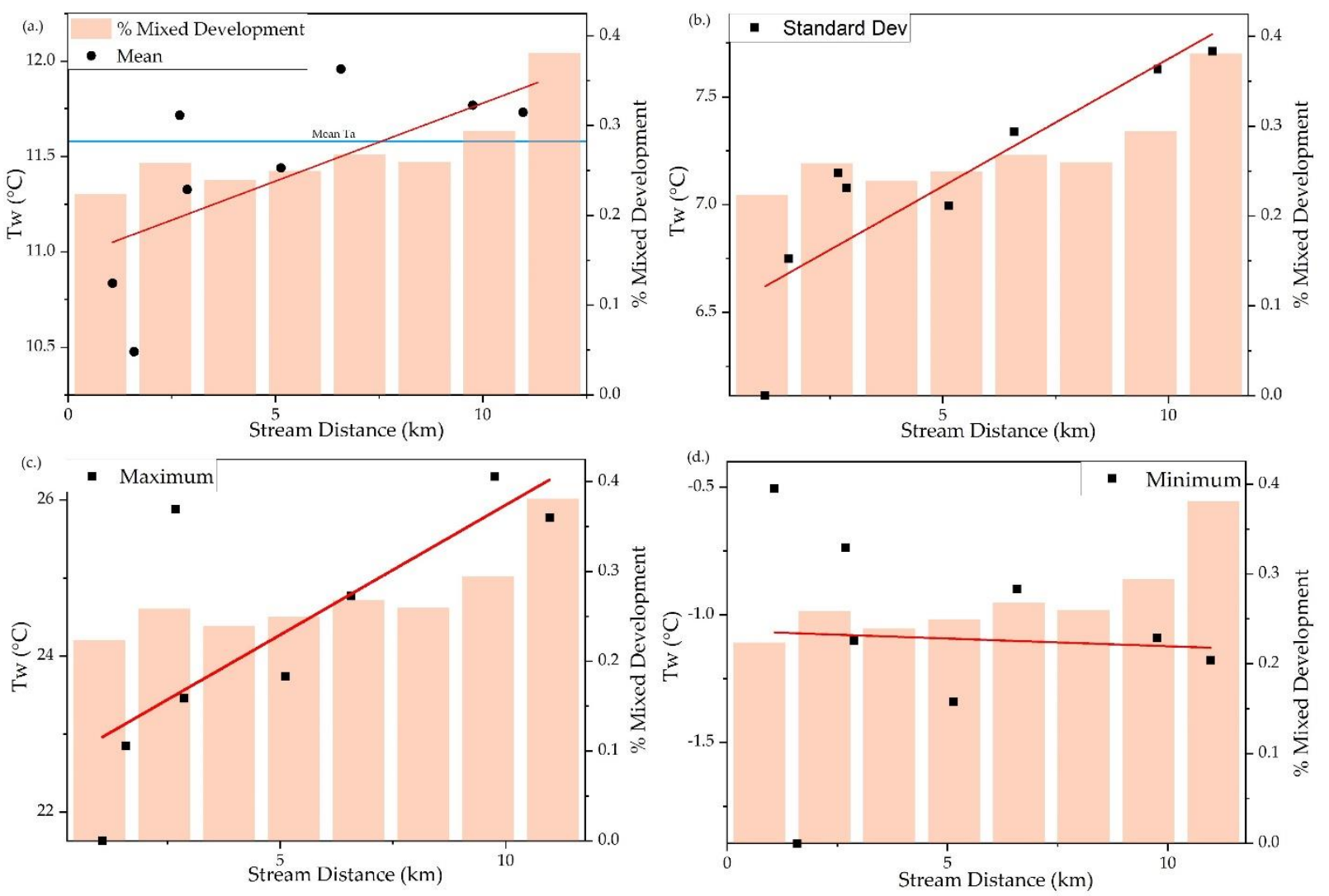

Figure 8. Mean (a), standard deviation (b), maximum (c), and minimum (d) water temperature collected at main stem gauging sites of West Run Watershed, West Virginia, USA; plotted against stream distance from headwaters of West Run Creek. Percent mixed development LULC is also plotted against stream distance from headwaters. A reference line for mean Ta over the entire 2018 annual year is included in mean plot (a). 
Given these results the null hypothesis was rejected. These results are important because they align with the conclusions of past researchers that as parcels of water move downstream, Tw tends to increase (Anderson et al., 2010; Bogan et al., 2003; Herb et al., 2008; Palmer and Nelson, 2007; Rice et al., 2011). This increase is attributed to equilibration with atmospheric conditions and/or compounding effects of urbanization (Figure 8) (Anderson et al., 2010; Rice et al., 2011; Zeiger and Hubbart, 2015). Overlying Ta affects rates of sensible heat transfer to or from adjacent streams depending on initial temperature deficits (Bogan et al., 2003). These temperature deficits between Ta and Tw occur due to the atmosphere's lower heat capacity relative to liquid water (Bogan et al., 2003; Mohseni and Stefan, 1999; Poole and Berman, 2001). Furthermore, stream water further downstream tends to have higher thermal inertia due to larger volumetric streamflow and is less affected by incoming surface runoff, tributary inflows, overlying Ta, and groundwater inputs (Johnson, M. Wilby, 2007; Mwedzi et al., 2016; Poole and Berman, 2001; Rice et al., 2011; Sinokrot and Gulliver, 2000). This higher thermal inertia means surrounding land-use types should have a smaller effect on Tw. However, Rice et al. (2011) observed the compounding effect of urban areas, associated with the mixed development LULC types increase Tw prevailing over dampening effects caused by higher thermal inertia. Furthermore, downstream sub-basins of West Run Watershed have lower portions of forested LULC areas and greater mixed development and agricultural LULCs (Figure 2.) an additional reason explaining why both mean, maximum, and standard deviation of Tw increases with distance downstream (Rice et al., 2011).

\section{Future Work}

Given the finding of thermal surge events occurring in West Run Watershed these thermal surge events should be further investigated. Future research should be conducted to quantify all surge events over not only the 2018 annual year but also 2017 and 2019 annual years for which Tw data exists. The Incorporation of two additional annual year passes the three-year threshold needed to classify the research investigate as an investigation of West Run Watershed's thermal regime. The three years of data should elucidate the interactions occurring between surrounding LULC types and thermal surge dynamics (e.g., surge amplitude, surge frequency, surge duration). The 2017 and 2019 annual years were not incorporated into this investigation due to ongoing 2019 data collection during the investigation and lack of post processing of 2017 data. As stated, surges were detected at not just a single location in the 
watershed, but multiple locations throughout the watershed during the same precipitation event. In the current literature, there is a lack of information regarding these multi-surge events. The current literature attributes surge events to urban area and impervious surfaces associated with mixed-development LULC types (Anderson et al., 2010; Rice et al., 2011; Zeiger and Hubbart, 2015). Given the significant relation between maximum water temperature and agricultural LULC types, the potential impact of agriculture LULCs on thermal surge dynamics should also be investigated in for future research. Furthermore, the creation of a thermal surge model in West Run Watershed to predict surge likelihood and severity would be a good avenue for future research and be a beneficial tool for land managers. Climatic conditions prior to precipitation events like incident shortwave radiation have been shown to be an influencing factor on surge occurrence and severity (i.e., surge amplitude and surge duration). Creating a model which incorporates variables such as shortwave radiation flux, percent impervious surfaces, amount of precipitation, spatial precipitation variability, and topographic slope may allow researcher to predict thermal surge likelihood and severity.

The high-resolution data acquired via direct measurements of Tw in West Run Watershed could be used to validate the numerous existing Tw models. Numerous Tw models have been developed to predict Tw. For example, effective models in predicting Tw exist which use only overlying air temperature (Mohseni and Stefan, 1999; Stefan and Preud'homme, 1993). Other models such as the SNTEMP model or SWAT model incorporate other variables (e.g., streamflow, ground water temperature, hyporheic water temperature, and net heat exchange by radiation, turbulent exchange, and conduction across the water surface and bed) (Herb et al., 2008; Moore et al., 2005). The validation and further development of both simple and complex existing models provides another potential avenue for scientific advances and future research. 


\section{Literature Cited}

Anderson, W.P., Anderson, J.L., Thaxton, C.S., Babyak, C.M., 2010. Changes in stream temperatures in response to restoration of groundwater discharge and solar heating in a culverted, urban stream. Journal of Hydrology 393, 309-320. https://doi.org/10.1016/j.jhydrol.2010.08.030

Arismendi, I., Safeeq, M., Dunham, J.B., Johnson, S.L., 2014. Can air temperature be used to project influences of climate change on stream temperature? Environmental Research Letters 9. https://doi.org/10.1088/1748-9326/9/8/084015

Beitinger, T.L., Bennett, W.A., Mccauley, R.W., 2000. Temperature tolerances of North American freshwater fishes exposed to dynamic changes in temperature. Environmental Biology of Fishes 58, 237-275.

Beschta, R.L., Taylor, R.L., 1988. Stream Temperature Increases and Land Use in a Forested Oregon Watershed. JAWRA Journal of the American Water Resources Association 24, 19-25. https://doi.org/10.1111/j.1752-1688.1988.tb00875.x

Bogan, T., Mohseni, O., Stefan, H.G., 2003. Stream temperature-equilibrium temperature relationship. Water Resources Research 39, 1-12. https://doi.org/10.1029/2003WR002034

Booth, D.B., Roy, A.H., Smith, B., Capps, K.A., 2016. Global perspectives on the urban stream syndrome. Freshwater Science 35, 412-420. https://doi.org/10.1086/684940

Borman, M., Larson, L., 2003. A case study of river temperature response to agricultural land use and environmental thermal patterns. Journal of soil and water conservation 58, 8-12.

Boyde, C., Drinking, A., Quality, W., Main, W., Program, F., Program, C.C., Program, W.P., 2016. Water Quality an Introduction.

Caissie, D., 2006. The thermal regime of rivers: A review. Freshwater Biology 51, 1389-1406. https://doi.org/10.1111/j.1365-2427.2006.01597.x

Coutant, C.C., 1999. Perspectives on Temperature in the Pacific Northwest's Fresh Waters (No. ORNL/TM-1999/44, 9042). https://doi.org/10.2172/9042

Ficklin, D.L., Luo, Y., Stewart, I.T., Maurer, E.P., 2012. Development and application of a hydroclimatological stream temperature model within the Soil and Water Assessment Tool. Water Resources Research 48, 1-16. https://doi.org/10.1029/2011WR011256 
Gravelle, J.A., Link, T.E., 2007. Influence of timber harvesting on headwater peak stream temperatures in a northern Idaho watershed. (Special issue: Science and management of forest headwater streams). Forest Science 53, 189-205.

Herb, W., Janke, B., Mohseni, O., Stefan, H., 2008. Thermal pollution of streams by runoff from paved surfaces. Hydrological Processes 22, 987-999. https://doi.org/10.1002/hyp

Johnson, S.L., Jones, J.A., 2011. Stream temperature responses to forest harvest and debris flows in western Cascades, Oregon. Canadian Journal of Fisheries and Aquatic Sciences 57, 3039. https://doi.org/10.1139/f00-109

LeBlanc, R.T., Brown, R.D., Fitz Gibbon, J.E., 1997. Modeling the effects of land use change on the water temperature in unregulated urban streams. Journal of Environmental Management 49, 445-469. https://doi.org/10.1006/jema.1996.0106

Ma, Q., Wu, J., He, C., 2016. A hierarchical analysis of the relationship between urban impervious surfaces and land surface temperatures: spatial scale dependence, temporal variations, and bioclimatic modulation. Landscape Ecol 31, 1139-1153. https://doi.org/10.1007/s10980016-0356-z

Martin, R.W., Petty, J.T., 2009. Local stream temperature and drainage network topology interact to influence the distribution of smallmouth bass and brook trout in a Central appalachian watershed. Journal of Freshwater Ecology 24, 497-508. https://doi.org/10.1080/02705060.2009.9664323

Marzadri, A., Tonina, D., Bellin, A., 2013. Effects of stream morphodynamics on hyporheic zone thermal regime. Water Resources Research 49, 2287-2302. https://doi.org/10.1002/wrcr.20199

Mohseni, O., Stefan, H.G., 1999. Stream temperature/air temperature relationship: A physical interpretation. Journal of Hydrology 218, 128-141. https://doi.org/10.1016/S00221694(99)00034-7

Moore, R.D., Spittlehouse, D.L., Story, A., 2005. Riparian microclimate and stream temperature response to forest harvesting: a review. Journal of the American Water Resources Association 7, 813-834.

Mustafa, M., Barnhart, B., Babbar-Sebens, M., Ficklin, D., 2018. Modeling landscape change effects on stream temperature using the Soil and Water Assessment Tool. Water (Switzerland) 10, 1-18. https://doi.org/10.3390/w10091143 
Naiman, R., Décamps, H., 1997. The ecology of interfaces: Riparian Zones. Annual Review of Ecology, Evolution, and Systematics 28, 621-658. https://doi.org/10.1146/annurev.ecolsys.28.1.621

O’Driscoll, M.A., DeWalle, D.R., 2006. Stream-air temperature relations to classify streamground water interactions in a karst setting, central Pennsylvania, USA. Journal of Hydrology 329, 140-153. https://doi.org/10.1016/j.jhydrol.2006.02.010

Oke, T.R., 1987. Boundary Layer Climates, Second. ed.

Palmer, M.A., Nelson, K.C., 2007. Stream Temperature Surges under Urbanization. Journal of the American Water Resources Association 43, 440-452. https://doi.org/10.1111/ j.17521688.2007.00034.x

Paul, M.J., Meyer, J.L., 2001. Stream In The Urban Landscape. Annual Review of Ecology and Systematics 32, 333-365. https://doi.org/10.1146/annurev.ecolsys.32.081501.114040

Petersen, F., Hubbart, J.A., 2020. Quantifying Escherichia coli and Suspended Particulate Matter Concentrations in a Mixed-Land Use Appalachian Watershed. Water 12, 532. https://doi.org/10.3390/w12020532

Petersen, F., Hubbart, J.A., Kellner, E., Kutta, E., 2018. Land-use-mediated Escherichia coli concentrations in a contemporary Appalachian watershed. Environmental Earth Sciences 77, 1-13. https://doi.org/10.1007/s12665-018-7948-7

Petty, J.T., Thorne, D., Huntsman, B.M., Mazik, P.M., 2014. The temperature-productivity squeeze: Constraints on brook trout growth along an Appalachian river continuum. Hydrobiologia 727, 151-166. https://doi.org/10.1007/s10750-013-1794-0

Pollock, M.M., Beechie, T.J., Liermann, M., E, R., 2009. Stream Temperature Relationships to Forest Harvest in the Olympic Peninsula ,. Fisheries Science 45, 1-33. https://doi.org/10.1111

Poole, G.C., Berman, C.H., 2001. An ecological perspective on in-stream temperature: Natural heat dynamics and mechanisms of human-caused thermal degradation. Environmental Management 27, 787-802. https://doi.org/10.1007/s002670010188

Rice, J.S., Anderson, Jr., W.P., Thaxton, C.S., 2011. Urbanization influences on stream temperature behavior within low-discharge headwater streams. Hydrological Research Letters 5, 27-31. https://doi.org/10.3178/hrl.5.27 
Segura, C., Caldwell, P., Sun, G., Mcnulty, S., Zhang, Y., 2015. A model to predict stream water temperature across the conterminous USA. Hydrological Processes 29, 2178-2195. https://doi.org/10.1002/hyp.10357

Sinokrot, B.A., Gulliver, J.S., 2000. In-stream flow impact on river water temperatures. Journal of Hydraulic Research 38, 339-349. https://doi.org/10.1080/00221680009498315

Sinokrot, B.A., Stefan, H.G., 1993. Stream temperature dynamics: Measurements and modeling. Water Resources Research 29, 2299-2312. https://doi.org/10.1029/93WR00540

Stefan, H.G., Preud'homme, E.B., 1993. Stream Temperature Estimation from Air Temperature. JAWRA Journal of the American Water Resources Association 29, 27-45. https://doi.org/10.1111/j.1752-1688.1993.tb01502.x

Story, A., Moore, R.D., Macdonald, J.S., 2003. Stream temperatures in two shaded reaches below cutblocks and logging roads: downstream cooling linked to subsurface hydrology. Canadian Journal of Forest Research 33, 1383-1396. https://doi.org/10.1139/x03-087

Sun, N., Yearsley, J., Voisin, N., Lettenmaier, D.P., 2015. A spatially distributed model for the assessment of land use impacts on stream temperature in small urban watersheds. Hydrological Processes 29, 2331-2345. https://doi.org/10.1002/hyp.10363

Todd Petty, J., Hansbarger, J.L., Huntsman, B.M., Mazik, P.M., 2012. Brook trout movement in response to temperature, flow, and thermal refugia within a complex Appalachian riverscape. Transactions of the American Fisheries Society 141, 1060-1073. https://doi.org/10.1080/00028487.2012.681102

Torgersen, C.E., Price, D.M., Li, H.W., McIntosh, B.A., 1999. Multiscale thermal refugia and stream habitat associations of Chinook Salmon is Northeastern Oregon. Ecological Applications $\quad 9, \quad 301-319$. $\quad$ https://doi.org/10.1890/10510761(1999)009[0301:MTRASH]2.0.CO;2

Trego, C.T., Merriam, E.R., Petty, J.T., 2019. Non-native trout limit native brook trout access to space and thermal refugia in a restored large-river system. Restoration Ecology 27, 892900. https://doi.org/10.1111/rec.12925

Walsh, C.J., Roy, A.H., Feminella, J.W., Cottingham, P.D., Groffman, P.M., Morgan, R.P., 2005. The urban stream syndrome: current knowledge and the search for a cure. Journal of the North American Benthological Society 24, 706-723. https://doi.org/10.1899/04-028.1 
Webb, B W. Nobilis, F., 1997. Long-term perspective on the nature of the water-air temperature relationship - a case study. Hydrological Processes 11, 137-147.

Webb, B.W., 1996. Trends in stream water temperature 10, 205-226.

Webb, B.W., Hannah, D.M., Moore, R.D., Brown, L.E., Nobilis, F., 2008. Recent advances in stream and river temperature research 918, 902-918. https://doi.org/10.1002/hyp

Webb, B.W., Y, Z., 1997. Spatial and Seasonal Variablility in The Componeets of The River Heat Budget. Hydrological Processes 11, 79-101.

Webb, B.W., Zhang, Y., 1999. Water temperatures and heat budgets in Dorset chalk water courses 321, 309-321.

Wei, L., Hubbart, J.A., Zhou, H., 2018. Variable Streamflow Contributions in Nested Subwatersheds of a US Midwestern Urban Watershed. Water Resources Management 32, 213-228. https://doi.org/10.1007/s11269-017-1804-5

Xuhui, L., 2018. Fundamentals of Boundary- Layer Meteorology.

Younus, M., Hondzo, M., Engel, B.A., 2000. Stream Temperature Dynamics in Upland Agricultural Watersheds. J. Environ. Eng. 126, 518-526. https://doi.org/10.1061/(ASCE)0733-9372(2000)126:6(518)

Zajíček, A., Kvítek, T., Kaplická, M., Doležal, F., Kulhavý, Z., Bystřický, V., Žlábek, P., 2011. Drainage water temperature as a basis for verifying drainage runoff composition on slopes. Hydrological Processes 25, 3204-3215. https://doi.org/10.1002/hyp.8039

Zeiger, S., Hubbart, J.A., Anderson, S.H., Stambaugh, M.C., 2016. Quantifying and modelling urban stream temperature: a central US watershed study 514, 503-514. https://doi.org/10.1002/hyp.10617

Zeiger, S.J., Hubbart, J.A., 2015. Urban Stormwater Temperature Surges: A Central US Watershed Study 193-209. https://doi.org/10.3390/hydrology2040193 


\section{APPENDIX A \\ CLIMATE DURING STUDY PERIOD}

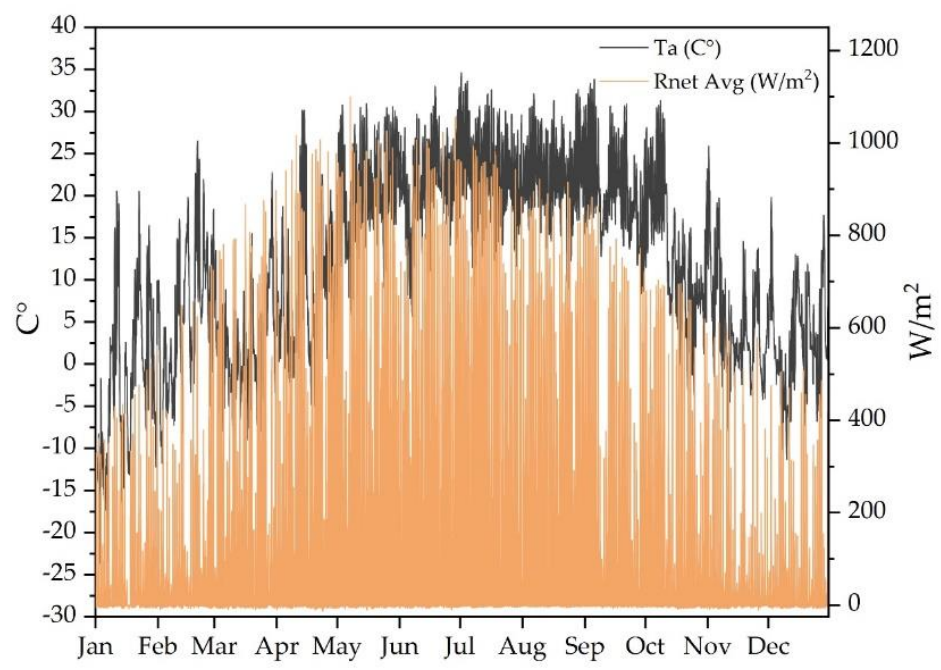

Figure A1. Time-series of ambient air temperature (Ta) and average net radiation across the 2018 annual year collected from a climate station located in West Run Watershed, West Virginia, USA

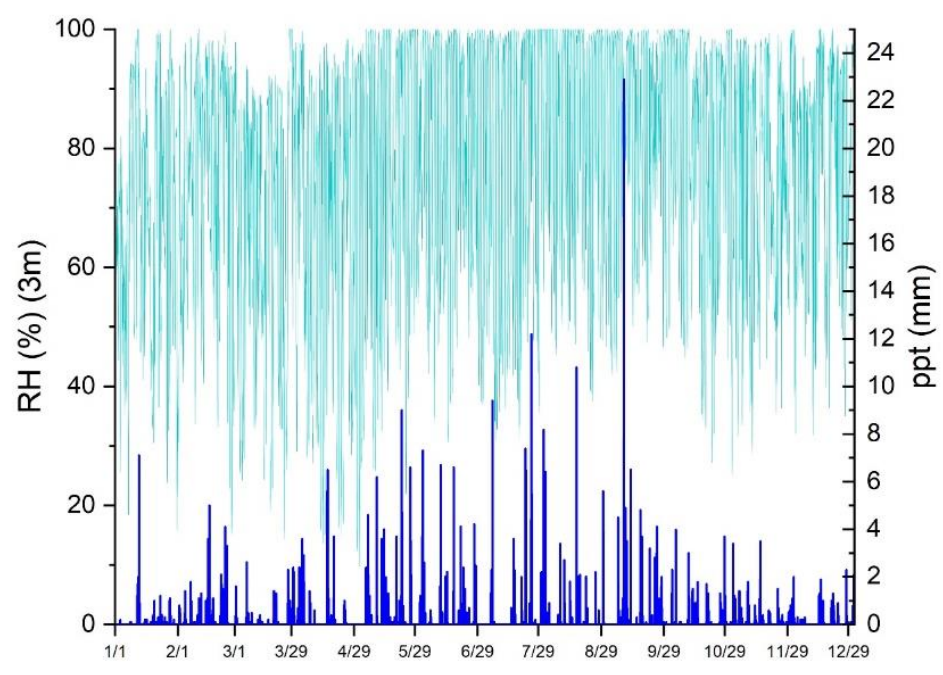

Figure A2. Time-series of relative humidity and precipitation across the 2018 annual year collected from a climate station located in West Run Watershed, West Virginia, USA 


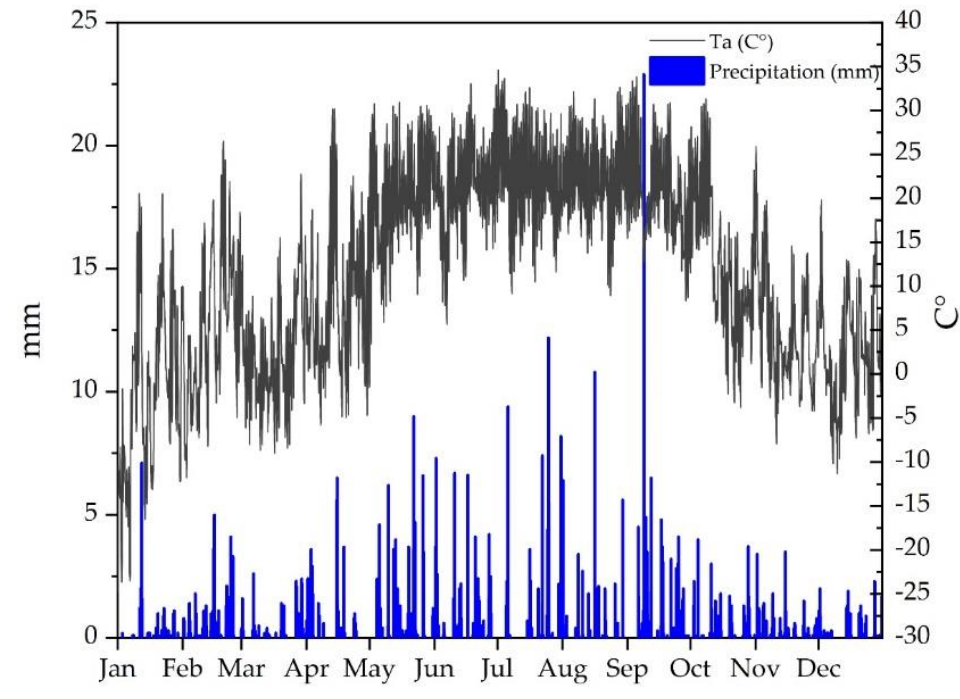

Figure A3. Time-series of ambient air temperature (Ta) and precipitation across the 2018 annual year collected from a climate station located in West Run Watershed, West Virginia, USA 


\section{APPENDIX B \\ GAUGING SITE TW TIMESERIES}

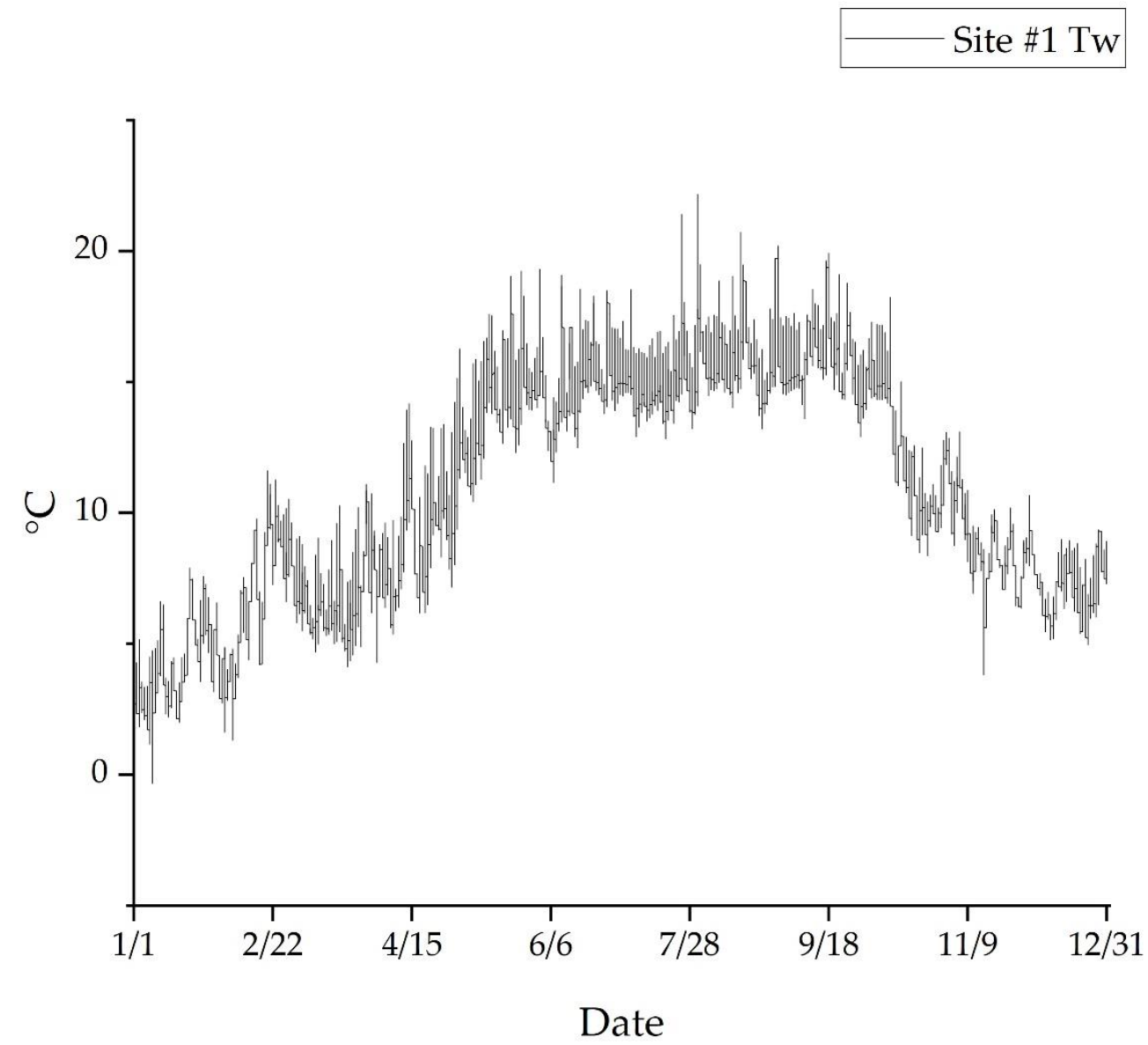

Figure B1. Time-series of steam water temperature across the 2018 annual year recorded at gauging site \#1 located in West Run Watershed, West Virginia, USA. 


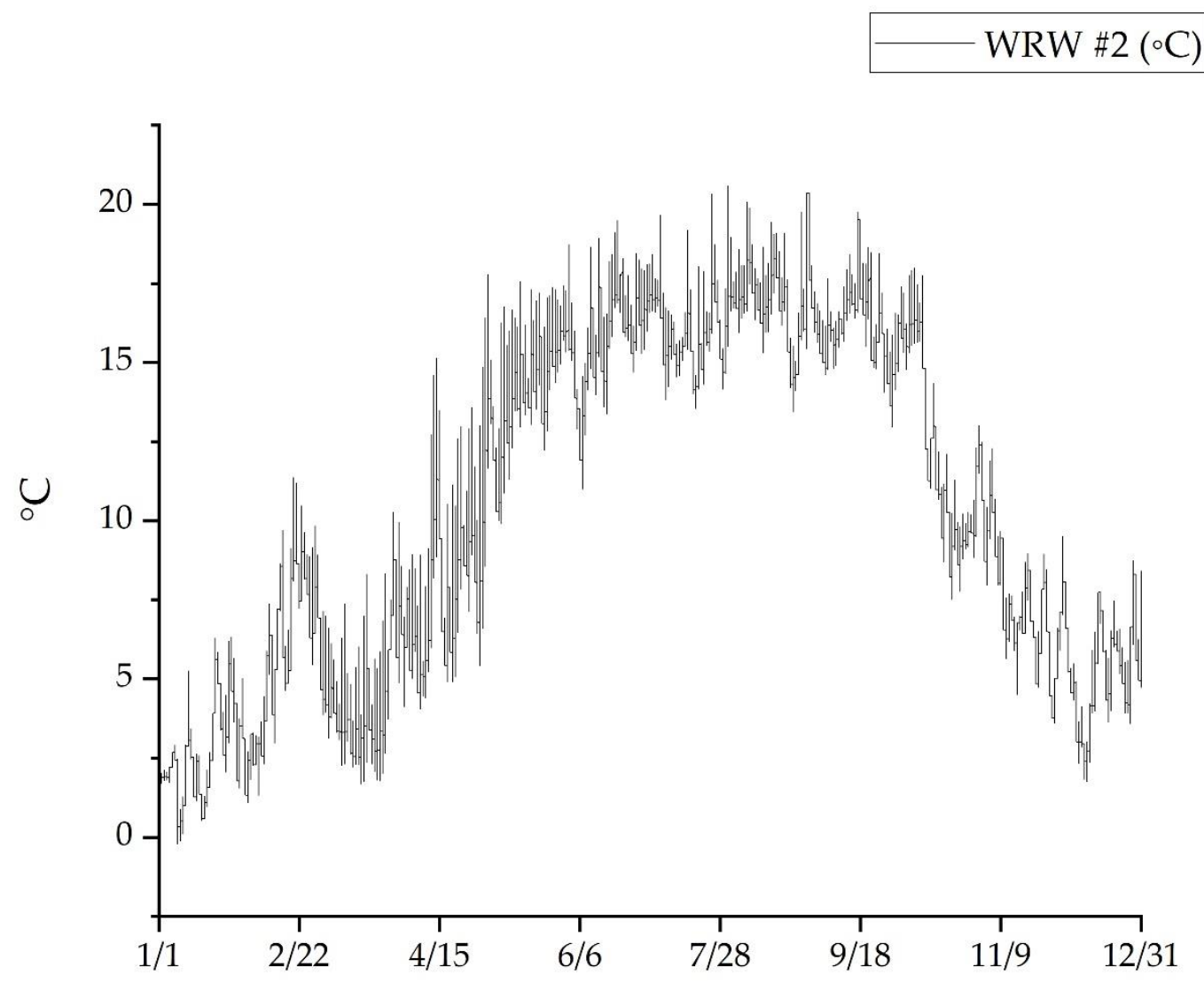

Figure B2. Time-series of steam water temperature across the 2018 annual year recorded at gauging site \#2 located in West Run Watershed, West Virginia, USA. 


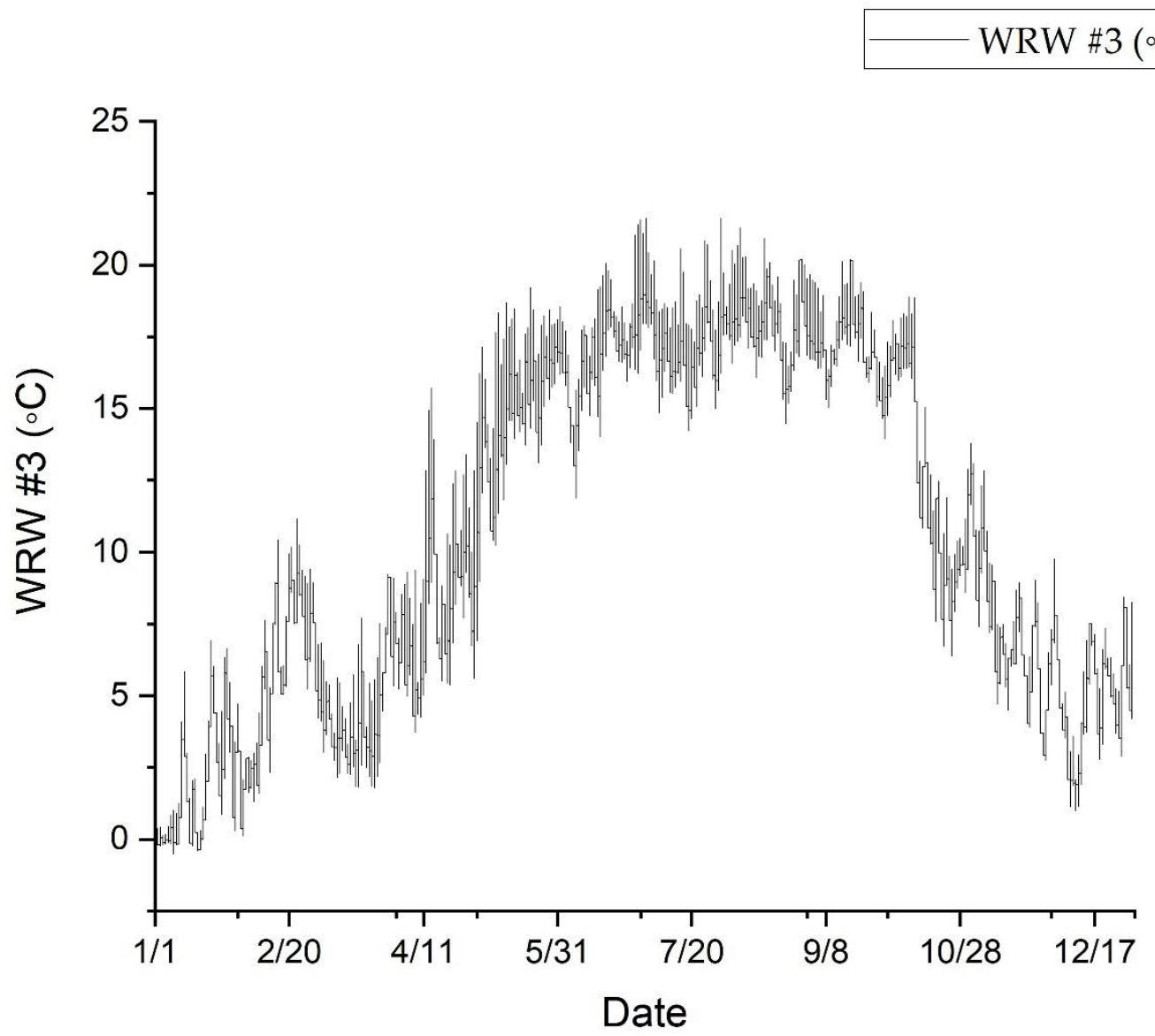

Figure B3. Time-series of steam water temperature across the 2018 annual year recorded at gauging site \#3 located in West Run Watershed, West Virginia, USA. 


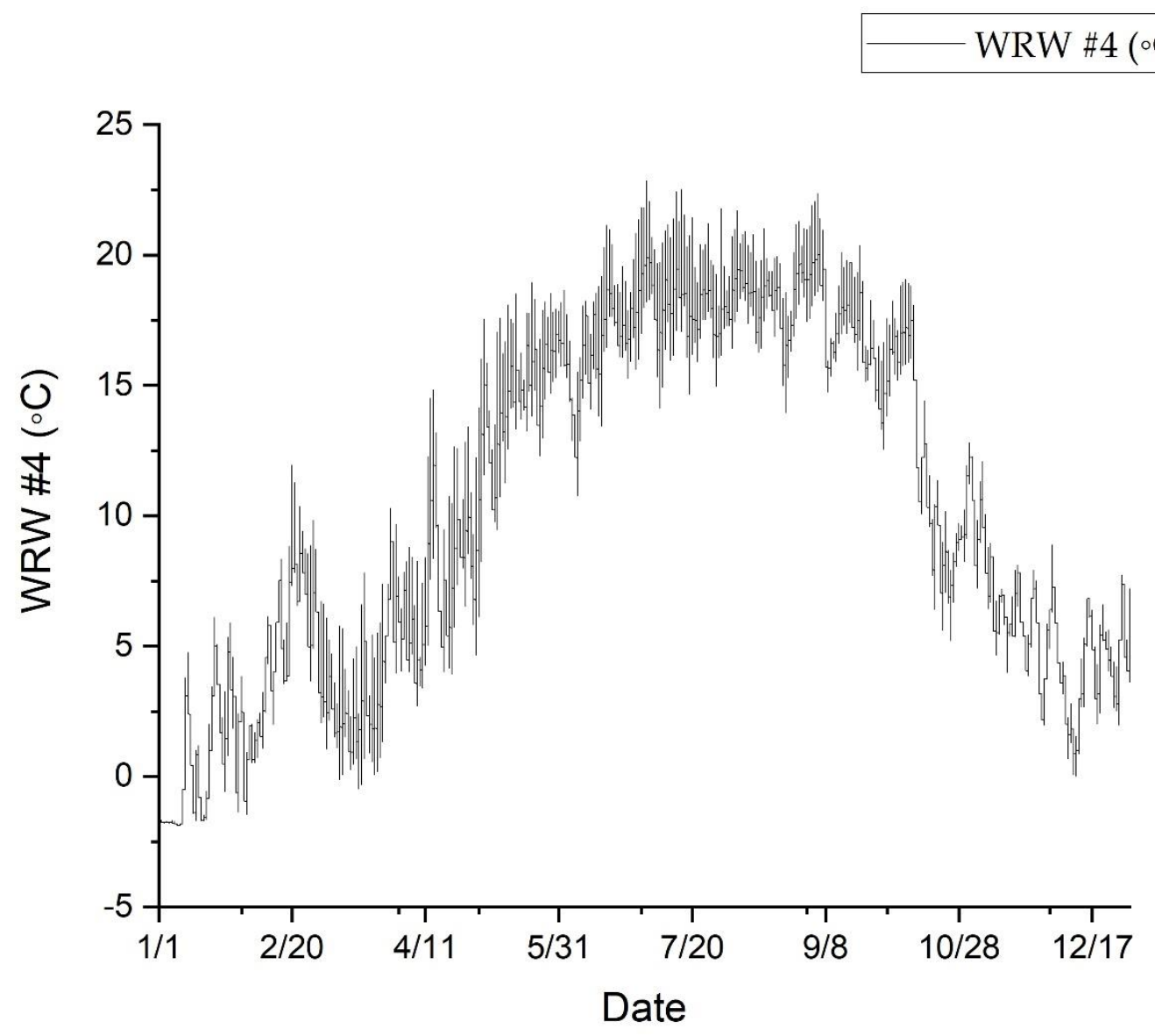

Figure B4. Time-series of steam water temperature across the 2018 annual year recorded at gauging site \#4 located in West Run Watershed, West Virginia, USA. 


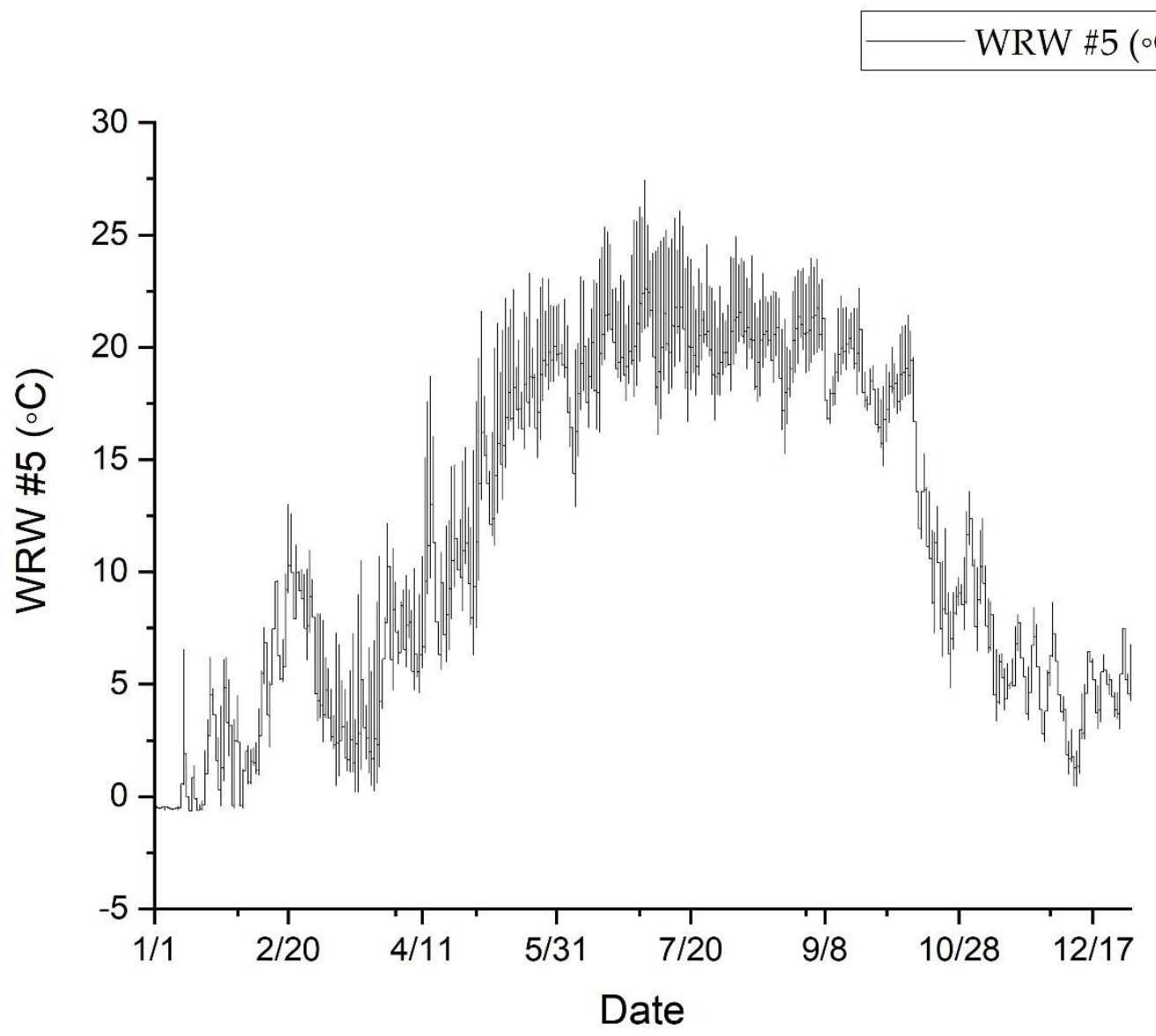

Figure B5. Time-series of steam water temperature across the 2018 annual year recorded at gauging site \#5 located in West Run Watershed, West Virginia, USA. 


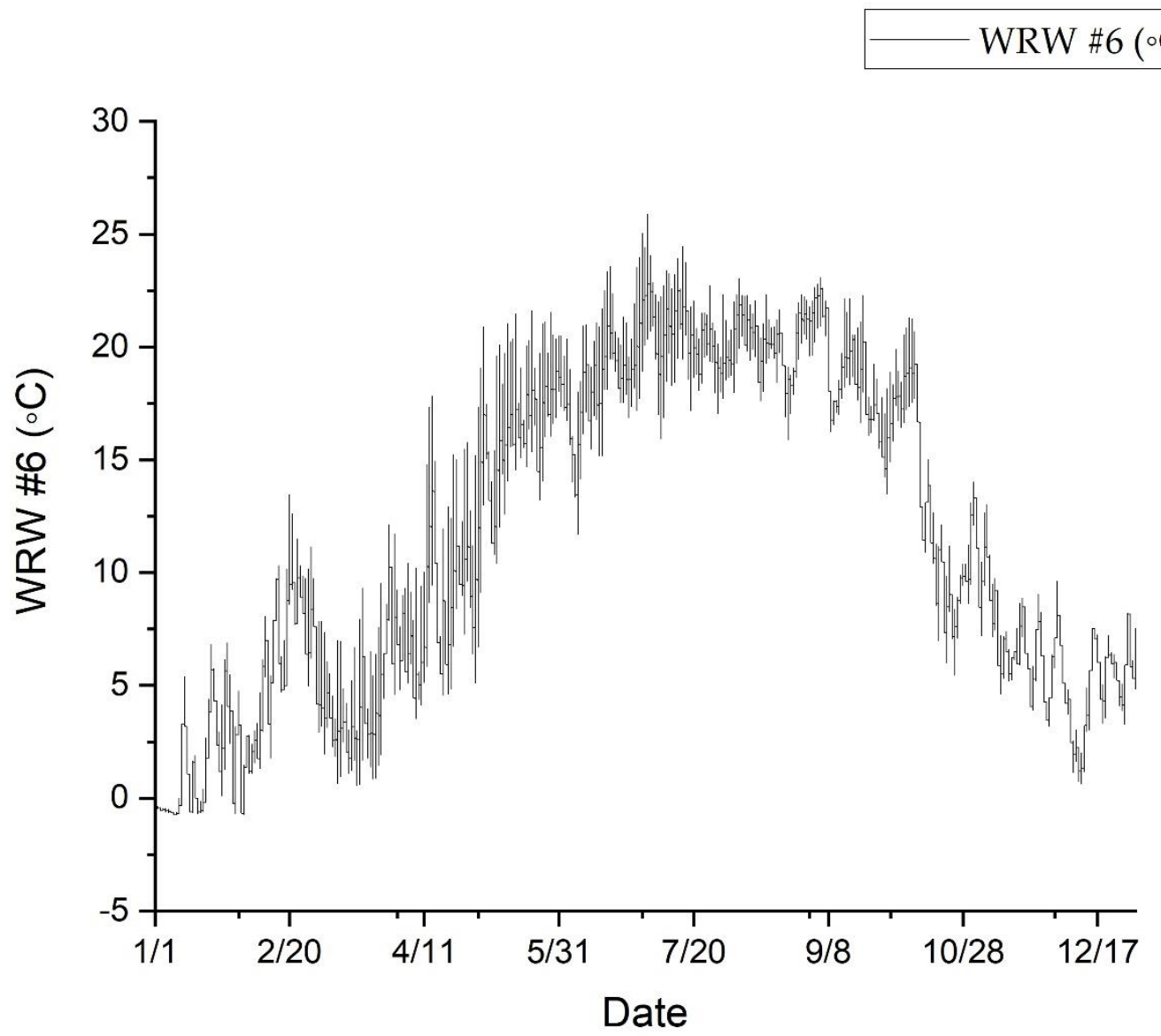

Figure B6. Time-series of steam water temperature across the 2018 annual year recorded at gauging site \#6 located in West Run Watershed, West Virginia, USA. 


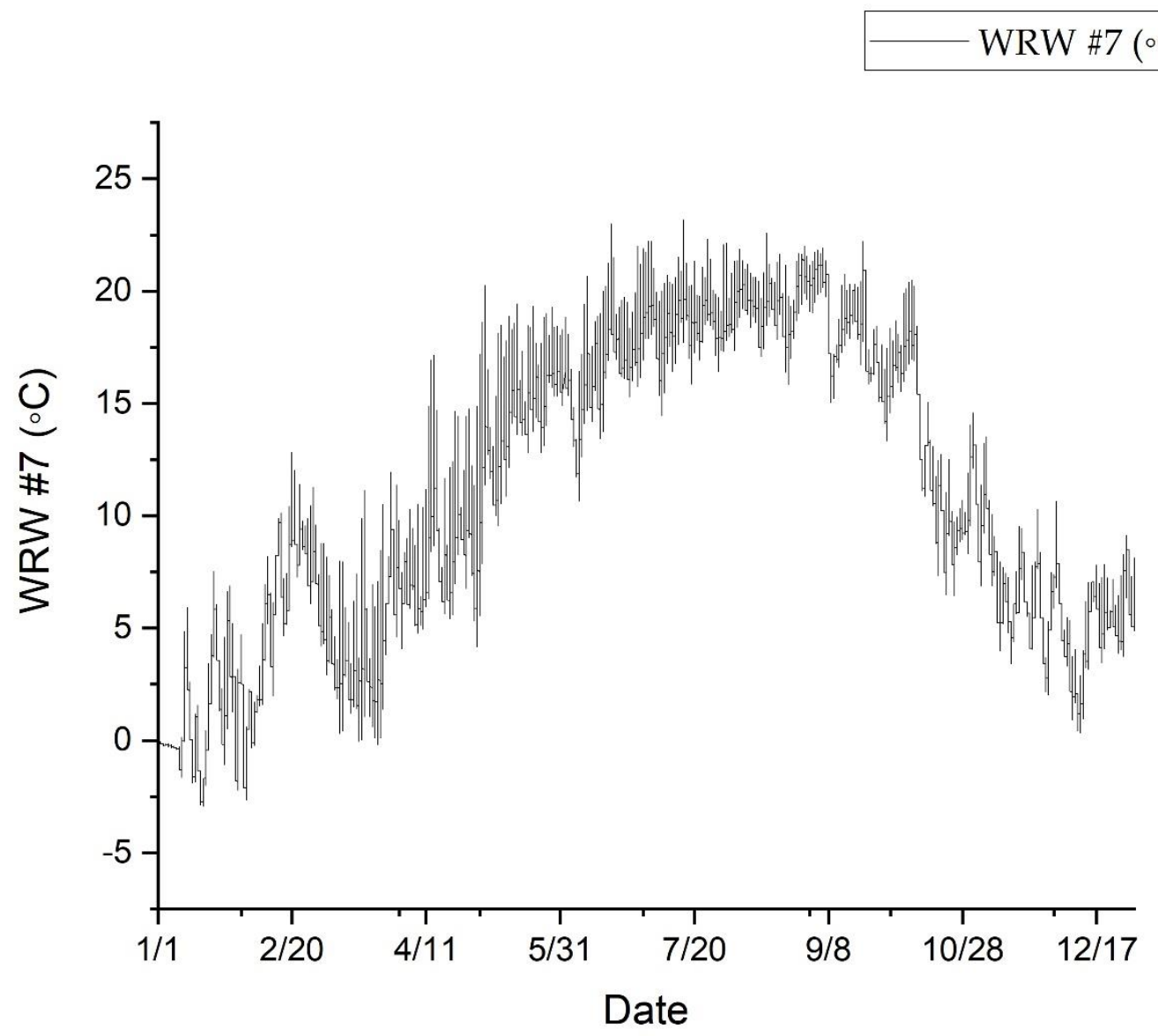

Figure B7. Time-series of steam water temperature across the 2018 annual year recorded at gauging site \#7 located in West Run Watershed, West Virginia, USA. 


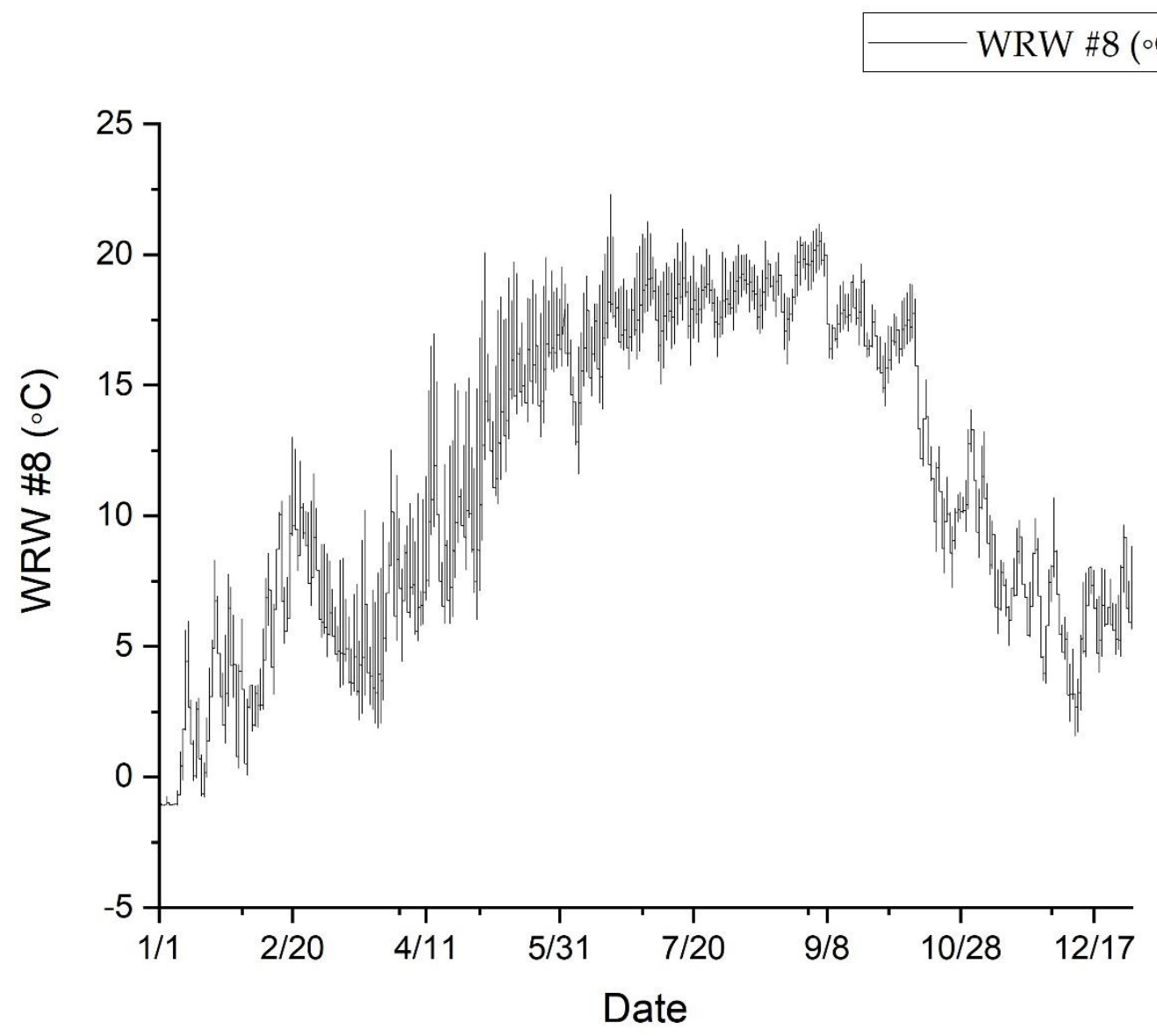

Figure B8. Time-series of steam water temperature across the 2018 annual year recorded at gauging site \#8 located in West Run Watershed, West Virginia, USA. 


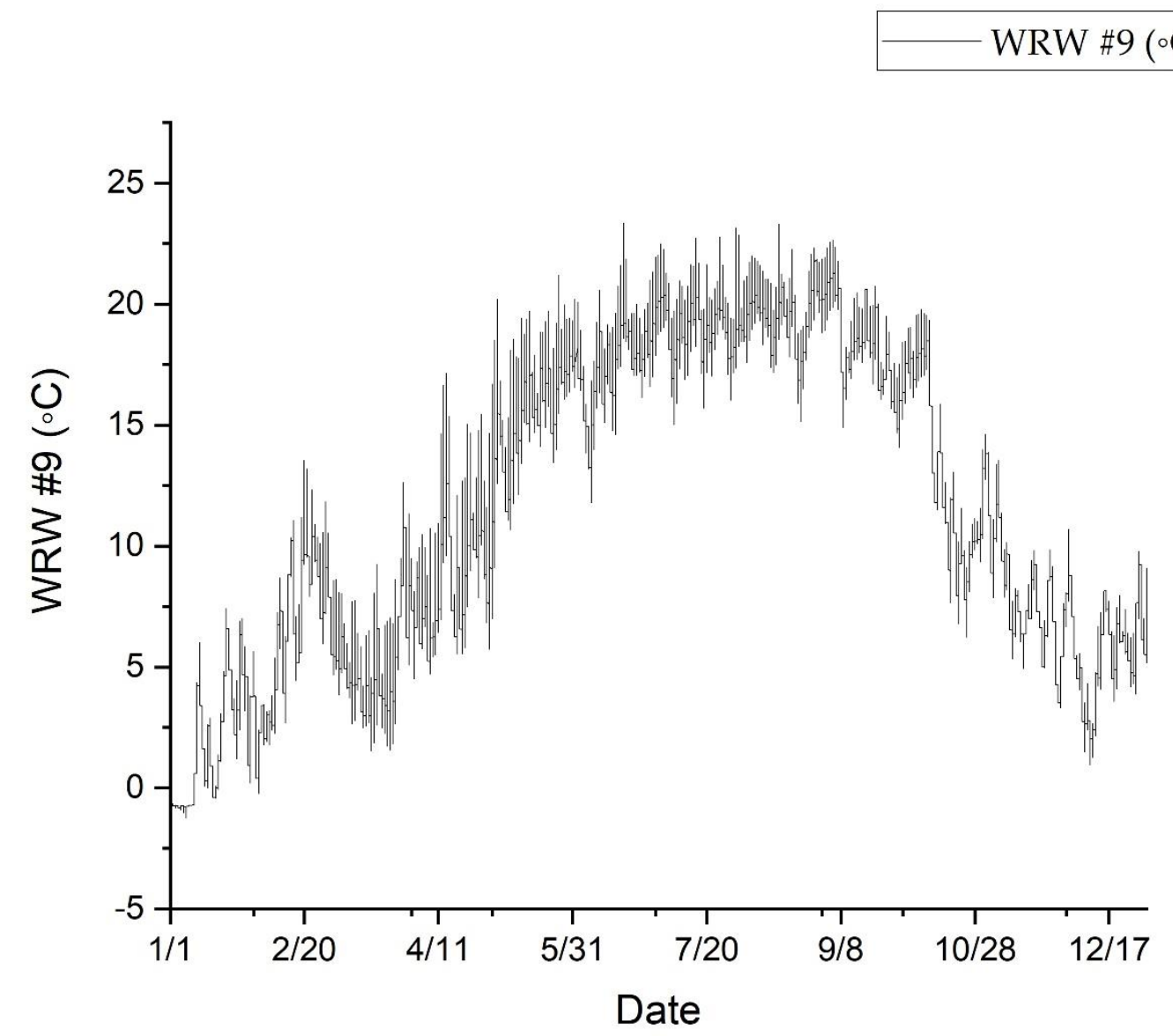

Figure B9. Time-series of steam water temperature across the 2018 annual year recorded at gauging site \#9 located in West Run Watershed, West Virginia, USA. 


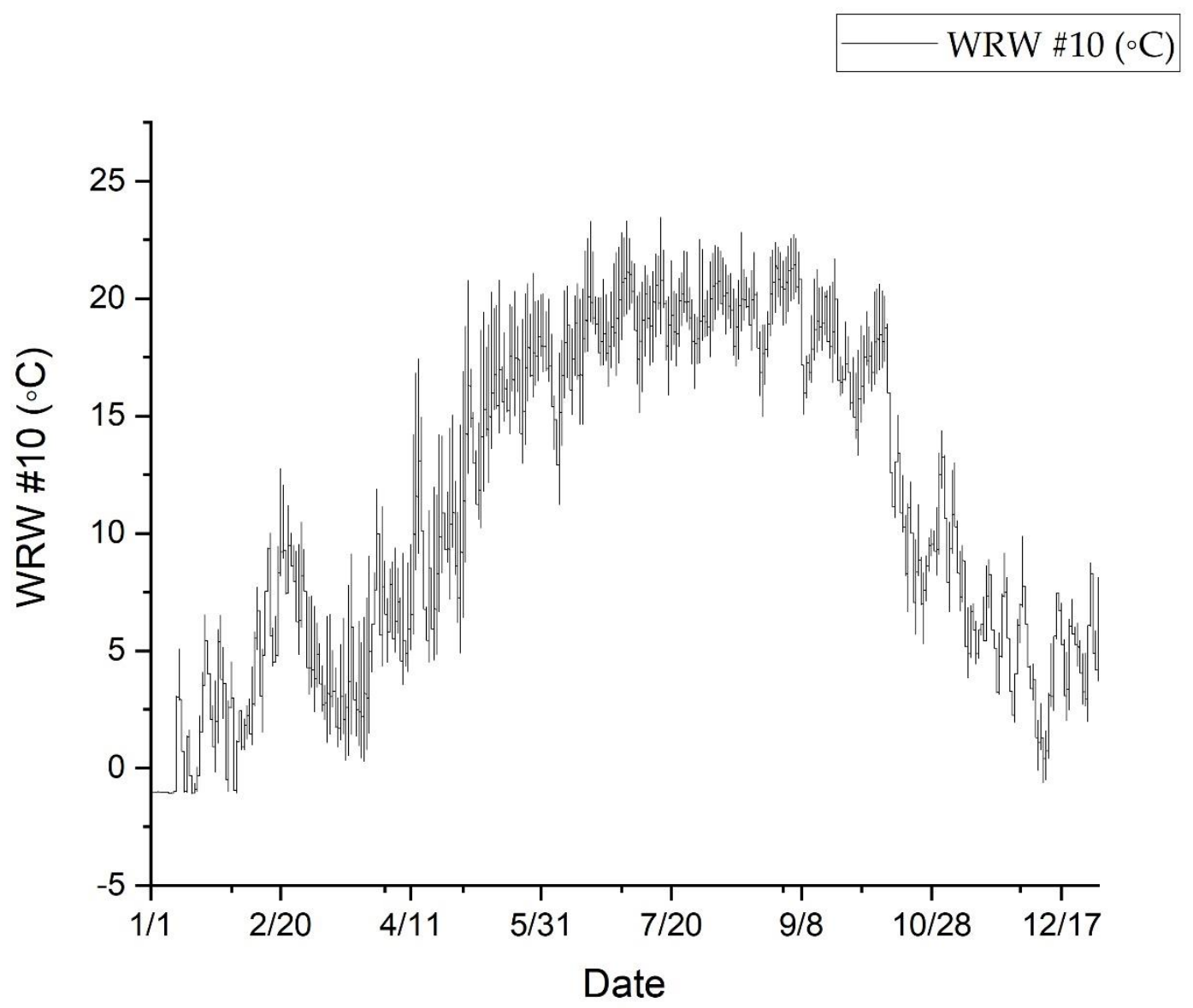

Figure B10. Time-series of steam water temperature across the 2018 annual year recorded at gauging site \#10 located in West Run Watershed, West Virginia, USA. 


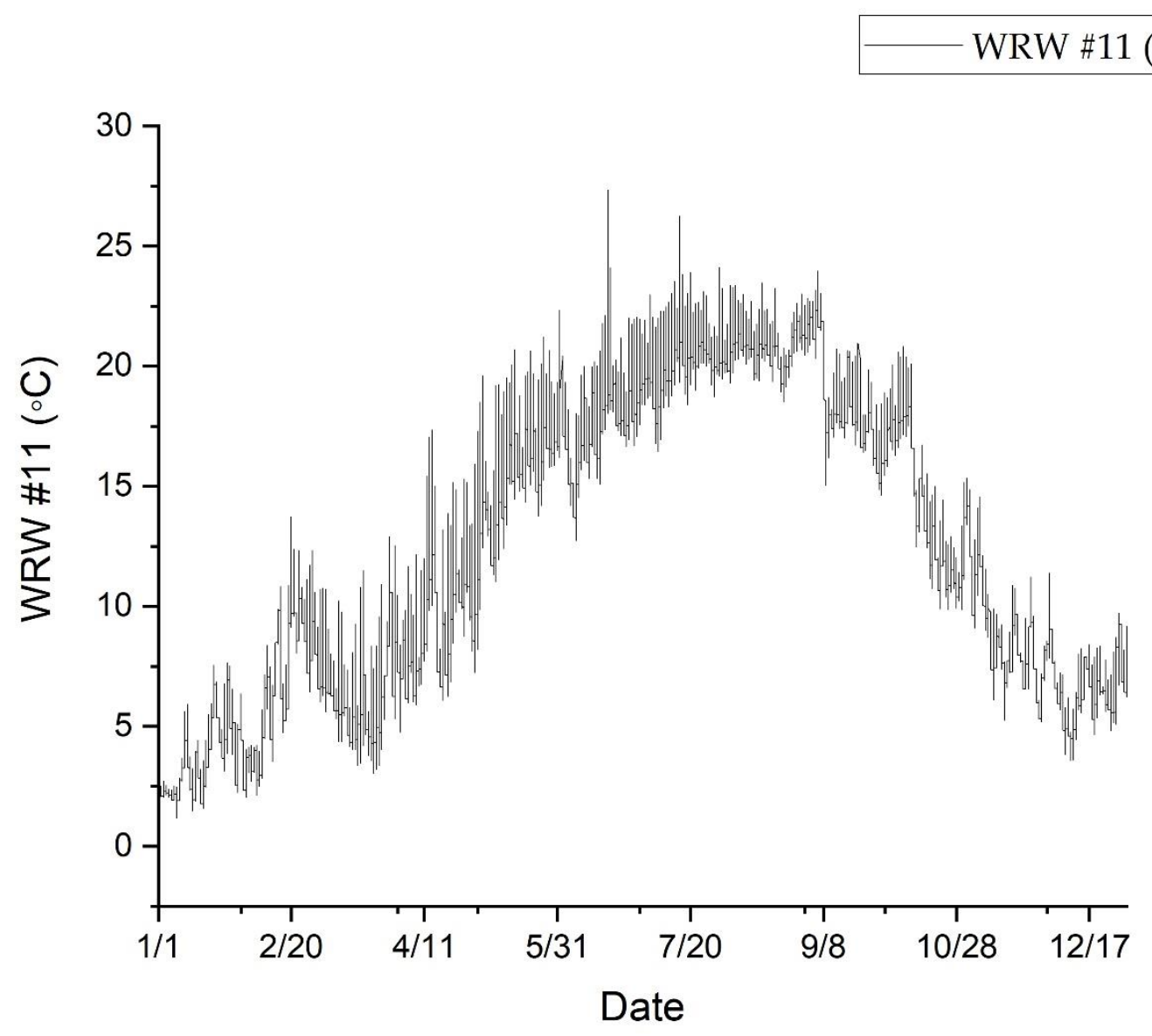

Figure B11. Time-series of steam water temperature across the 2018 annual year recorded at gauging site \#11 located in West Run Watershed, West Virginia, USA 


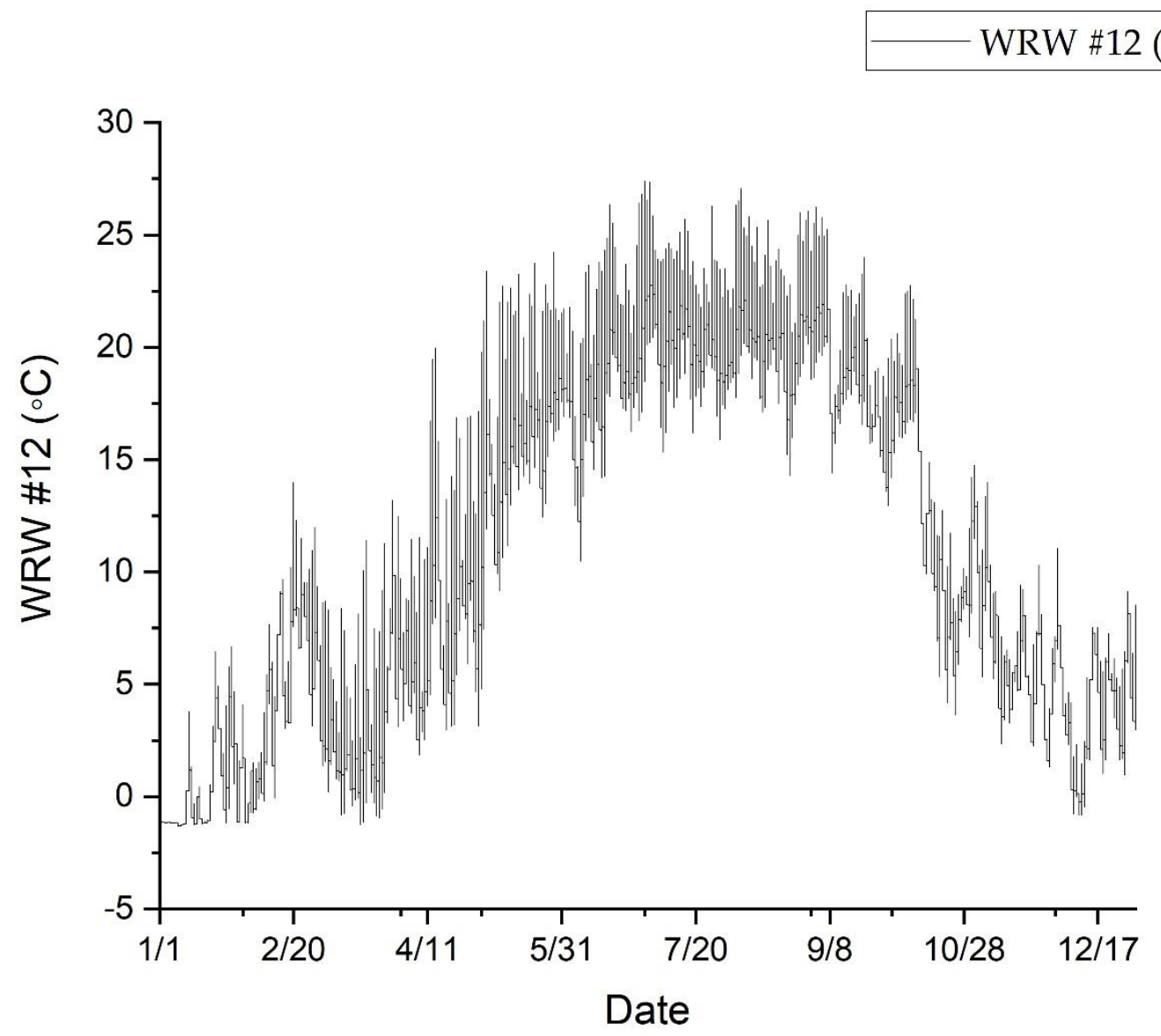

Figure B12. Time-series of steam water temperature across the 2018 annual year recorded at gauging site \#12 located in West Run Watershed, West Virginia, USA 


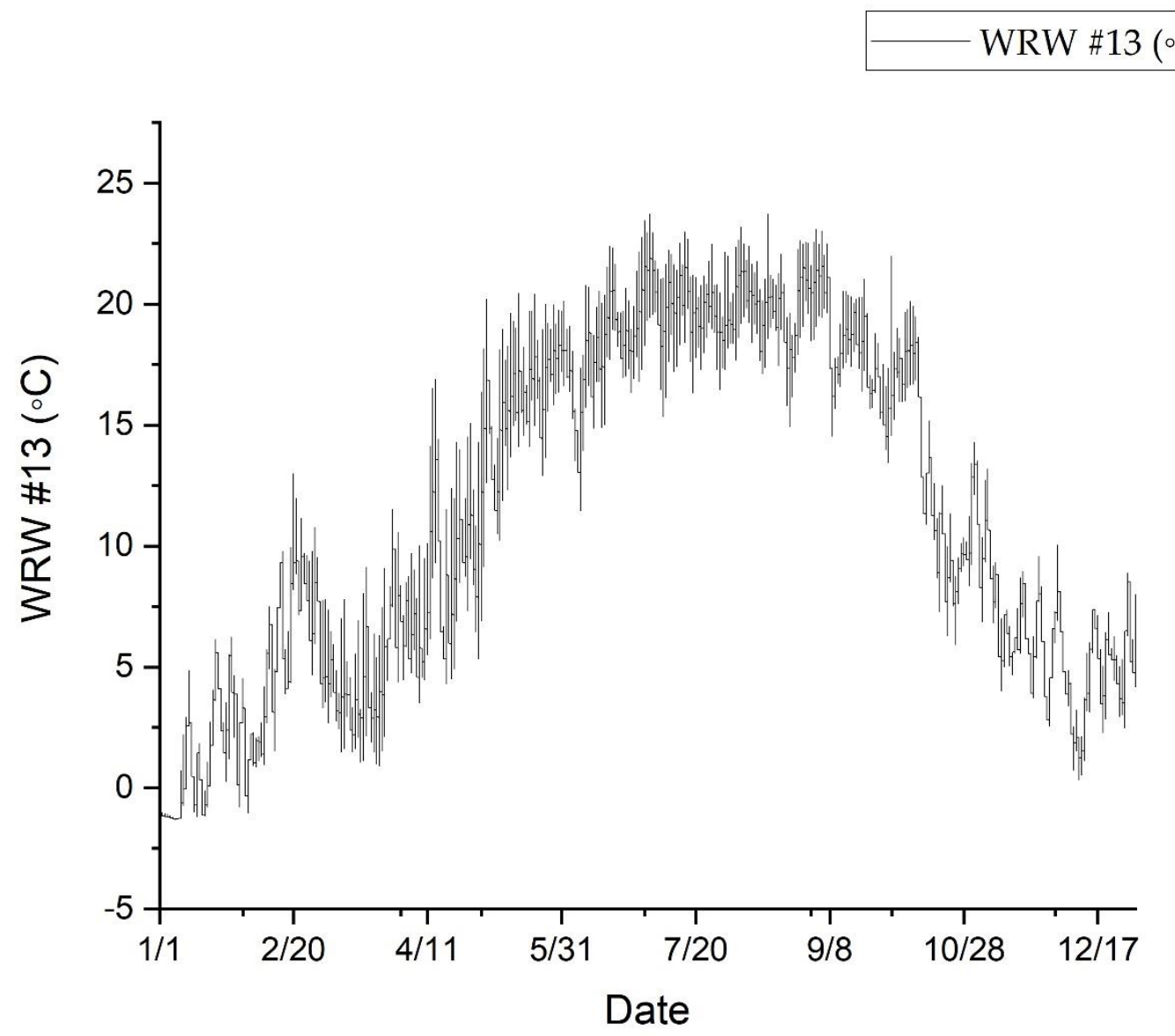

Figure B13. Time-series of steam water temperature across the 2018 annual year recorded at gauging site \#13 located in West Run Watershed, West Virginia, USA 


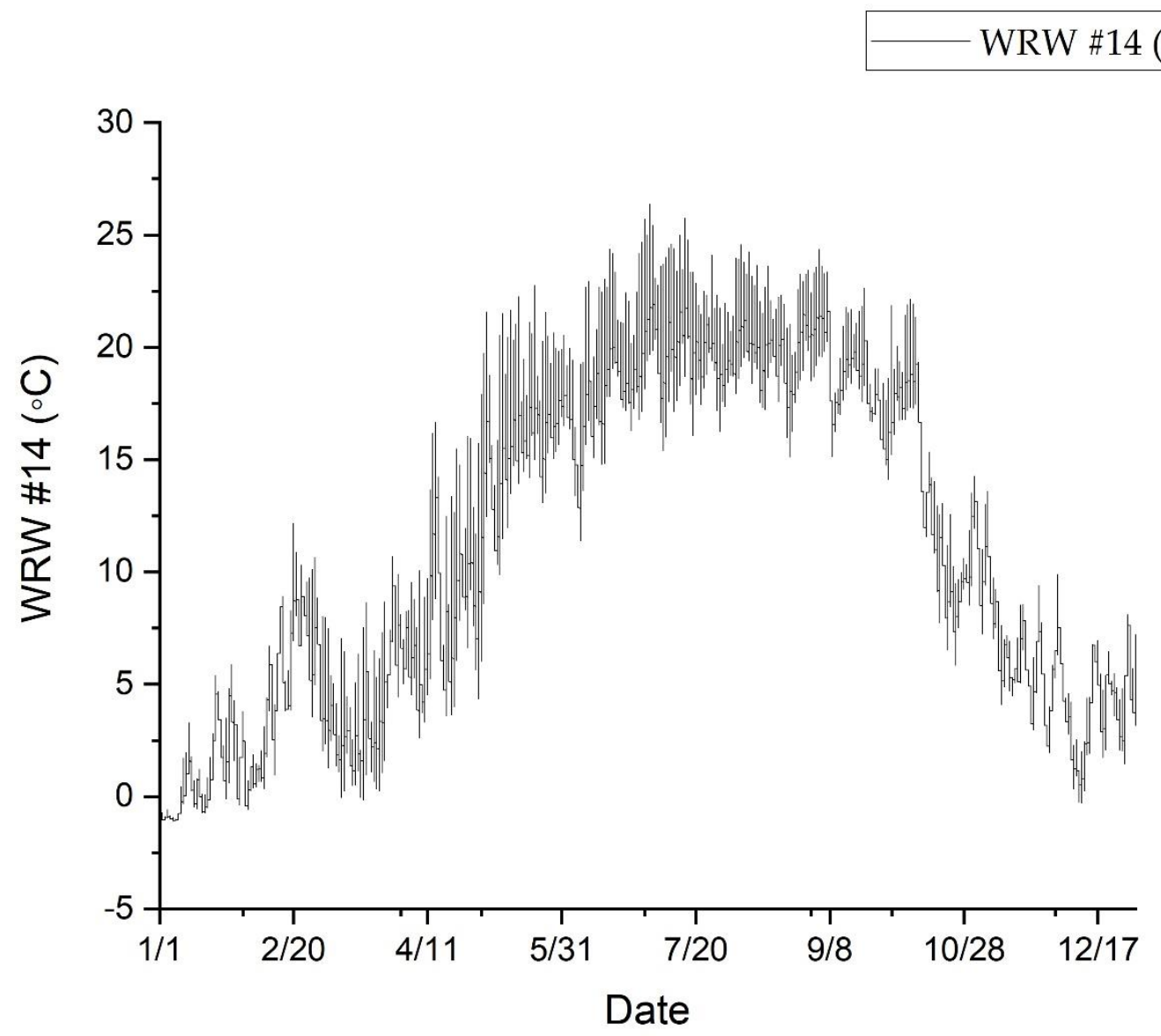

Figure B14. Time-series of steam water temperature across the 2018 annual year recorded at gauging site \#14 located in West Run Watershed, West Virginia, USA 


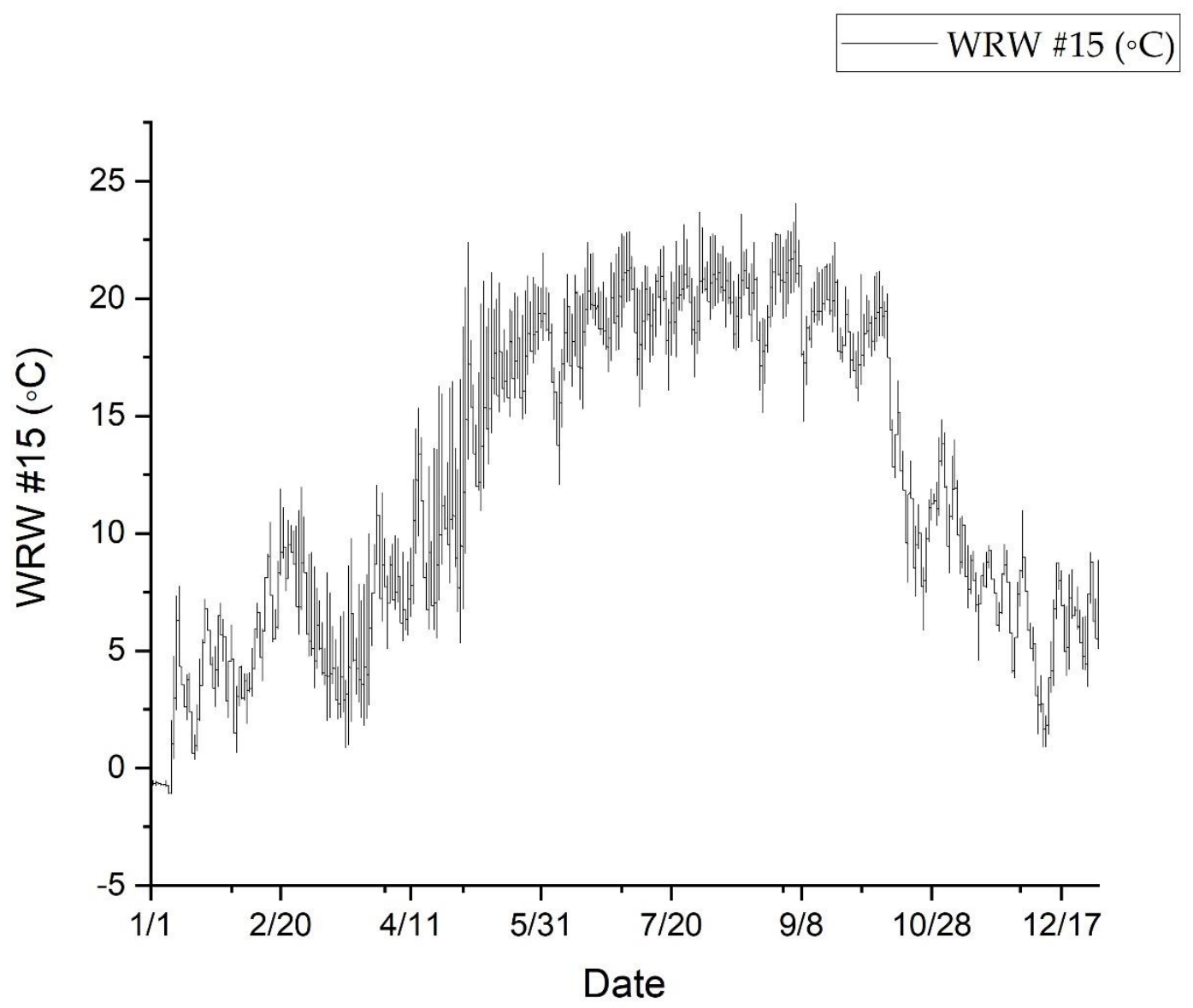

Figure B15. Time-series of steam water temperature across the 2018 annual year recorded at gauging site \#15 located in West Run Watershed, West Virginia, USA. 


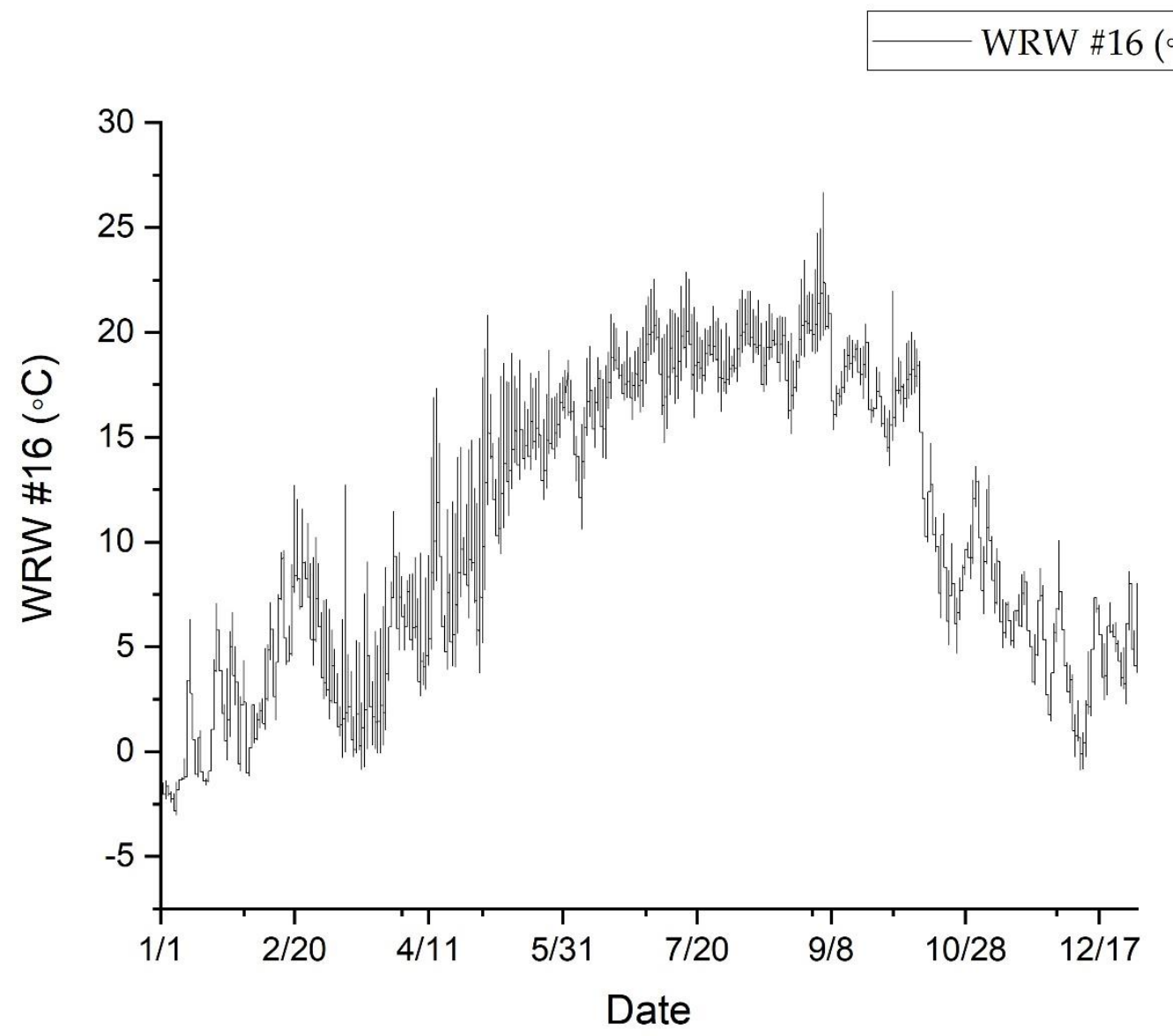

Figure B16. Time-series of steam water temperature across the 2018 annual year recorded at gauging site \#16 located in West Run Watershed, West Virginia, USA. 


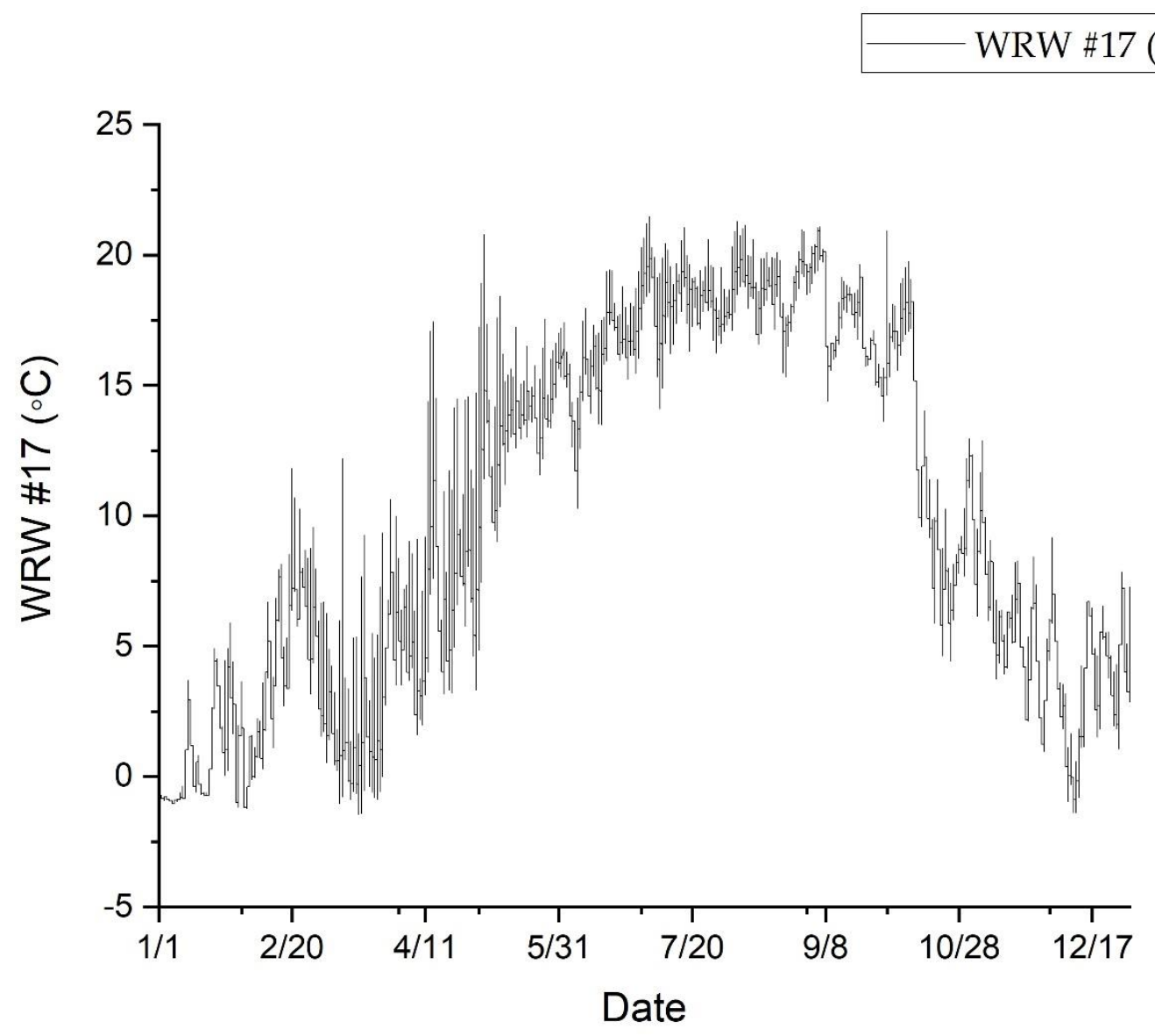

Figure B17. Time-series of steam water temperature across the 2018 annual year recorded at gauging site \#17 located in West Run Watershed, West Virginia, USA 


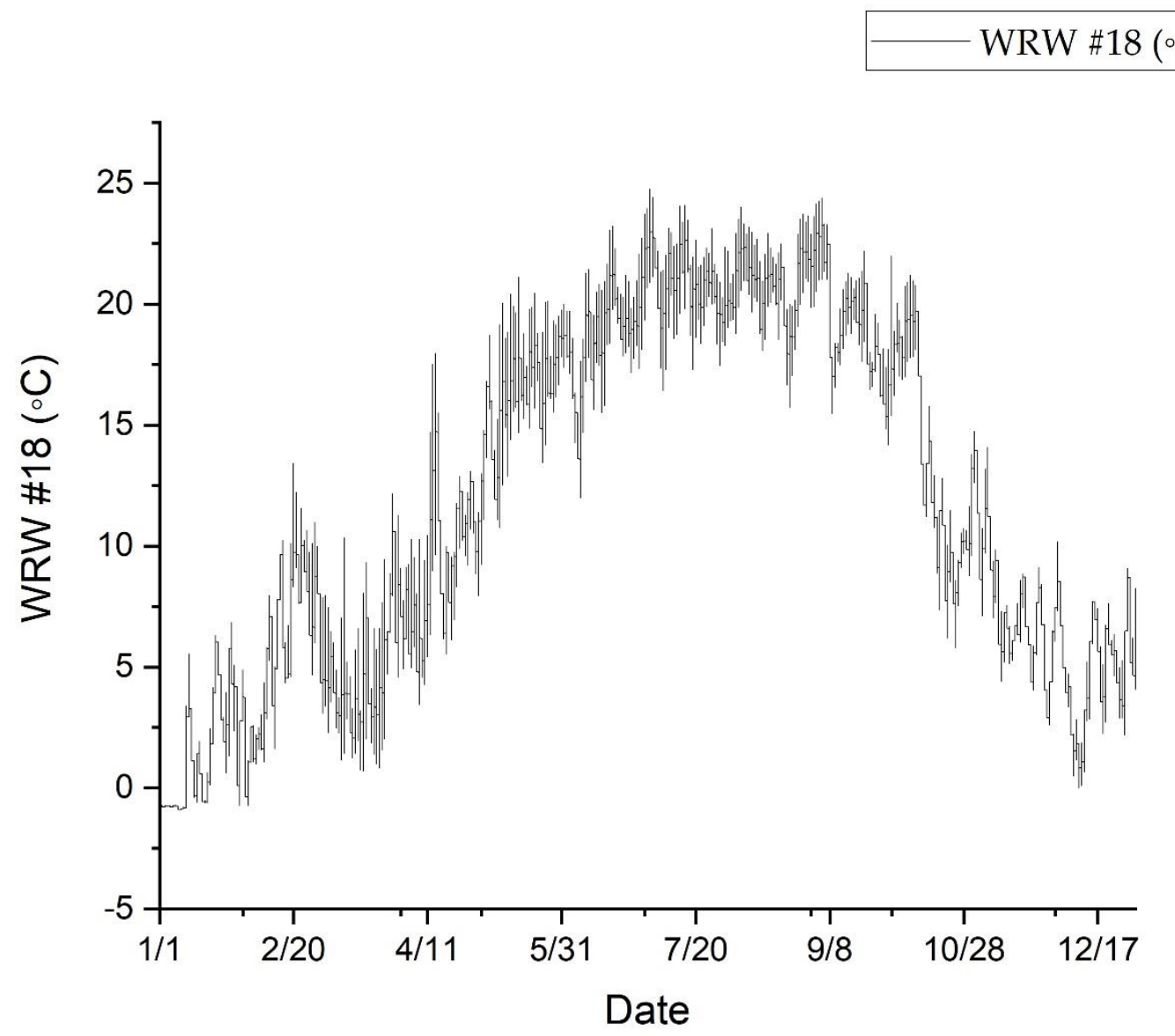

Figure B18. Time-series of steam water temperature across the 2018 annual year recorded at gauging site \#18 located in West Run Watershed, West Virginia, USA 


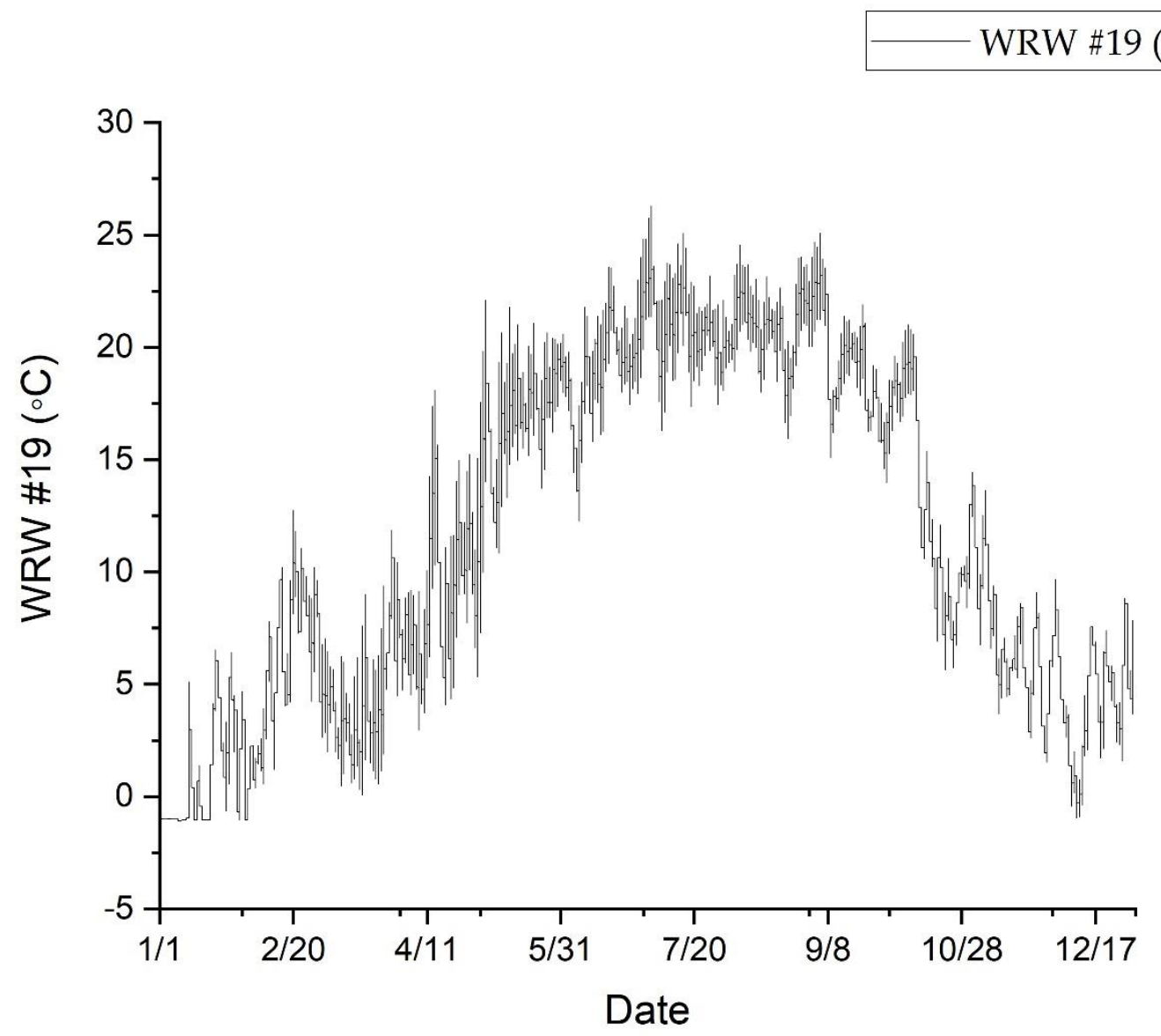

Figure B19. Time-series of steam water temperature across the 2018 annual year recorded at gauging site \#19 located in West Run Watershed, West Virginia, USA 


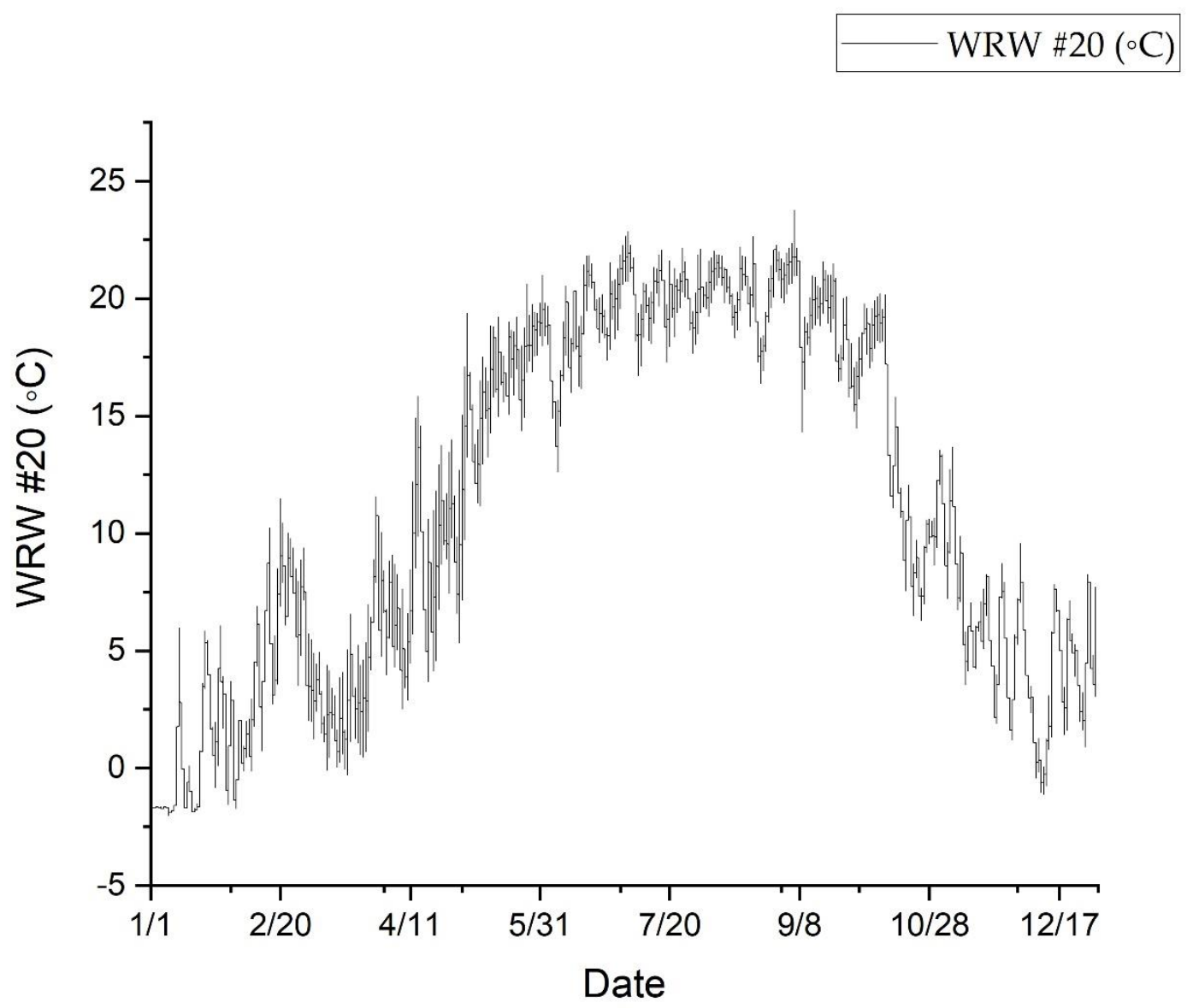

Figure B20. Time-series of steam water temperature across the 2018 annual year recorded at gauging site \#20 located in West Run Watershed, West Virginia, USA 


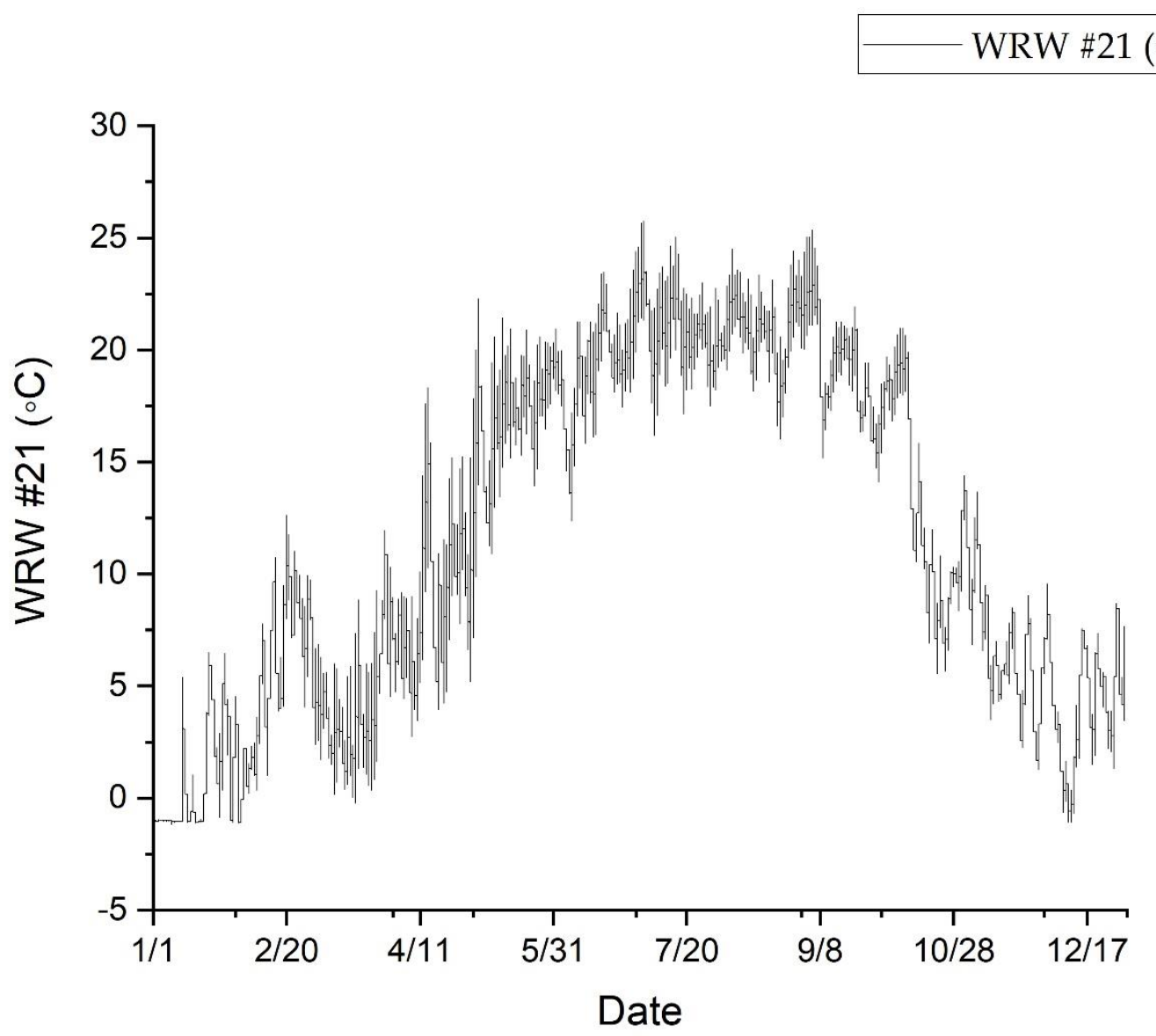

Figure B21. Time-series of steam water temperature across the 2018 annual year recorded at gauging site \#21 located in West Run Watershed, West Virginia, USA 


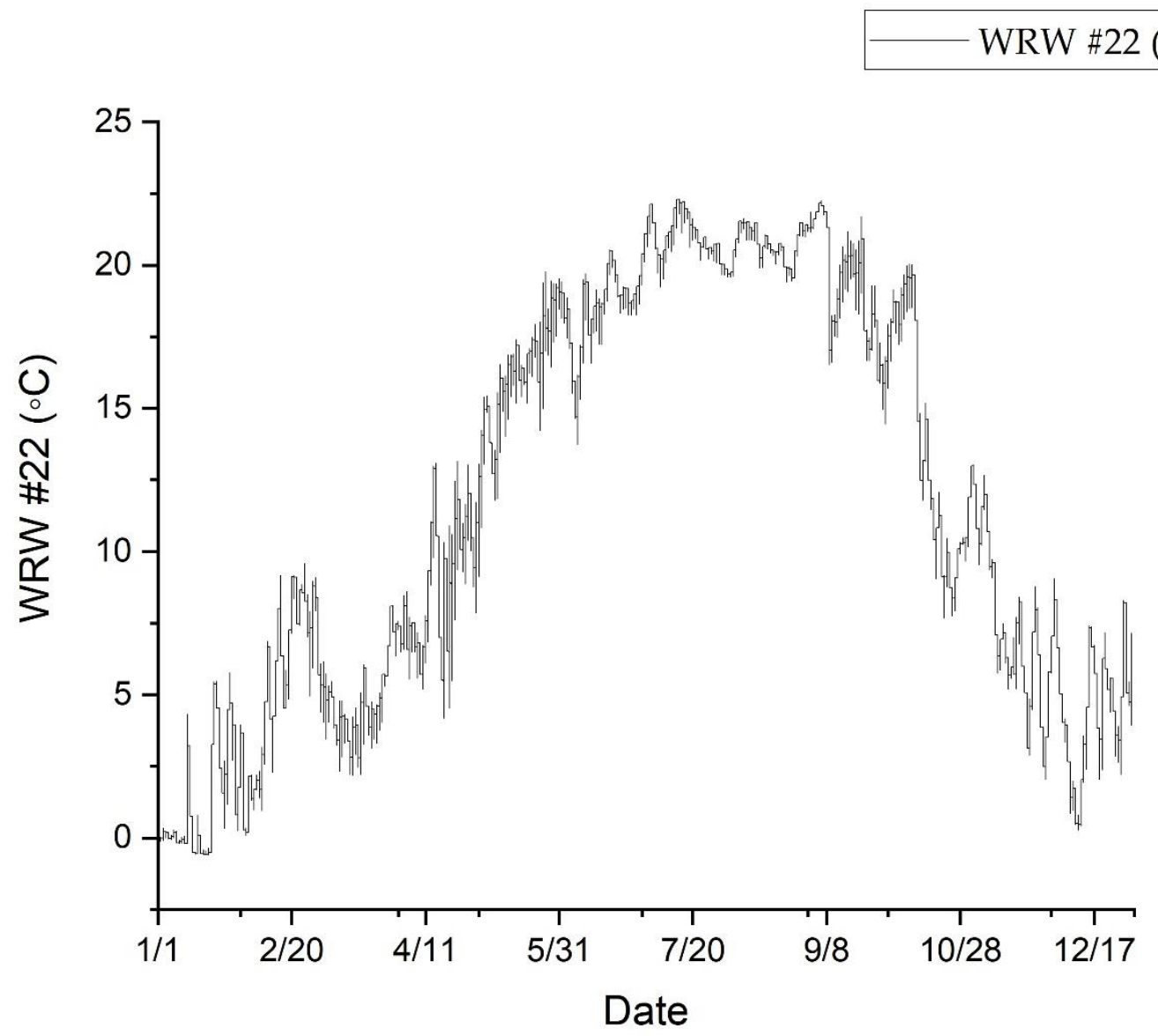

Figure B22. Time-series of steam water temperature across the 2018 annual year recorded at gauging site \#22 located in West Run Watershed, West Virginia, USA 


\section{APPENDIX C \\ SITE BY SITE ANALYSIS RESULTS}

\begin{tabular}{|c|c|c|c|}
\hline Site Comparison & $\begin{array}{l}\text { MEAN } \\
\text { p-value }\end{array}$ & $\begin{array}{c}\text { MAX } \\
\text { p-value }\end{array}$ & $\begin{array}{c}\text { MIN } \\
\text { p-value }\end{array}$ \\
\hline WRW \#1 ('C) "WRW \#5 (॰C)" & \multirow[b]{4}{*}{0.02} & 0.02 & \\
\hline WRW \#1 (C) "WRW \#11 (C)" & & 0.00 & \\
\hline WRW \#1 (C) "WRW \#12 (॰C)" & & 0.00 & \\
\hline WRW \#1 (C) "WRW \#15 (०C)" & & 0.01 & \\
\hline WRW \#2 (ㄷ) "WRW \#5 (ㄷ)" & 0.01 & 0.00 & \multirow{10}{*}{0.01} \\
\hline WRW \#2 (॰C) "WRW \#6 ( $\left.{ }^{\circ} \mathrm{C}\right) "$ & & 0.00 & \\
\hline WRW \#2 (ㄷ) "WRW \#9 ( $\left.{ }^{\circ} \mathrm{C}\right) "$ & & 0.01 & \\
\hline WRW \#2 (॰C) "WRW \#11 (C)" & & 0.00 & \\
\hline WRW \#2 (॰C) "WRW \#12 (॰C)" & & 0.00 & \\
\hline WRW \#2 $\left({ }^{\circ} \mathrm{C}\right)$ "WRW \#14 $\left({ }^{\circ} \mathrm{C}\right) "$ & & 0.00 & \\
\hline WRW \#2 (॰C) "WRW \#15 (C)" & 0.00 & 0.00 & \\
\hline WRW \#2 (C) "WRW \#18 (ㄷ)" & 0.01 & 0.00 & \\
\hline WRW \#2 (॰C) "WRW \#19 (C)" & 0.03 & 0.00 & \\
\hline WRW \#2 $\left({ }^{\circ} \mathrm{C}\right)$ "WRW \#21 $\left({ }^{\circ} \mathrm{C}\right) "$ & 0.04 & 0.01 & \\
\hline WRW \#3 ( $\left.{ }^{\circ} \mathrm{C}\right)$ "WRW \#5 (ㄷ)" & \multirow[b]{4}{*}{0.01} & 0.01 & \multirow[b]{8}{*}{0.00} \\
\hline WRW \#3 (॰C) "WRW \#11 (C)" & & 0.00 & \\
\hline WRW \#3 (॰C) "WRW \#12 (॰C)" & & 0.00 & \\
\hline WRW \#3 $\left({ }^{\circ} \mathrm{C}\right)$ "WRW \#15 $\left({ }^{\circ} \mathrm{C}\right) "$ & & 0.01 & \\
\hline WRW \#4 ('C) "WRW \#5 (ㄷ)" & \multirow[b]{4}{*}{0.00} & 0.01 & \\
\hline WRW \#4 (॰C) "WRW \#11 (॰C)" & & 0.00 & \\
\hline WRW \#4 (॰C) "WRW \#12 (C)" & & 0.00 & \\
\hline WRW \#4 $\left({ }^{\circ} \mathrm{C}\right)$ "WRW \#15 $\left({ }^{\circ} \mathrm{C}\right) "$ & & 0.01 & \\
\hline WRW \#5 ('C) "WRW \#17 ( $\left.{ }^{\circ} \mathrm{C}\right) "$ & 0.00 & 0.00 & 0.02 \\
\hline WRW \#6 ( $\left.{ }^{\circ} \mathrm{C}\right)$ "WRW \#17 (०C)" & 0.03 & 0.01 & \\
\hline WRW \#9 ( $\left.{ }^{\circ} \mathrm{C}\right)$ "WRW \#17 (C)" & 0.03 & & \\
\hline WRW \#11 (॰C) "WRW \#15 (०C)" & & \multirow[b]{2}{*}{0.00} & \multirow[t]{2}{*}{0.01} \\
\hline WRW \#11 ( $\left.{ }^{\circ} \mathrm{C}\right)$ "WRW \#17 $\left({ }^{\circ} \mathrm{C}\right) "$ & & & \\
\hline WRW \#12 (॰C) "WRW \#15 (०C)" & & \multirow[b]{2}{*}{0.00} & \multirow[t]{2}{*}{0.01} \\
\hline WRW \#12 (॰C) "WRW \#17 ( $\left.{ }^{\circ} \mathrm{C}\right) "$ & & & \\
\hline WRW \#14 (॰C) "WRW \#17 (०C)" & & 0.01 & \\
\hline WRW \#15 (॰C) "WRW \#16 (०C)" & 0.04 & & 0.04 \\
\hline WRW \#15 ( $\left.{ }^{\circ} \mathrm{C}\right)$ "WRW \#17 ( $\left({ }^{\circ} \mathrm{C}\right) "$ & 0.00 & 0.00 & 0.00 \\
\hline WRW \#17 ('C) "WRW \#18 (०C)" & 0.00 & 0.01 & \multirow[t]{3}{*}{0.02} \\
\hline WRW \#17 (॰C) "WRW \#19 (ㄷ)" & 0.01 & 0.02 & \\
\hline WRW \#17 (ㄷ) "WRW \#21 ( $\left.{ }^{\circ} \mathrm{C}\right) "$ & 0.02 & 0.02 & \\
\hline
\end{tabular}

Figure C1. Results of site by site annual comparison using a Kruskal Wallis ANOVA with a post hoc analysis of mean, maximum, and minimum daily Tw collected at twenty-one sites located in West Run Watershed, West Virginia, USA. Only significant $(\alpha=0.05) p$-value are shown. 


\begin{tabular}{|c|c|c|c|c|c|c|c|c|c|c|c|c|c|c|c|c|}
\hline & Q1 & Q2 & Q3 & Q4 & JAN & FEB & MAR & APR & MAY & JUN & JUL & AUG & SEP & OCT & NOV & DEC \\
\hline & p-value & $\mathrm{p}$-value & $\mathrm{p}$-value & p-value & p-value & $\mathrm{p}$-value & p-value & p-value & p-value & p-value & p-value & $\mathrm{p}$-value & p-value & p-value & p-value & $\mathrm{p}$-value \\
\hline WRW \#1 ( $\left.{ }^{\circ} \mathrm{C}\right) \quad$ "WRW \#2 ( $\left.{ }^{\circ} \mathrm{C}\right) "$ & & & & & & & 0.02 & & & & & & & & & 0.01 \\
\hline WRW \#1 ( $\left.{ }^{\circ} \mathrm{C}\right) \quad$ "WRW \#3 $\left({ }^{\circ} \mathrm{C}\right) "$ & 0.01 & & 0.01 & & & & 0.01 & & & & & & & & & 0.00 \\
\hline WRW \#1 ( $\left.{ }^{\circ} \mathrm{C}\right)$ "WRW \#4 (॰C)" & 0.00 & & 0.00 & 0.00 & 0.00 & & 0.00 & & & & 0.04 & & & & & 0.00 \\
\hline WRW \#1 (॰C) "WRW \#5 (॰C)" & 0.00 & 0.00 & 0.00 & 0.01 & 0.00 & & 0.00 & & 0.00 & 0.00 & 0.00 & 0.00 & 0.00 & & 0.01 & 0.00 \\
\hline WRW \#1 $\left({ }^{\circ} \mathrm{C}\right) \quad$ "WRW \#6 $\left({ }^{\circ} \mathrm{C}\right) "$ & 0.00 & 0.00 & 0.00 & & 0.01 & & 0.00 & & 0.00 & 0.00 & 0.00 & 0.00 & 0.00 & & & 0.00 \\
\hline WRW \#1 (॰C) "WRW \#7 (॰C)" & 0.00 & & 0.00 & & 0.00 & & 0.01 & & & & 0.00 & 0.00 & 0.00 & & & 0.00 \\
\hline WRW \#1 (॰C) "WRW \#8 (॰C)" & & & 0.00 & & & & & & & & & & 0.03 & & & \\
\hline WRW \#1 (॰C) "WRW \#9 (॰C)" & & & 0.00 & & & & & & 0.01 & 0.00 & 0.00 & 0.00 & 0.00 & & & \\
\hline WRW \#1 ( $\left.{ }^{\circ} \mathrm{C}\right)$ "WRW \#10 $\left({ }^{\circ} \mathrm{C}\right) "$ & 0.00 & & 0.00 & 0.05 & 0.00 & & 0.00 & & 0.00 & 0.00 & 0.00 & 0.00 & 0.00 & & & 0.00 \\
\hline WRW \#1 ( $\left.{ }^{\circ} \mathrm{C}\right)$ "WRW \#11 $\left({ }^{\circ} \mathrm{C}\right) "$ & 0.00 & 0.01 & 0.00 & 0.00 & 0.00 & & 0.00 & & 0.00 & 0.00 & 0.00 & 0.00 & 0.00 & & 0.01 & 0.00 \\
\hline WRW \#1 ( $\left.{ }^{\circ} \mathrm{C}\right)$ "WRW \#12 (॰C)" & 0.00 & 0.01 & 0.00 & 0.00 & 0.00 & & 0.00 & & 0.00 & 0.00 & 0.00 & 0.00 & 0.00 & & 0.01 & 0.00 \\
\hline WRW \#1 ( $\left.{ }^{\circ} \mathrm{C}\right)$ "WRW \#13 (॰C)" & 0.00 & & 0.00 & & 0.00 & & 0.05 & & 0.00 & 0.00 & 0.00 & 0.00 & 0.00 & & & 0.00 \\
\hline WRW \#1 ( $\left.{ }^{\circ} \mathrm{C}\right)$ "WRW \#14 $\left({ }^{\circ} \mathrm{C}\right) "$ & 0.00 & 0.02 & 0.00 & 0.03 & 0.00 & & 0.00 & & 0.00 & 0.00 & 0.00 & 0.00 & 0.00 & & & 0.00 \\
\hline WRW \#1 ( $\left.{ }^{\circ} \mathrm{C}\right)$ "WRW \#15 $\left({ }^{\circ} \mathrm{C}\right) "$ & & 0.00 & 0.00 & & & & & & 0.00 & 0.00 & 0.00 & 0.00 & 0.00 & & & \\
\hline WRW \#1 ( $\left.{ }^{\circ} \mathrm{C}\right)$ "WRW \#16 $\left({ }^{\circ} \mathrm{C}\right) "$ & 0.00 & & 0.00 & 0.01 & 0.00 & & 0.00 & & & & 0.00 & 0.00 & 0.00 & & & 0.00 \\
\hline WRW \#1 ( $\left.{ }^{\circ} \mathrm{C}\right)$ "WRW \#17 $\left({ }^{\circ} \mathrm{C}\right) "$ & 0.00 & & 0.00 & 0.00 & 0.00 & & 0.00 & & & & & & & & 0.00 & 0.00 \\
\hline WRW \#1 ( $\left.{ }^{\circ} \mathrm{C}\right)$ "WRW \#18 $\left({ }^{\circ} \mathrm{C}\right) "$ & 0.00 & 0.00 & 0.00 & & 0.00 & & & & 0.00 & 0.00 & 0.00 & 0.00 & 0.00 & & & 0.00 \\
\hline WRW \#1 ( $\left.{ }^{\circ} \mathrm{C}\right)$ "WRW \#19 $\left({ }^{\circ} \mathrm{C}\right) "$ & 0.00 & 0.00 & 0.00 & 0.04 & 0.00 & & 0.00 & & 0.00 & 0.00 & 0.00 & 0.00 & 0.00 & & & 0.00 \\
\hline WRW \#1 ( $\left.{ }^{\circ} \mathrm{C}\right)$ "WRW \#20 $\left({ }^{\circ} \mathrm{C}\right) "$ & 0.00 & 0.01 & 0.00 & 0.01 & 0.00 & & 0.00 & & 0.00 & 0.00 & 0.00 & 0.00 & 0.00 & & 0.04 & 0.00 \\
\hline WRW \#1 ( $\left.{ }^{\circ} \mathrm{C}\right)$ "WRW \#21 $\left({ }^{\circ} \mathrm{C}\right) "$ & 0.00 & 0.00 & 0.00 & 0.02 & 0.00 & & 0.00 & & 0.00 & 0.00 & 0.00 & 0.00 & 0.00 & & & 0.00 \\
\hline WRW \#2 ('C) "WRW \#4 (॰C)" & & & 0.00 & & 0.00 & & & & & & & & & & & \\
\hline WRW \#2 (॰C) "WRW \#5 (॰C)" & & 0.00 & 0.00 & & & & & & 0.00 & 0.00 & 0.00 & 0.00 & 0.00 & & & \\
\hline WRW \#2 (॰C) "WRW \#6 (॰C)" & & 0.01 & 0.00 & & & & & & 0.00 & 0.00 & 0.00 & 0.00 & 0.00 & & & \\
\hline WRW \#2 (॰C) "WRW \#7 (॰C)" & & & 0.00 & & & & & & & & 0.01 & 0.00 & 0.01 & & & \\
\hline WRW \#2 (॰C) "WRW \#8 ( $\left.{ }^{\circ} \mathrm{C}\right) "$ & & & 0.00 & & & & & & & & & & & & & \\
\hline WRW \#2 ( $\left.{ }^{\circ} \mathrm{C}\right)$ "WRW \#9 (C)" & & & 0.00 & & & & & & 0.02 & 0.04 & 0.00 & 0.00 & 0.00 & & & \\
\hline WRW \#2 ( $\left.{ }^{\circ} \mathrm{C}\right)$ "WRW \#10 $\left({ }^{\circ} \mathrm{C}\right) "$ & & & 0.00 & & & & & & 0.00 & 0.00 & 0.00 & 0.00 & 0.00 & & & \\
\hline WRW \#2 ( $\left.{ }^{\circ} \mathrm{C}\right)$ "WRW \#11 $\left({ }^{\circ} \mathrm{C}\right) "$ & & 0.02 & 0.00 & & 0.00 & & & & 0.00 & 0.00 & 0.00 & 0.00 & 0.00 & & & \\
\hline WRW \#2 ( $\left.{ }^{\circ} \mathrm{C}\right)$ "WRW \#12 $\left({ }^{\circ} \mathrm{C}\right) "$ & & 0.02 & 0.00 & & 0.00 & & & & 0.00 & 0.00 & 0.00 & 0.00 & 0.00 & & & \\
\hline WRW \#2 ( $\left.{ }^{\circ} \mathrm{C}\right)$ "WRW \#13 $\left({ }^{\circ} \mathrm{C}\right) "$ & & & 0.00 & & & & & & 0.01 & 0.00 & 0.00 & 0.00 & 0.00 & & & \\
\hline WRW \#2 ( $\left.{ }^{\circ} \mathrm{C}\right)$ "WRW \#14 $\left({ }^{\circ} \mathrm{C}\right) "$ & & & 0.00 & & & & & & 0.00 & 0.00 & 0.00 & 0.00 & 0.00 & & & \\
\hline WRW \#2 ( $\left.{ }^{\circ} \mathrm{C}\right)$ "WRW \#15 $\left({ }^{\circ} \mathrm{C}\right) "$ & & 0.00 & 0.00 & & & & & & 0.00 & 0.00 & 0.00 & 0.00 & 0.00 & & & \\
\hline WRW \#2 ( $\left.{ }^{\circ} \mathrm{C}\right)$ "WRW \#16 $\left({ }^{\circ} \mathrm{C}\right) "$ & & & 0.00 & & 0.01 & & & & & & 0.00 & 0.00 & 0.01 & & & \\
\hline WRW \#2 ( $\left.{ }^{\circ} \mathrm{C}\right)$ "WRW \#17 $\left({ }^{\circ} \mathrm{C}\right) "$ & 0.01 & & 0.00 & & & & 0.02 & & & & & & & & & \\
\hline WRW \#2 ( $\left.{ }^{\circ} \mathrm{C}\right)$ "WRW \#18 $\left({ }^{\circ} \mathrm{C}\right) "$ & & 0.01 & 0.00 & & & & & & 0.00 & 0.00 & 0.00 & 0.00 & 0.00 & & & \\
\hline WRW \#2 (॰C) "WRW \#19 (॰C)" & & 0.00 & 0.00 & & 0.03 & & & & 0.00 & 0.00 & 0.00 & 0.00 & 0.00 & & & \\
\hline WRW \#2 ( $\left.{ }^{\circ} \mathrm{C}\right)$ "WRW \#20 $\left({ }^{\circ} \mathrm{C}\right) "$ & & 0.04 & 0.00 & & 0.00 & & & & 0.00 & 0.00 & 0.00 & 0.00 & 0.00 & & & \\
\hline WRW \#2 ( $\left.{ }^{\circ} \mathrm{C}\right)$ "WRW \#21 $\left({ }^{\circ} \mathrm{C}\right) "$ & & 0.00 & 0.00 & & 0.01 & & & & 0.00 & 0.00 & 0.00 & 0.00 & 0.00 & & & \\
\hline WRW \#3 (C) "WRW \#5 (ㄷ)" & & 0.01 & 0.00 & & & & & & 0.00 & 0.00 & 0.00 & 0.00 & 0.01 & & & \\
\hline WRW \#3 (॰C) "WRW \#6 (॰C)" & & & 0.00 & & & & & & & & 0.00 & 0.00 & & & & \\
\hline WRW \#3 (॰C) "WRW \#7 (॰C)" & & & 0.00 & & & & & & & & & & & & & \\
\hline WRW \#3 ( $\left.{ }^{\circ} \mathrm{C}\right)$ "WRW \#9 (C)" & & & 0.00 & & & & & & & & 0.02 & 0.02 & & & & \\
\hline WRW \#3 ( $\left.{ }^{\circ} \mathrm{C}\right)$ "WRW \#10 $\left({ }^{\circ} \mathrm{C}\right) "$ & & & 0.00 & & & & & & & & 0.00 & 0.01 & & & & \\
\hline WRW \#3 ( $\left.{ }^{\circ} \mathrm{C}\right)$ "WRW \#11 $\left({ }^{\circ} \mathrm{C}\right) "$ & & & 0.00 & & & & & & & 0.00 & 0.00 & 0.00 & & & & \\
\hline WRW \#3 ( $\left.{ }^{\circ} \mathrm{C}\right)$ "WRW \#12 $\left({ }^{\circ} \mathrm{C}\right) "$ & & & 0.00 & & & & & & & 0.00 & 0.00 & 0.00 & & & & \\
\hline WRW \#3 (॰C) "WRW \#13 (॰C)" & & & 0.00 & & & & & & & & 0.00 & 0.00 & & & & \\
\hline
\end{tabular}

Figure C2. Results of site by site quarterly and monthly comparison using a Kruskal Wallis

ANOVA with a post hoc analysis of mean daily Tw collected at twenty-one sites located in West

Run Watershed, West Virginia, USA. Only significant $(\alpha=0.05)$ p-value are shown. (Part 1) 


\begin{tabular}{|c|c|c|c|c|c|c|c|c|c|c|c|c|c|c|}
\hline & Q1 & Q2 & Q3 & Q4 & JAN & FEB & MAR & APR & MAY & JUN & JUL & AUG & SEP & OCT NOV DEC \\
\hline & $\mathrm{p}$-value & $\mathrm{p}$-value & $\mathrm{p}$-value & $\mathrm{p}$-value & p-value & $\mathrm{p}$-value & $\mathrm{p}$-value & $\mathrm{p}$-value & $\mathrm{p}$-value & $\mathrm{p}$-value & $\mathrm{p}$-value & $\mathrm{p}$-value & $\mathrm{p}$-value & $p$-value $p$-value $p$-value \\
\hline WRW \#3 $\left({ }^{\circ} \mathrm{C}\right)$ "WRW \#14 ( $\left.{ }^{\circ} \mathrm{C}\right) "$ & & & 0.00 & & & & & & & & 0.00 & 0.00 & & \\
\hline WRW \#3 $\left({ }^{\circ} \mathrm{C}\right)$ "WRW \#15 $\left({ }^{\circ} \mathrm{C}\right) "$ & & & 0.00 & & & & & & 0.02 & 0.01 & 0.00 & 0.00 & 0.00 & \\
\hline WRW \#3 $\left({ }^{\circ} \mathrm{C}\right)$ "WRW \#16 $\left({ }^{\circ} \mathrm{C}\right) "$ & & & 0.00 & & & & & & & & & & & \\
\hline WRW \#3 $\left({ }^{\circ} \mathrm{C}\right)$ "WRW \#17 $\left({ }^{\circ} \mathrm{C}\right) "$ & & & & & & & 0.02 & & & & & & & \\
\hline WRW \#3 $\left({ }^{\circ} \mathrm{C}\right)$ "WRW \#18 $\left({ }^{\circ} \mathrm{C}\right) "$ & & & 0.00 & & & & & & & 0.01 & 0.00 & 0.00 & 0.01 & \\
\hline WRW \#3 $\left({ }^{\circ} \mathrm{C}\right)$ "WRW \#19 $\left({ }^{\circ} \mathrm{C}\right) "$ & & & 0.00 & & & & & & 0.01 & 0.00 & 0.00 & 0.00 & & \\
\hline WRW \#3 $\left({ }^{\circ} \mathrm{C}\right)$ "WRW \#20 $\left({ }^{\circ} \mathrm{C}\right) "$ & & & 0.00 & & 0.04 & & & & & 0.02 & 0.00 & 0.00 & 0.01 & \\
\hline WRW \#3 $\left({ }^{\circ} \mathrm{C}\right)$ "WRW \#21 $\left({ }^{\circ} \mathrm{C}\right) "$ & & & 0.00 & & & & & & 0.00 & 0.00 & 0.00 & 0.00 & 0.02 & \\
\hline WRW \#4 (॰C) "WRW \#5 (०C)" & & 0.00 & 0.00 & & & & & & 0.00 & 0.00 & 0.00 & 0.00 & & \\
\hline WRW \#4 $\left({ }^{\circ} \mathrm{C}\right)$ "WRW \#6 $\left({ }^{\circ} \mathrm{C}\right) "$ & & & 0.00 & & & & & & 0.04 & & 0.00 & 0.01 & & \\
\hline WRW \#4 $\left({ }^{\circ} \mathrm{C}\right)$ "WRW \#8 $\left({ }^{\circ} \mathrm{C}\right) "$ & 0.01 & & & & & & 0.00 & & & & & & & \\
\hline WRW \#4 $\left({ }^{\circ} \mathrm{C}\right)$ "WRW \#9 $\left({ }^{\circ} \mathrm{C}\right) "$ & & & 0.02 & & & & 0.00 & & & & & & & \\
\hline WRW \#4 $\left({ }^{\circ} \mathrm{C}\right)$ "WRW \#10 $\left({ }^{\circ} \mathrm{C}\right) "$ & & & 0.00 & & & & & & & & & & & \\
\hline WRW \#4 $\left({ }^{\circ} \mathrm{C}\right)$ "WRW \#11 ( $\left.{ }^{\circ} \mathrm{C}\right) "$ & & & 0.00 & & & & & & & 0.00 & 0.00 & 0.00 & & \\
\hline WRW \#4 ( $\left.{ }^{\circ} \mathrm{C}\right)$ "WRW \#12 $\left({ }^{\circ} \mathrm{C}\right) "$ & & & 0.00 & & & & & & & 0.00 & 0.00 & 0.00 & & \\
\hline WRW \#4 ( $\left.{ }^{\circ} \mathrm{C}\right)$ "WRW \#13 ( $\left.{ }^{\circ} \mathrm{C}\right) "$ & & & 0.00 & & & & & & & & & & & \\
\hline WRW \#4 ( $\left.{ }^{\circ} \mathrm{C}\right)$ "WRW \#14( $\left({ }^{\circ} \mathrm{C}\right) "$ & & & 0.00 & & & & & & & & 0.00 & 0.00 & & \\
\hline WRW \#4 $\left({ }^{\circ} \mathrm{C}\right)$ "WRW \#15( $\left({ }^{\circ} \mathrm{C}\right) "$ & 0.00 & 0.05 & 0.00 & & 0.01 & & 0.00 & & 0.00 & 0.00 & 0.04 & 0.00 & 0.01 & \\
\hline WRW \#4 $\left({ }^{\circ} \mathrm{C}\right)$ "WRW \#18 $\left({ }^{\circ} \mathrm{C}\right) "$ & & & 0.00 & & & & & & 0.04 & 0.00 & 0.00 & 0.00 & & \\
\hline WRW \#4 ( $\left.{ }^{\circ} \mathrm{C}\right)$ "WRW \#19 $\left({ }^{\circ} \mathrm{C}\right) "$ & & 0.05 & 0.00 & & & & & & 0.00 & 0.00 & 0.00 & 0.00 & & \\
\hline WRW \#4 $\left({ }^{\circ} \mathrm{C}\right)$ "WRW \#20 ( $\left.{ }^{\circ} \mathrm{C}\right) "$ & & & 0.00 & & & & & & & 0.02 & 0.01 & 0.00 & & \\
\hline WRW \#4 $\left({ }^{\circ} \mathrm{C}\right)$ "WRW \#21 $\left({ }^{\circ} \mathrm{C}\right) "$ & & 0.03 & 0.00 & & & & & & 0.00 & 0.00 & 0.00 & 0.00 & & \\
\hline 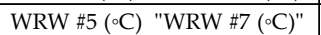 & & 0.02 & 0.00 & & & & & & 0.00 & 0.00 & 0.00 & & & \\
\hline WRW \#5 $\left({ }^{\circ} \mathrm{C}\right)$ "WRW \#8 $\left({ }^{\circ} \mathrm{C}\right) "$ & & & 0.00 & & & & & & 0.01 & 0.00 & 0.00 & 0.00 & & \\
\hline WRW \#5 $\left({ }^{\circ} \mathrm{C}\right)$ "WRW \#9 $\left({ }^{\circ} \mathrm{C}\right) "$ & & & 0.00 & & & & & & & 0.01 & 0.01 & & & \\
\hline WRW \#5 $\left({ }^{\circ} \mathrm{C}\right)$ "WRW \#10 $\left({ }^{\circ} \mathrm{C}\right) "$ & & & 0.03 & & & & & & & & & & & \\
\hline WRW \#5 $\left({ }^{\circ} \mathrm{C}\right)$ "WRW \#16 $\left({ }^{\circ} \mathrm{C}\right) "$ & & 0.00 & 0.00 & & & & & & 0.00 & 0.00 & 0.00 & & & \\
\hline WRW \#5 ( $\left.{ }^{\circ} \mathrm{C}\right)$ "WRW \#17 $\left({ }^{\circ} \mathrm{C}\right) "$ & & 0.00 & 0.00 & & & & & & 0.00 & 0.00 & 0.00 & 0.00 & & \\
\hline WRW \#6 ( $\left.{ }^{\circ} \mathrm{C}\right)$ "WRW \#7 $\left({ }^{\circ} \mathrm{C}\right) "$ & & & & & & & & & & & 0.02 & & & \\
\hline WRW \#6 $\left({ }^{\circ} \mathrm{C}\right)$ "WRW \#8 $\left({ }^{\circ} \mathrm{C}\right) "$ & & & 0.00 & & & & & & & & 0.00 & & & \\
\hline WRW \#6 $\left({ }^{\circ} \mathrm{C}\right)$ "WRW \#12 $\left({ }^{\circ} \mathrm{C}\right) "$ & & & & & & & & & & & & 0.02 & & \\
\hline WRW \#6 $\left({ }^{\circ} \mathrm{C}\right)$ "WRW \#16 $\left({ }^{\circ} \mathrm{C}\right) "$ & & & 0.04 & & & & & & 0.00 & & 0.02 & & & \\
\hline WRW \#6 $\left({ }^{\circ} \mathrm{C}\right)$ "WRW \#17 $\left({ }^{\circ} \mathrm{C}\right) "$ & & 0.01 & 0.00 & & & & & & 0.00 & 0.00 & 0.00 & 0.01 & & \\
\hline WRW \#7 $\left({ }^{\circ} \mathrm{C}\right)$ "WRW \#11 $\left({ }^{\circ} \mathrm{C}\right) "$ & & 0.01 & 0.00 & & & & & & & 0.01 & 0.00 & 0.05 & & \\
\hline WRW \#7 $\left({ }^{\circ} \mathrm{C}\right)$ "WRW \#12 (०C)" & & 0.01 & 0.00 & & & & & & & 0.01 & 0.00 & 0.05 & & \\
\hline WRW \#7 ( $\left.{ }^{\circ} \mathrm{C}\right)$ "WRW \#14 $\left({ }^{\circ} \mathrm{C}\right) "$ & & 0.03 & 0.01 & & & & & & & & 0.01 & & & \\
\hline WRW \#7 $\left({ }^{\circ} \mathrm{C}\right)$ "WRW \#15 $\left({ }^{\circ} \mathrm{C}\right) "$ & & & 0.01 & & & & & & 0.01 & 0.01 & & & & \\
\hline WRW \#7 $\left({ }^{\circ} \mathrm{C}\right)$ "WRW \#18 $\left({ }^{\circ} \mathrm{C}\right) "$ & & & 0.00 & & & & & & & 0.01 & 0.00 & 0.02 & & \\
\hline WRW \#7 $\left({ }^{\circ} \mathrm{C}\right)$ "WRW \#19 $\left({ }^{\circ} \mathrm{C}\right) "$ & & & 0.00 & & & & & & 0.00 & 0.00 & 0.00 & 0.04 & & \\
\hline WRW \#7 $\left({ }^{\circ} \mathrm{C}\right)$ "WRW \#20 $\left({ }^{\circ} \mathrm{C}\right) "$ & & & 0.04 & & & & & & & 0.04 & & & & \\
\hline WRW \#7 $\left({ }^{\circ} \mathrm{C}\right)$ "WRW \#21 $\left({ }^{\circ} \mathrm{C}\right) "$ & & & 0.00 & & & & & & 0.00 & 0.00 & 0.00 & 0.03 & & \\
\hline WRW \#8 $\left({ }^{\circ} \mathrm{C}\right)$ "WRW \#9 $\left({ }^{\circ} \mathrm{C}\right) "$ & & & 0.04 & & & & & & & & & & & \\
\hline WRW \#8 $\left({ }^{\circ} \mathrm{C}\right)$ "WRW \#10 $\left({ }^{\circ} \mathrm{C}\right) "$ & & & 0.01 & & & & & & & & & & & \\
\hline WRW \#8 $\left({ }^{\circ} \mathrm{C}\right)$ "WRW \#11 $\left({ }^{\circ} \mathrm{C}\right) "$ & 0.00 & & 0.00 & & & & 0.00 & & & 0.01 & 0.00 & 0.00 & & \\
\hline WRW \#8 $\left({ }^{\circ} \mathrm{C}\right)$ "WRW \#12 ( $\left.{ }^{\circ} \mathrm{C}\right) "$ & 0.00 & & 0.00 & & & & 0.00 & & & 0.01 & 0.00 & 0.00 & & \\
\hline WRW \#8 $\left({ }^{\circ} \mathrm{C}\right)$ "WRW \#13 $\left({ }^{\circ} \mathrm{C}\right) "$ & & & 0.00 & & & & & & & & 0.01 & & & \\
\hline
\end{tabular}

Figure C2 (cont.). Results of site by site quarterly and monthly comparison using a Kruskal Wallis ANOVA with a post hoc analysis of mean daily Tw collected at twenty-one sites located in West Run Watershed, West Virginia, USA. Only significant $(\alpha=0.05)$ p-value are shown. (Part 2) 


\begin{tabular}{|c|c|c|c|c|c|c|c|c|c|c|c|c|c|c|c|c|}
\hline & Q1 & Q2 & Q3 & Q4 & JAN & FEB & MAR & APR & MAY & JUN & JUL & AUG & SEP & OCT & NOV & DEC \\
\hline & $p$-value & $\mathrm{p}$-value & $\mathrm{p}$-value & $\mathrm{p}$-value & $\mathrm{p}$-value & $\mathrm{p}$-value & $\mathrm{p}$-value & $\mathrm{p}$-value & $\mathrm{p}$-value & $\mathrm{p}$-value & $\mathrm{p}$-value & $\mathrm{p}$-value & $\mathrm{p}$-value & $\mathrm{p}$-value & $\mathrm{p}$-value & $p$-value \\
\hline WRW \#8 ( $\left.{ }^{\circ} \mathrm{C}\right)$ "WRW \#14 $\left({ }^{\circ} \mathrm{C}\right) "$ & & & 0.00 & & & & 0.00 & & & & 0.00 & 0.00 & & & & 0.03 \\
\hline WRW \#8 ( $\left.{ }^{\circ} \mathrm{C}\right)$ "WRW \#15 $\left({ }^{\circ} \mathrm{C}\right) "$ & & & 0.00 & & & & & & & 0.01 & 0.00 & 0.00 & & & & \\
\hline WRW \#8 ( $\left.{ }^{\circ} \mathrm{C}\right)$ "WRW \#16 $\left({ }^{\circ} \mathrm{C}\right) "$ & 0.03 & & & & & & 0.00 & & & & & & & & & \\
\hline WRW \#8 ( $\left.{ }^{\circ} \mathrm{C}\right)$ "WRW \#17 $\left({ }^{\circ} \mathrm{C}\right) "$ & 0.00 & & & & & & 0.00 & & & & & & & & & 0.00 \\
\hline WRW \#8 ( $\left.{ }^{\circ} \mathrm{C}\right)$ "WRW \#18 $\left({ }^{\circ} \mathrm{C}\right) "$ & & & 0.00 & & & & & & & 0.01 & 0.00 & 0.00 & & & & \\
\hline WRW \#8 ( $\left.{ }^{\circ} \mathrm{C}\right)$ "WRW \#19 $\left({ }^{\circ} \mathrm{C}\right) "$ & & & 0.00 & & & & & & & 0.00 & 0.00 & 0.00 & & & & \\
\hline WRW \#8 ( $\left.{ }^{\circ} \mathrm{C}\right)$ "WRW \#20 ( $\left.{ }^{\circ} \mathrm{C}\right) "$ & 0.00 & & 0.00 & & & & 0.00 & & & 0.04 & 0.00 & 0.00 & & & & 0.03 \\
\hline WRW \#8 ( $\left.{ }^{\circ} \mathrm{C}\right)$ "WRW \#21 $\left({ }^{\circ} \mathrm{C}\right) "$ & & & 0.00 & & & & & & 0.05 & 0.00 & 0.00 & 0.00 & & & & \\
\hline 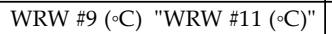 & 0.01 & & 0.01 & & & & 0.00 & & & & & & & & & \\
\hline WRW \#9 $\left({ }^{\circ} \mathrm{C}\right)$ "WRW \#12 $\left({ }^{\circ} \mathrm{C}\right) "$ & 0.01 & & 0.01 & & & & 0.00 & & & & & & & & & \\
\hline WRW \#9 $\left({ }^{\circ} \mathrm{C}\right)$ "WRW \#16 $\left({ }^{\circ} \mathrm{C}\right) "$ & & & & & & & 0.00 & & & & & & & & & \\
\hline WRW \#9 $\left({ }^{\circ} \mathrm{C}\right)$ "WRW \#17 $\left({ }^{\circ} \mathrm{C}\right) "$ & 0.00 & & 0.02 & & & & 0.00 & & 0.00 & & & & & & & 0.03 \\
\hline WRW \#9 $\left({ }^{\circ} \mathrm{C}\right)$ "WRW \#18 $\left({ }^{\circ} \mathrm{C}\right) "$ & & & 0.00 & & & & & & & & 0.01 & & & & & \\
\hline WRW \#9 ( $\left.{ }^{\circ} \mathrm{C}\right)$ "WRW \#19 $\left({ }^{\circ} \mathrm{C}\right) "$ & & & 0.00 & & & & & & & & 0.01 & & & & & \\
\hline WRW \#9 $\left({ }^{\circ} \mathrm{C}\right)$ "WRW \#20 $\left({ }^{\circ} \mathrm{C}\right) "$ & 0.02 & & & & & & 0.00 & & & & & & & & & \\
\hline WRW \#9 ( $\left.{ }^{\circ} \mathrm{C}\right)$ "WRW \#21 $\left({ }^{\circ} \mathrm{C}\right) "$ & & & 0.00 & & & & & & & & 0.01 & & & & & \\
\hline WRW \#10 ( $\left.{ }^{\circ} \mathrm{C}\right)$ "WRW \#17 $\left({ }^{\circ} \mathrm{C}\right) "$ & & & & & & & & & 0.00 & 0.01 & & & & & & \\
\hline WRW \#10 ( $\left.{ }^{\circ} \mathrm{C}\right)$ "WRW \#17 ( $\left.{ }^{\circ} \mathrm{C}\right) "$ & & & 0.00 & & & & & & & & & & & & & \\
\hline WRW \#10 ( $\left.{ }^{\circ} \mathrm{C}\right)$ "WRW \#18 ( $\left.{ }^{\circ} \mathrm{C}\right) "$ & & & 0.00 & & & & & & & & & & & & & \\
\hline WRW \#10 ( $\left.{ }^{\circ} \mathrm{C}\right)$ "WRW \#19 (॰C)" & & & 0.01 & & & & & & & & & & & & & \\
\hline WRW \#10 ( $\left.{ }^{\circ} \mathrm{C}\right) \quad$ WRW \#21 $\left({ }^{\circ} \mathrm{C}\right) "$ & & & 0.01 & & & & & & & & & & & & & \\
\hline WRW \#11 ( $\left.{ }^{\circ} \mathrm{C}\right)$ "WRW \#15 ( $\left.{ }^{\circ} \mathrm{C}\right) "$ & 0.00 & & & & 0.00 & & 0.00 & & & & & & & & & \\
\hline WRW \#11 ( $\left.{ }^{\circ} \mathrm{C}\right)$ "WRW \#16 $\left({ }^{\circ} \mathrm{C}\right) "$ & & & 0.00 & & & & & & 0.01 & 0.01 & 0.00 & 0.01 & & & & \\
\hline WRW \#11 ( $\left.{ }^{\circ} \mathrm{C}\right)$ "WRW \#17 ( $\left.{ }^{\circ} \mathrm{C}\right) "$ & & & 0.00 & & & & & & 0.00 & 0.00 & 0.00 & 0.00 & & & & \\
\hline WRW \#12 ( $\left.{ }^{\circ} \mathrm{C}\right) \quad$ WRW \#15 ( $\left.{ }^{\circ} \mathrm{C}\right) "$ & 0.00 & & & & 0.00 & & 0.00 & & & & & & & & & \\
\hline 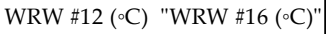 & & & 0.00 & & & & & & 0.01 & 0.01 & 0.00 & 0.01 & & & & \\
\hline WRW \#12( (०C) "WRW \#17 ( $\left.{ }^{\circ} \mathrm{C}\right) "$ & & & 0.00 & & & & & & 0.00 & 0.00 & 0.00 & 0.00 & & & & \\
\hline WRW \#13( (C) "WRW \#17 ( $\left.{ }^{\circ} \mathrm{C}\right) "$ & & & 0.00 & & & & 0.01 & & 0.00 & 0.01 & 0.02 & & & & & \\
\hline WRW \#13 ( $\left.{ }^{\circ} \mathrm{C}\right)$ "WRW \#18 ( $\left.{ }^{\circ} \mathrm{C}\right) "$ & & & 0.02 & & & & & & & & & & & & & \\
\hline WRW \#13 ( $\left.{ }^{\circ} \mathrm{C}\right) \quad$ WRW \#21 $\left({ }^{\circ} \mathrm{C}\right) "$ & & & 0.03 & & & & & & & & & & & & & \\
\hline WRW \#14 ( $\left.{ }^{\circ} \mathrm{C}\right) \quad$ "WRW \#15 ( $\left.{ }^{\circ} \mathrm{C}\right) "$ & 0.01 & & & & & & & & & & & & & & & \\
\hline WRW \#14 ( $\left.{ }^{\circ} \mathrm{C}\right)$ "WRW \#16 $\left({ }^{\circ} \mathrm{C}\right) "$ & & & 0.00 & & & & & & 0.02 & & 0.01 & & & & & \\
\hline WRW \#14 ( $\left.{ }^{\circ} \mathrm{C}\right)$ "WRW \#17 ( $\left.{ }^{\circ} \mathrm{C}\right) "$ & & & 0.00 & & & & & & 0.00 & 0.00 & 0.00 & 0.00 & & & & \\
\hline 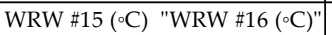 & 0.00 & & 0.00 & & 0.03 & & 0.00 & & 0.00 & 0.01 & & & & & & \\
\hline WRW \#15 ( $\left.{ }^{\circ} \mathrm{C}\right)$ "WRW \#17 ( $\left.{ }^{\circ} \mathrm{C}\right) "$ & 0.00 & 0.00 & 0.00 & 0.02 & & & 0.00 & 0.02 & 0.00 & 0.00 & 0.01 & 0.00 & 0.04 & & 0.03 & 0.01 \\
\hline WRW \#15 ( $\left.{ }^{\circ} \mathrm{C}\right)$ "WRW \#20( $\left.{ }^{\circ} \mathrm{C}\right) "$ & 0.00 & & & & 0.00 & & 0.00 & & & & & & & & & \\
\hline WRW \#15 ( $\left.{ }^{\circ} \mathrm{C}\right) \quad$ WRW \#21 $\left({ }^{\circ} \mathrm{C}\right) "$ & 0.05 & & & & 0.04 & & & & & & & & & & & \\
\hline WRW \#16 ( $\left.{ }^{\circ} \mathrm{C}\right)$ "WRW \#18 ( $\left.{ }^{\circ} \mathrm{C}\right) "$ & & & 0.00 & & & & & & 0.00 & 0.01 & 0.00 & 0.00 & & & & \\
\hline WRW \#16 ( $\left.{ }^{\circ} \mathrm{C}\right) \quad$ WRW \#19 $\left({ }^{\circ} \mathrm{C}\right) "$ & & & 0.00 & & & & & & 0.00 & 0.00 & 0.00 & 0.01 & & & & \\
\hline WRW \#16 ( $\left.{ }^{\circ} \mathrm{C}\right) \quad$ WRW \#20 $\left({ }^{\circ} \mathrm{C}\right) "$ & & & 0.01 & & & & & & 0.02 & & & & & & & \\
\hline WRW \#16 ( $\left.{ }^{\circ} \mathrm{C}\right)$ "WRW \#21 $\left({ }^{\circ} \mathrm{C}\right) "$ & & 0.05 & 0.00 & & & & & & 0.00 & 0.00 & 0.00 & 0.01 & & & & \\
\hline WRW \#17 ( $\left.{ }^{\circ} \mathrm{C}\right)$ "WRW \#18 $\left({ }^{\circ} \mathrm{C}\right) "$ & & 0.00 & 0.00 & & & & 0.00 & & 0.00 & 0.00 & 0.00 & 0.00 & & & & \\
\hline WRW \#17 ( $\left.{ }^{\circ} \mathrm{C}\right)$ "WRW \#19 ( $\left.{ }^{\circ} \mathrm{C}\right) "$ & & 0.00 & 0.00 & & & & & & 0.00 & 0.00 & 0.00 & 0.00 & & & & \\
\hline WRW \#17 ( $\left.{ }^{\circ} \mathrm{C}\right) \quad$ WRW \#20( $\left.{ }^{\circ} \mathrm{C}\right) "$ & & 0.02 & 0.00 & & & & & & 0.00 & 0.00 & & 0.00 & & & & \\
\hline WRW \#17 ( $\left.{ }^{\circ} \mathrm{C}\right)$ "WRW \#21 ( $\left.{ }^{\circ} \mathrm{C}\right) "$ & & 0.00 & 0.00 & & & & & & 0.00 & 0.00 & 0.00 & 0.00 & & & & \\
\hline 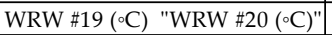 & & & & & & & & & & & 0.00 & & & & & \\
\hline
\end{tabular}

Figure C2 (cont.). Results of site by site quarterly and monthly comparison using a Kruskal Wallis ANOVA with a post hoc analysis of mean daily Tw collected at twenty-one sites located in West Run Watershed, West Virginia, USA. Only significant $(\alpha=0.05)$ p-value are shown. (Part 3) 


\begin{tabular}{|c|c|c|c|c|c|c|c|c|c|c|c|c|c|c|c|c|}
\hline \multirow{2}{*}{ SITE COMPARISON } & Q1 & Q2 & Q3 & Q4 & JAN & FEB & MAR & APR & MAY & JUN & JUL & AUG & SEP & OCT & NOV & DEC \\
\hline & p-value & p-value & $\mathrm{p}$-value & p-value & p-value & p-value & $\mathrm{p}$-value & $\mathrm{p}$-value & p-value & p-value & p-value & $\mathrm{p}$-value & p-value & $\mathrm{p}$-value & p-value & p-value \\
\hline WRW \#1 ( $\left.{ }^{\circ} \mathrm{C}\right)$ "WRW \#2 ('C)" & & & & & & & & & & & & & & & & 0.01 \\
\hline WRW \#1 ( $\left.{ }^{\circ} \mathrm{C}\right)$ "WRW \#3 (C)" & 0.03 & & & & & & 0.00 & & & & & & & & & 0.00 \\
\hline WRW \#1 ( $\left.{ }^{\circ} \mathrm{C}\right)$ "WRW \#4 (ㄷ)" & 0.00 & & 0.00 & 0.01 & 0.00 & & 0.00 & & & & 0.00 & & & & & 0.00 \\
\hline WRW \#1 $\left({ }^{\circ} \mathrm{C}\right)$ "WRW \#5 $\left({ }^{\circ} \mathrm{C}\right) "$ & & 0.00 & 0.00 & 0.04 & 0.00 & & & & 0.00 & 0.00 & 0.00 & 0.00 & 0.00 & & & 0.00 \\
\hline WRW \#1 ( $\left.{ }^{\circ} \mathrm{C}\right)$ "WRW \#6 $\left({ }^{\circ} \mathrm{C}\right) "$ & & 0.00 & 0.00 & & 0.03 & & & & 0.00 & 0.00 & 0.00 & 0.00 & 0.00 & & & 0.00 \\
\hline WRW \#1 ( $\left.{ }^{\circ} \mathrm{C}\right) \quad$ "WRW \#7 $\left({ }^{\circ} \mathrm{C}\right) "$ & & & 0.00 & & 0.02 & & & & & 0.04 & 0.00 & 0.00 & 0.01 & & & \\
\hline WRW \#1 ( $\left.{ }^{\circ} \mathrm{C}\right)$ "WRW \#8 $\left({ }^{\circ} \mathrm{C}\right) "$ & & & 0.00 & & & & & & & & & & & & & \\
\hline WRW \#1 ( $\left.{ }^{\circ} \mathrm{C}\right)$ "WRW \#9 $\left({ }^{\circ} \mathrm{C}\right) "$ & & 0.01 & 0.00 & & & & & & 0.01 & 0.00 & 0.00 & 0.00 & 0.00 & & & \\
\hline WRW \#1 ( $\left.{ }^{\circ} \mathrm{C}\right)$ "WRW \#10 $\left({ }^{\circ} \mathrm{C}\right) "$ & 0.03 & 0.00 & 0.00 & & 0.00 & & & & 0.00 & 0.00 & 0.00 & 0.00 & 0.00 & & & 0.00 \\
\hline WRW \#1 ( $\left.{ }^{\circ} \mathrm{C}\right)$ "WRW \#11 ( $\left.{ }^{\circ} \mathrm{C}\right) "$ & 0.01 & 0.00 & 0.00 & & 0.00 & & & & 0.00 & 0.00 & 0.00 & 0.00 & 0.00 & & & 0.00 \\
\hline WRW \#1 ( $\left.{ }^{\circ} \mathrm{C}\right)$ "WRW \#12 ( $\left.{ }^{\circ} \mathrm{C}\right) "$ & 0.01 & 0.00 & 0.00 & & 0.00 & & & & 0.00 & 0.00 & 0.00 & 0.00 & 0.00 & & & 0.00 \\
\hline WRW \#1 ( $\left.{ }^{\circ} \mathrm{C}\right)$ "WRW \#13 ( $\left.{ }^{\circ} \mathrm{C}\right) "$ & & 0.01 & 0.00 & & 0.00 & & & & 0.00 & 0.00 & 0.00 & 0.00 & 0.00 & & & 0.00 \\
\hline WRW \#1 ( $\left.{ }^{\circ} \mathrm{C}\right) \quad " W R W \# 14\left({ }^{\circ} \mathrm{C}\right) "$ & 0.00 & 0.00 & 0.00 & & 0.00 & & & & 0.00 & 0.00 & 0.00 & 0.00 & 0.00 & & & 0.00 \\
\hline WRW \#1 ( $\left.{ }^{\circ} \mathrm{C}\right)$ "WRW \#15 ( $\left.{ }^{\circ} \mathrm{C}\right) "$ & & 0.00 & 0.00 & & & & & & 0.00 & 0.00 & 0.00 & 0.00 & 0.00 & & & \\
\hline WRW \#1 ( $\left.{ }^{\circ} \mathrm{C}\right)$ "WRW \#16 ( $\left.{ }^{\circ} \mathrm{C}\right) "$ & 0.00 & & 0.00 & & 0.00 & & 0.04 & & & & 0.00 & 0.00 & & & & 0.00 \\
\hline WRW \#1 ( $\left.{ }^{\circ} \mathrm{C}\right)$ "WRW \#17 ( $\left.{ }^{\circ} \mathrm{C}\right) "$ & 0.00 & & 0.00 & 0.00 & 0.00 & & 0.00 & & & 0.00 & & & & & & 0.00 \\
\hline WRW \#1 ( $\left.{ }^{\circ} \mathrm{C}\right)$ "WRW \#18 ( $\left.{ }^{\circ} \mathrm{C}\right) "$ & & 0.00 & 0.00 & & 0.01 & & & & 0.00 & & 0.00 & 0.00 & 0.00 & & & 0.00 \\
\hline WRW \#1 ( $\left.{ }^{\circ} \mathrm{C}\right) \quad " W R W \# 19\left({ }^{\circ} \mathrm{C}\right) "$ & 0.01 & 0.00 & 0.00 & & 0.00 & & & & 0.00 & 0.00 & 0.00 & 0.00 & 0.00 & & & 0.00 \\
\hline WRW \#1 ( $\left.{ }^{\circ} \mathrm{C}\right)$ "WRW \#20 ( $\left.{ }^{\circ} \mathrm{C}\right) "$ & 0.00 & & 0.00 & & 0.00 & & 0.00 & & & 0.00 & 0.00 & 0.00 & 0.00 & & & 0.00 \\
\hline WRW \#1 ( $\left.{ }^{\circ} \mathrm{C}\right)$ "WRW \#21 ( $\left.{ }^{\circ} \mathrm{C}\right) "$ & 0.00 & 0.00 & 0.00 & & 0.00 & & & & 0.00 & 0.00 & 0.00 & 0.00 & 0.00 & & 0.04 & 0.00 \\
\hline WRW \#2 ( $\left.{ }^{\circ} \mathrm{C}\right)$ "WRW \#4 (C)" & & & 0.00 & & & & & & & & 0.00 & & & & & \\
\hline WRW \#2 ( $\left.{ }^{\circ} \mathrm{C}\right)$ "WRW \#5 (C)" & & 0.00 & 0.00 & & & & & & 0.00 & 0.00 & 0.00 & 0.00 & 0.00 & & & \\
\hline WRW \#2 ( $\left.{ }^{\circ} \mathrm{C}\right)$ "WRW \#6 (C)" & & 0.00 & 0.00 & & & & & & 0.00 & 0.00 & 0.00 & 0.00 & 0.00 & & & \\
\hline WRW \#2 ( $\left.{ }^{\circ} \mathrm{C}\right)$ "WRW \#7 (C)" & & & 0.00 & & & & & & & & 0.00 & 0.00 & 0.00 & & & \\
\hline WRW \#2 ( $\left.{ }^{\circ} \mathrm{C}\right)$ "WRW \#8 ( $\left.{ }^{\circ} \mathrm{C}\right) "$ & & & 0.03 & & & & & & & & & & & & & \\
\hline WRW \#2 ( $\left.{ }^{\circ} \mathrm{C}\right)$ "WRW \#9 (॰C)" & & 0.05 & 0.00 & & & & & & 0.01 & 0.04 & 0.00 & 0.00 & 0.00 & & & \\
\hline WRW \#2 ( $\left.{ }^{\circ} \mathrm{C}\right) \quad " W R W \# 10\left({ }^{\circ} \mathrm{C}\right) "$ & & 0.00 & 0.00 & & & & & & 0.00 & 0.00 & 0.00 & 0.00 & 0.00 & & & \\
\hline WRW \#2 ( $\left.{ }^{\circ} \mathrm{C}\right)$ "WRW \#11 ( $\left.{ }^{\circ} \mathrm{C}\right) "$ & & 0.00 & 0.00 & & & & & & 0.00 & 0.00 & 0.00 & 0.00 & 0.00 & & & \\
\hline WRW \#2 ( $\left.{ }^{\circ} \mathrm{C}\right) \quad " W R W \# 12\left({ }^{\circ} \mathrm{C}\right) "$ & & 0.00 & 0.00 & & & & & & 0.00 & 0.00 & 0.00 & 0.00 & 0.00 & & & \\
\hline WRW \#2 ( $\left.{ }^{\circ} \mathrm{C}\right) \quad " W R W \# 13\left({ }^{\circ} \mathrm{C}\right) "$ & & 0.02 & 0.00 & & & & & & 0.00 & 0.00 & 0.00 & 0.00 & 0.00 & & & \\
\hline WRW \#2 ( $\left.{ }^{\circ} \mathrm{C}\right) \quad$ "WRW \#14 ( $\left.{ }^{\circ} \mathrm{C}\right) "$ & & 0.00 & 0.00 & & & & & & 0.00 & 0.00 & 0.00 & 0.00 & 0.00 & & & \\
\hline WRW \#2 ( $\left.{ }^{\circ} \mathrm{C}\right)$ "WRW \#15 ( $\left.{ }^{\circ} \mathrm{C}\right) "$ & & 0.00 & 0.00 & & & & & & 0.00 & 0.00 & 0.00 & 0.00 & 0.00 & & & \\
\hline WRW \#2 ( $\left.{ }^{\circ} \mathrm{C}\right) \quad " W R W \# 16\left({ }^{\circ} \mathrm{C}\right) "$ & & & 0.00 & & & & & & & & 0.00 & 0.00 & 0.03 & & & \\
\hline WRW \#2 ( $\left.{ }^{\circ} \mathrm{C}\right) \quad " W R W \# 17\left({ }^{\circ} \mathrm{C}\right) "$ & & & 0.03 & & & & & & & & & & & & & \\
\hline WRW \#2 ( $\left.{ }^{\circ} \mathrm{C}\right) \quad " W R W \# 18\left({ }^{\circ} \mathrm{C}\right) "$ & & 0.00 & 0.00 & & & & & & 0.00 & 0.00 & 0.00 & 0.00 & 0.00 & & & \\
\hline WRW \#2 ( $\left.{ }^{\circ} \mathrm{C}\right)$ "WRW \#19 (C)" & & 0.00 & 0.00 & & & & & & 0.00 & 0.00 & 0.00 & 0.00 & 0.00 & & & \\
\hline WRW \#2 ( $\left.{ }^{\circ} \mathrm{C}\right)$ "WRW \#20 (॰C)" & & & 0.00 & & 0.04 & & & & & 0.00 & 0.00 & 0.00 & 0.00 & & & \\
\hline WRW \#2 ( $\left.{ }^{\circ} \mathrm{C}\right) \quad$ "WRW \#21 ( $\left.{ }^{\circ} \mathrm{C}\right) "$ & & 0.00 & 0.00 & & & & & & 0.00 & 0.00 & 0.00 & 0.00 & 0.00 & & & \\
\hline WRW \#3 (ㄷ) "WRW \#5 (॰C)" & & 0.00 & 0.00 & & & & & & 0.00 & 0.00 & 0.00 & 0.00 & 0.01 & & & \\
\hline WRW \#3 ( $\left.{ }^{\circ} \mathrm{C}\right)$ "WRW \#6 ( $\left.{ }^{\circ} \mathrm{C}\right) "$ & & 0.02 & 0.00 & & & & & & 0.00 & 0.00 & 0.00 & 0.00 & & & & \\
\hline WRW \#3 ( $\left.{ }^{\circ} \mathrm{C}\right)$ "WRW \#7 (C)" & & & 0.00 & & & & & & & & & & & & & \\
\hline WRW \#3 ( $\left.{ }^{\circ} \mathrm{C}\right)$ "WRW \#9 $\left({ }^{\circ} \mathrm{C}\right) "$ & & & 0.00 & & & & & & & & & 0.03 & & & & \\
\hline WRW \#3 ( $\left.{ }^{\circ} \mathrm{C}\right)$ "WRW \#10 (०C)" & & & 0.00 & & & & & & & & & 0.02 & & & & \\
\hline WRW \#3 ( $\left.{ }^{\circ} \mathrm{C}\right)$ "WRW \#11 ( $\left.{ }^{\circ} \mathrm{C}\right) "$ & & 0.00 & 0.00 & & & & & & 0.00 & 0.00 & 0.00 & 0.00 & 0.00 & & & \\
\hline WRW \#3 (C) "WRW \#12 (॰C)" & & 0.00 & 0.00 & & & & & & 0.00 & 0.00 & 0.00 & 0.00 & 0.00 & & & \\
\hline WRW \#3 ( $\left.{ }^{\circ} \mathrm{C}\right)$ "WRW \#13 (C)" & & & 0.00 & & & & & & & & 0.00 & 0.00 & & & & \\
\hline
\end{tabular}

Figure C3. Results of site by site quarterly and monthly comparison using a Kruskal Wallis

ANOVA with a post hoc analysis of maximum daily Tw collected at twenty-one sites located in

West Run Watershed, West Virginia, USA. Only significant $(\alpha=0.05)$-value are shown. (Part 1) 


\begin{tabular}{|c|c|c|c|c|c|c|c|c|c|c|c|c|c|c|c|c|}
\hline \multirow{2}{*}{ SITE COMPARISON } & Q1 & Q2 & Q3 & $\mathrm{Q4}$ & JAN & FEB & MAR & APR & MAY & JUN & JUL & AUG & SEP & OCT & NOV & DEC \\
\hline & $p$-value & $p$-value & $\mathrm{p}$-value & $\mathrm{p}$-value & $\mathrm{p}$-value & p-value & $\mathrm{p}$-value & $p$-value & $\mathrm{p}$-value & $\mathrm{p}$-value & p-value & p-value & $\mathrm{p}$-value & $\mathrm{p}$-value & p-value & $\mathrm{p}$-value \\
\hline 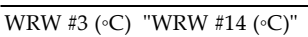 & & 0.00 & 0.00 & & & & & & 0.00 & 0.00 & 0.00 & 0.00 & 0.02 & & & \\
\hline WRW \#3 (ㄷ) "WRW \#15 (ㄷ)" & & 0.02 & 0.00 & & & & & & 0.00 & 0.01 & 0.01 & 0.00 & 0.00 & & & \\
\hline WRW \#3 $\left({ }^{\circ} \mathrm{C}\right)$ "WRW \#16 $\left({ }^{\circ} \mathrm{C}\right) "$ & & & 0.00 & & & & & & & & & & & & & \\
\hline WRW \#3 (ㄷ) "WRW \#18 (ㄷ)" & & & 0.00 & & & & & & & 0.01 & 0.00 & 0.00 & 0.02 & & & \\
\hline WRW \#3 (C) "WRW \#19 (ㄷ)" & & 0.03 & 0.00 & & & & & & 0.01 & 0.00 & 0.00 & 0.00 & & & & \\
\hline WRW \#3 (ㄷ) "WRW \#20 ( $\left.{ }^{\circ} \mathrm{C}\right) "$ & & & 0.00 & & & & & & & & & 0.03 & & & & \\
\hline WRW \#3 ('C) "WRW \#21 ( $\left.{ }^{\circ} \mathrm{C}\right) "$ & & 0.03 & 0.00 & & & & & & 0.01 & 0.00 & 0.00 & 0.00 & 0.03 & & & \\
\hline WRW \#4 (ㄷ) "WRW \#5 (०C)" & & 0.00 & 0.00 & & & & & & 0.00 & 0.00 & 0.00 & 0.00 & & & & \\
\hline WRW \#4 (C) "WRW \#6 (ㄷ)" & & 0.02 & 0.00 & & & & & & 0.00 & & & & & & & \\
\hline 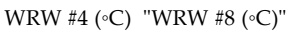 & & & & & & & 0.02 & & & & & & & & & 0.03 \\
\hline WRW \#4 $\left({ }^{\circ} \mathrm{C}\right) \quad$ "WRW \#10 $\left({ }^{\circ} \mathrm{C}\right) "$ & & & & & & & & & 0.04 & & & & & & & \\
\hline WRW \#4 (ㄷ) "WRW \#11 (ㄷ)" & & 0.00 & 0.00 & & & & & & 0.00 & 0.00 & 0.00 & 0.00 & & & & \\
\hline WRW \#4 (ㄷ) "WRW \#12 (ㄷ)" & & 0.00 & 0.00 & & & & & & 0.00 & 0.00 & 0.00 & 0.00 & & & & \\
\hline WRW \#4 (ㄷ) "WRW \#13 (ㄷ)" & & & 0.04 & & & & & & & & & & & & & \\
\hline WRW \#4 (ㄷ) "WRW \#14 (ㄷ)" & & 0.00 & 0.00 & & & & & & 0.00 & 0.00 & 0.00 & 0.00 & & & & \\
\hline WRW \#4 (ㄷ) "WRW \#15 (ㄷ)" & 0.03 & 0.02 & 0.00 & & & & 0.04 & & 0.00 & & & 0.01 & & & & \\
\hline WRW \#4 (ㄷ) "WRW \#18 (ㄷ)" & & & 0.00 & & & & & & & & 0.03 & 0.00 & & & & \\
\hline WRW \#4 $\left({ }^{\circ} \mathrm{C}\right) \quad$ "WRW \#19 $\left({ }^{\circ} \mathrm{C}\right) "$ & & 0.04 & 0.00 & & & & & & 0.00 & 0.02 & 0.00 & 0.00 & & & & \\
\hline WRW \#4 (०C) "WRW \#21 (ㄷ)" & & 0.03 & 0.00 & & & & & & 0.00 & 0.03 & 0.00 & 0.00 & & & & \\
\hline WRW \#5 ( $\left.{ }^{\circ} \mathrm{C}\right)$ "WRW \#7 ( $\left.{ }^{\circ} \mathrm{C}\right) "$ & & 0.02 & 0.00 & & & & & & 0.00 & 0.00 & 0.00 & 0.04 & & & & \\
\hline WRW \#5 ( $\left.{ }^{\circ} \mathrm{C}\right)$ "WRW \#8 $\left({ }^{\circ} \mathrm{C}\right) "$ & & 0.02 & 0.00 & & & & & & 0.00 & 0.00 & 0.00 & 0.00 & & & & 0.02 \\
\hline WRW \#5 ( $\left.{ }^{\circ} \mathrm{C}\right)$ "WRW \#9 $\left({ }^{\circ} \mathrm{C}\right) "$ & & & 0.00 & & & & & & & 0.00 & 0.00 & & & & & \\
\hline WRW \#5 (ㄷ) "WRW \#10 ( $\left.{ }^{\circ} \mathrm{C}\right) "$ & & & 0.00 & & & & & & & & 0.00 & & & & & \\
\hline WRW \#5 $\left({ }^{\circ} \mathrm{C}\right) \quad$ "WRW \#13 $\left({ }^{\circ} \mathrm{C}\right) "$ & & & 0.01 & & & & & & & & 0.01 & & & & & \\
\hline 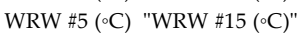 & & & & & & & & & & & 0.00 & & & & & \\
\hline WRW \#5 $\left({ }^{\circ} \mathrm{C}\right) \quad$ "WRW \#16 $\left({ }^{\circ} \mathrm{C}\right) "$ & & 0.00 & 0.00 & & & & & & 0.00 & 0.00 & 0.00 & 0.01 & & & & \\
\hline WRW \#5 (ㄷ) "WRW \#17 (ㄷ)" & & 0.00 & 0.00 & & & & & & 0.00 & 0.00 & 0.00 & 0.00 & 0.00 & & & \\
\hline WRW \#5 $\left({ }^{\circ} \mathrm{C}\right) \quad$ "WRW \#20 (ㄷ)" & & & 0.00 & & & & & & 0.01 & & 0.00 & & & & & \\
\hline WRW \#6 ( $\left.{ }^{\circ} \mathrm{C}\right)$ "WRW \#8 $\left({ }^{\circ} \mathrm{C}\right) "$ & & & 0.00 & & & & & & & & 0.00 & 0.01 & & & & \\
\hline WRW \#6 ('C) "WRW \#11 ( $\left.{ }^{\circ} \mathrm{C}\right) "$ & & & 0.00 & & & & & & & & & 0.00 & & & & \\
\hline WRW \#6 $\left({ }^{\circ} \mathrm{C}\right) \quad$ "WRW \#12 $\left({ }^{\circ} \mathrm{C}\right) "$ & & & 0.00 & & & & & & & & & 0.00 & & & & \\
\hline WRW \#6 $\left({ }^{\circ} \mathrm{C}\right) \quad$ "WRW \#16 $\left({ }^{\circ} \mathrm{C}\right) "$ & & & & & & & & & 0.02 & 0.02 & & & & & & \\
\hline WRW \#6 (ㄷ) "WRW \#17 (ㄷ)" & & 0.00 & 0.00 & & & & & & 0.00 & 0.00 & 0.00 & & & & & \\
\hline WRW \#7 ( $\left.{ }^{\circ} \mathrm{C}\right)$ "WRW \#8 $\left({ }^{\circ} \mathrm{C}\right) "$ & & & 0.02 & & & & & & & & & & & & & \\
\hline WRW \#7 (ㄷ) "WRW \#11 ( $\left.{ }^{\circ} \mathrm{C}\right) "$ & & 0.00 & 0.00 & & & & & & 0.00 & 0.00 & 0.00 & 0.00 & & & & \\
\hline WRW \#7 (ㄷ) "WRW \#12 (ㄷ)" & & 0.00 & 0.00 & & & & & & 0.00 & 0.00 & 0.00 & 0.00 & & & & \\
\hline WRW \#7 (०C) "WRW \#14 (ㄷ)" & & & 0.00 & & & & & & & & 0.00 & & & & & \\
\hline WRW \#7 (०C) "WRW \#17 (ㄷ)" & & & 0.02 & & & & & & & & & & & & & \\
\hline WRW \#7 (०C) "WRW \#18 (ㄷ)" & & & 0.00 & & & & & & & & 0.04 & & & & & \\
\hline WRW \#7 (०C) "WRW \#19 (ㄷ)" & & & 0.00 & & & & & & & & 0.00 & & & & & \\
\hline WRW \#7 (०C) "WRW \#21 (ㄷ)" & & & 0.00 & & & & & & & & 0.00 & & & & & \\
\hline WRW \#8 ( $\left.{ }^{\circ} \mathrm{C}\right) \quad$ "WRW \#9 $\left({ }^{\circ} \mathrm{C}\right) "$ & & & 0.00 & & & & & & & & & & & & & \\
\hline WRW \#8 (०C) "WRW \#10 (ㄷ)" & & & 0.00 & & & & & & & & & & & & & \\
\hline WRW \#8 (॰C) "WRW \#11 (०C)" & & 0.00 & 0.00 & & & & & & 0.00 & 0.00 & 0.00 & 0.00 & 0.02 & & & \\
\hline WRW \#8 ('C) "WRW \#12 (ㄷ)" & & 0.00 & 0.00 & & & & & & 0.00 & 0.00 & 0.00 & 0.00 & 0.02 & & & \\
\hline 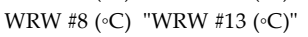 & & & 0.00 & & & & & & & & 0.00 & 0.00 & & & & \\
\hline WRW \#8 $\left({ }^{\circ} \mathrm{C}\right) \quad$ WRW \#14 $\left({ }^{\circ} \mathrm{C}\right) "$ & & & 0.00 & & & & & & & 0.00 & 0.00 & 0.00 & & & & 0.05 \\
\hline WRW \#8 (ㄷ) "WRW \#15 (ㄷ)" & & & 0.00 & & & & & & & & 0.02 & 0.00 & 0.05 & & & \\
\hline
\end{tabular}

Figure C3 (cont.). Results of site by site quarterly and monthly comparison using a Kruskal Wallis ANOVA with a post hoc analysis of maximum daily Tw collected at twenty-one sites located in West Run Watershed, West Virginia, USA. Only significant $(\alpha=0.05)$ p-value are shown. (Part 2) 


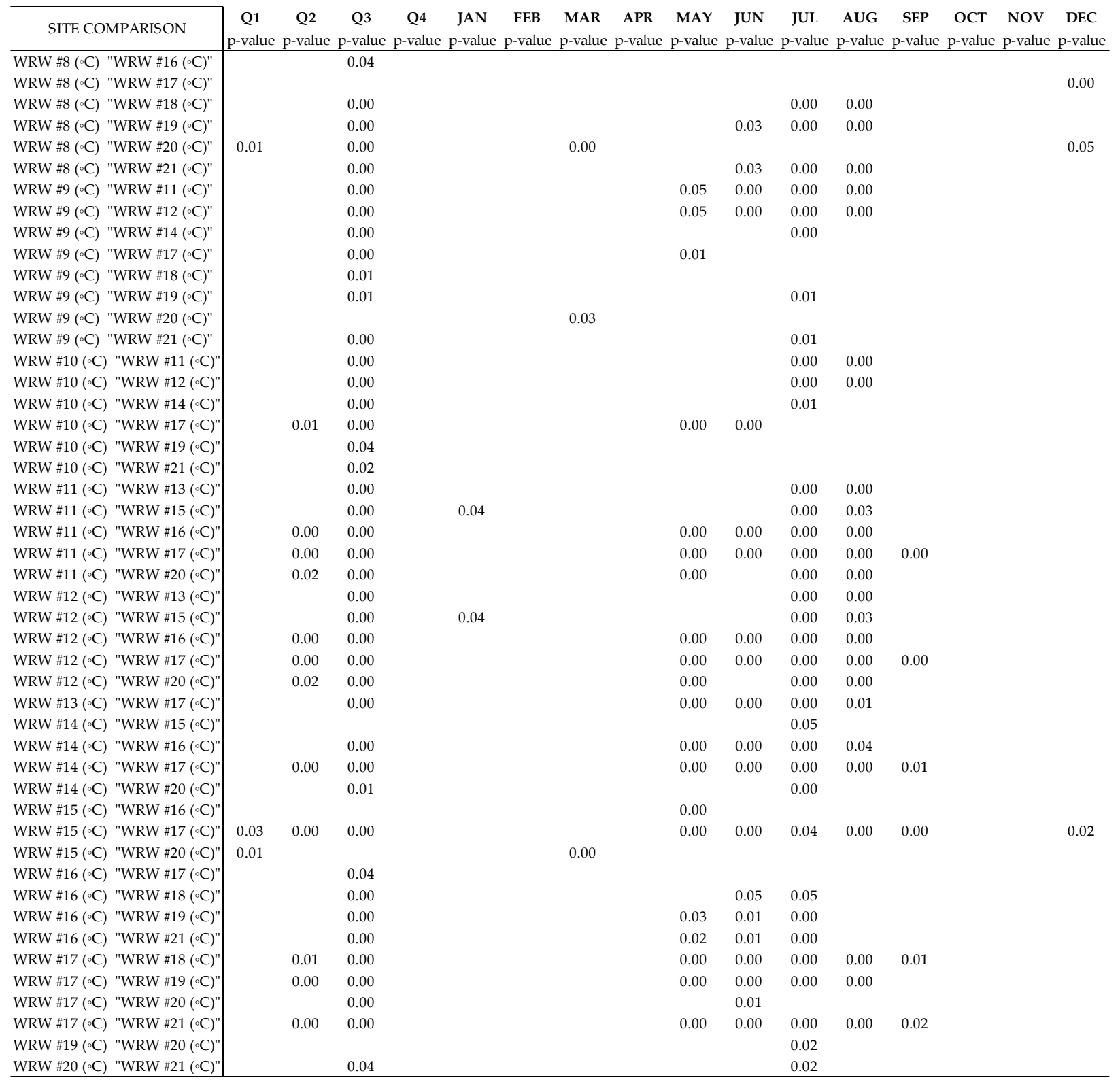

Figure C3 (cont.). Results of site by site quarterly and monthly comparison using a Kruskal Wallis ANOVA with a post hoc analysis of maximum daily Tw collected at twenty-one sites located in West Run Watershed, West Virginia, USA. Only significant $(\alpha=0.05)$ p-value are shown. (Part 3) 


\begin{tabular}{|c|c|c|c|c|c|c|c|c|c|c|c|c|c|c|c|c|}
\hline & Q1 & Q2 & Q3 & Q4 & JAN & FEB & MAR & APR & MAY & JUN & JUL & AUG & SEP & OCT & NOV & DEC \\
\hline & p-value & $\mathrm{p}$-value & $\mathrm{p}$-value & $\mathrm{p}$-value & p-value & $\mathrm{p}$-value & $\mathrm{p}$-value & $\mathrm{p}$-value & $\mathrm{p}$-value & $\mathrm{p}$-value & $\mathrm{p}$-value & $\mathrm{p}$-value & p-value & $\mathrm{p}$-value & $\mathrm{p}$-value & $\mathrm{p}$-value \\
\hline WRW \#1 (ㄷ) "WRW \#2 ('C)" & & & & & & & & & & & & & & & & 0.02 \\
\hline WRW \#1 ( $\left.{ }^{\circ} \mathrm{C}\right)$ "WRW \#3 $\left({ }^{\circ} \mathrm{C}\right) "$ & 0.01 & & 0.00 & & & & & & & & & & & & & 0.00 \\
\hline WRW \#1 ( $\left.{ }^{\circ} \mathrm{C}\right)$ "WRW \#4 $\left({ }^{\circ} \mathrm{C}\right) "$ & 0.00 & & 0.00 & 0.00 & 0.00 & & 0.00 & 0.01 & & & 0.04 & & & & 0.05 & 0.00 \\
\hline WRW \#1 (C) "WRW \#5 (॰C)" & 0.00 & 0.00 & 0.00 & 0.00 & 0.00 & & 0.00 & & 0.00 & 0.00 & 0.00 & 0.00 & 0.00 & & 0.00 & 0.00 \\
\hline WRW \#1 (ㄷ) "WRW \#6 (ㄷ)" & 0.00 & & 0.00 & & 0.01 & & 0.00 & & & 0.00 & 0.00 & 0.00 & 0.00 & & & 0.00 \\
\hline WRW \#1 ( $\left.{ }^{\circ} \mathrm{C}\right)$ "WRW \#7 (०C)" & 0.00 & & 0.00 & & 0.00 & & 0.00 & & & & 0.00 & 0.00 & 0.00 & & & 0.00 \\
\hline WRW \#1 (ㄷ) "WRW \#8 (॰C)" & & & 0.00 & & 0.02 & & & & & & 0.00 & 0.00 & 0.00 & & & \\
\hline WRW \#1 ( $\left.{ }^{\circ} \mathrm{C}\right)$ "WRW \#9 (॰C)" & 0.04 & & 0.00 & & & & & & & 0.00 & 0.00 & 0.00 & 0.00 & & & \\
\hline WRW \#1 ( $\left.{ }^{\circ} \mathrm{C}\right)$ "WRW \#10 $\left({ }^{\circ} \mathrm{C}\right) "$ & 0.00 & & 0.00 & 0.00 & 0.00 & & 0.00 & & & 0.00 & 0.00 & 0.00 & 0.00 & & 0.02 & 0.00 \\
\hline WRW \#1 ( $\left.{ }^{\circ} \mathrm{C}\right)$ "WRW \#11 ( $\left.{ }^{\circ} \mathrm{C}\right) "$ & 0.00 & & 0.00 & 0.00 & 0.00 & & 0.00 & 0.00 & & 0.00 & 0.00 & 0.00 & 0.00 & & 0.00 & 0.00 \\
\hline WRW \#1 (C) "WRW \#12 (॰C)" & 0.00 & & 0.00 & 0.00 & 0.00 & & 0.00 & 0.00 & & 0.00 & 0.00 & 0.00 & 0.00 & & 0.00 & 0.00 \\
\hline WRW \#1 (C) "WRW \#13 (॰C)" & 0.00 & & 0.00 & 0.05 & 0.00 & & 0.01 & & & 0.00 & 0.00 & 0.00 & 0.00 & & & 0.00 \\
\hline WRW \#1 (C) "WRW \#14 (॰C)" & 0.00 & & 0.00 & 0.00 & 0.00 & & 0.00 & & & 0.00 & 0.00 & 0.00 & 0.00 & & 0.02 & 0.00 \\
\hline WRW \#1 ( $\left.{ }^{\circ} \mathrm{C}\right)$ "WRW \#15 (॰C)" & & 0.02 & 0.00 & & & & & & 0.00 & 0.00 & 0.00 & 0.00 & 0.00 & & & \\
\hline WRW \#1 ( $\left.{ }^{\circ} \mathrm{C}\right) \quad$ "WRW \#16 ( $\left.{ }^{\circ} \mathrm{C}\right) "$ & 0.00 & & 0.00 & 0.00 & 0.00 & & 0.00 & 0.02 & & 0.00 & 0.00 & 0.00 & 0.00 & & & 0.00 \\
\hline WRW \#1 ( $\left.{ }^{\circ} \mathrm{C}\right)$ "WRW \#17 ( $\left.{ }^{\circ} \mathrm{C}\right) "$ & 0.00 & & 0.00 & 0.00 & 0.00 & & 0.00 & 0.00 & & 0.00 & 0.00 & 0.00 & 0.00 & & 0.00 & 0.00 \\
\hline WRW \#1 ( $\left.{ }^{\circ} \mathrm{C}\right)$ "WRW \#18 ( $\left.{ }^{\circ} \mathrm{C}\right) "$ & 0.00 & & 0.00 & & 0.01 & & 0.00 & & 0.03 & 0.00 & 0.00 & 0.00 & 0.00 & & & 0.00 \\
\hline WRW \#1 (C) "WRW \#19 (॰C)" & 0.00 & & 0.00 & 0.01 & 0.00 & & 0.00 & & 0.00 & 0.00 & 0.00 & 0.00 & 0.00 & & 0.02 & 0.00 \\
\hline WRW \#1 ( $\left.{ }^{\circ} \mathrm{C}\right)$ "WRW \#20 ( $\left.{ }^{\circ} \mathrm{C}\right) "$ & 0.00 & & 0.00 & 0.00 & 0.00 & & 0.00 & & 0.00 & & 0.00 & 0.00 & 0.00 & & 0.00 & 0.00 \\
\hline WRW \#1 ( $\left.{ }^{\circ} \mathrm{C}\right)$ "WRW \#21 $\left({ }^{\circ} \mathrm{C}\right) "$ & 0.00 & 0.03 & 0.00 & 0.00 & 0.00 & & 0.00 & & 0.00 & & 0.00 & 0.00 & 0.00 & & 0.01 & 0.00 \\
\hline WRW \#2 (ㄷ) "WRW \#4 ('C)" & 0.00 & & 0.02 & & 0.00 & & & & & & & & & & & \\
\hline WRW \#2 ( $\left.{ }^{\circ} \mathrm{C}\right)$ "WRW \#5 (ㄷ)" & & 0.00 & 0.00 & & & & & & 0.00 & 0.00 & 0.00 & 0.00 & 0.00 & & & \\
\hline WRW \#2 ( $\left.{ }^{\circ} \mathrm{C}\right)$ "WRW \#6 (ㄷ)" & & & 0.00 & & & & & & & 0.00 & 0.00 & 0.00 & 0.00 & & & \\
\hline WRW \#2 (ㄷ) "WRW \#7 (॰C)" & & & 0.00 & & 0.00 & & & & & & 0.02 & 0.00 & 0.01 & & & \\
\hline WRW \#2 ( $\left.{ }^{\circ} \mathrm{C}\right)$ "WRW \#8 (C)" & & & 0.00 & & & & & & & & & & 0.05 & & & \\
\hline WRW \#2 ( $\left.{ }^{\circ} \mathrm{C}\right)$ "WRW \#9 (ㄷ)" & & & 0.00 & & & & & & & & 0.00 & 0.00 & 0.00 & & & \\
\hline WRW \#2 ( $\left.{ }^{\circ} \mathrm{C}\right)$ "WRW \#10 ( $\left.{ }^{\circ} \mathrm{C}\right) "$ & & & 0.00 & & 0.01 & & & & & & 0.00 & 0.00 & 0.01 & & & \\
\hline WRW \#2 ( $\left.{ }^{\circ} \mathrm{C}\right)$ "WRW \#11 ( $\left.{ }^{\circ} \mathrm{C}\right) "$ & 0.00 & & 0.00 & & 0.00 & & 0.00 & & & & 0.00 & 0.00 & 0.02 & & & \\
\hline WRW \#2 (॰C) "WRW \#12 (॰C)" & 0.00 & & 0.00 & & 0.00 & & 0.00 & & & & 0.00 & 0.00 & 0.02 & & & \\
\hline WRW \#2 ( $\left.{ }^{\circ} \mathrm{C}\right)$ "WRW \#13 (॰C)" & & & 0.00 & & 0.00 & & & & & & 0.00 & 0.00 & 0.04 & & & \\
\hline WRW \#2 ( $\left.{ }^{\circ} \mathrm{C}\right)$ "WRW \#14 (॰C)" & 0.01 & & 0.00 & & & & & & & & 0.00 & 0.00 & 0.00 & & & \\
\hline WRW \#2 ( $\left.{ }^{\circ} \mathrm{C}\right)$ "WRW \#15 (॰C)" & & & 0.00 & & & & & & 0.01 & 0.00 & 0.00 & 0.00 & 0.00 & & & \\
\hline WRW \#2 ( $\left.{ }^{\circ} \mathrm{C}\right)$ "WRW \#16 $\left({ }^{\circ} \mathrm{C}\right) "$ & 0.00 & & 0.00 & & 0.00 & & & & & & 0.00 & 0.00 & 0.01 & & & \\
\hline WRW \#2 ( $\left.{ }^{\circ} \mathrm{C}\right)$ "WRW \#17 (॰C)" & 0.00 & & 0.00 & & 0.02 & & 0.00 & & & & 0.04 & & & & & \\
\hline WRW \#2 (॰C) "WRW \#18 (॰C)" & & & 0.00 & & & & & & & 0.00 & 0.00 & 0.00 & 0.00 & & & \\
\hline WRW \#2 ( $\left.{ }^{\circ} \mathrm{C}\right)$ "WRW \#19 (०C)" & & & 0.00 & & 0.00 & & & & 0.01 & 0.00 & 0.00 & 0.00 & 0.00 & & & \\
\hline WRW \#2 ( $\left.{ }^{\circ} \mathrm{C}\right)$ "WRW \#20 ( $\left.{ }^{\circ} \mathrm{C}\right) "$ & 0.00 & & 0.00 & & 0.00 & & & & 0.01 & 0.00 & 0.00 & 0.00 & 0.00 & & & \\
\hline WRW \#2 ( $\left.{ }^{\circ} \mathrm{C}\right)$ "WRW \#21 ( $\left.{ }^{\circ} \mathrm{C}\right) "$ & 0.01 & & 0.00 & & 0.00 & & & & 0.00 & 0.00 & 0.00 & 0.00 & 0.00 & & & \\
\hline WRW \#3 (C) "WRW \#4 (C)" & & & & & 0.00 & & & & & & & & & & & \\
\hline WRW \#3 (ㄷ) "WRW \#5 (॰C)" & & & 0.00 & & & & & & & 0.00 & 0.00 & 0.00 & 0.02 & & & \\
\hline WRW \#3 (C) "WRW \#6 (॰C)" & & & 0.00 & & & & & & & & 0.00 & 0.00 & & & & \\
\hline WRW \#3 (C) "WRW \#7 (॰C)" & & & 0.00 & & & & & & & & & & & & & \\
\hline WRW \#3 (ㄷ) "WRW \#9 (॰C)" & & & 0.00 & & & & & & & & & & & & & \\
\hline WRW \#3 ( $\left.{ }^{\circ} \mathrm{C}\right) \quad$ "WRW \#10 ( $\left.{ }^{\circ} \mathrm{C}\right) "$ & & & 0.00 & & & & & & & & 0.01 & 0.04 & & & & \\
\hline WRW \#3 (C) "WRW \#11 (॰C)" & 0.00 & & 0.00 & & 0.00 & & 0.00 & & & & 0.00 & 0.01 & & & & \\
\hline
\end{tabular}

Figure C4. Results of site by site quarterly and monthly comparison using a Kruskal Wallis

ANOVA with a post hoc analysis of minimum daily Tw collected at twenty-one sites located in

West Run Watershed, West Virginia, USA. Only significant $(\alpha=0.05)$ p-value are shown. (Part 1) 


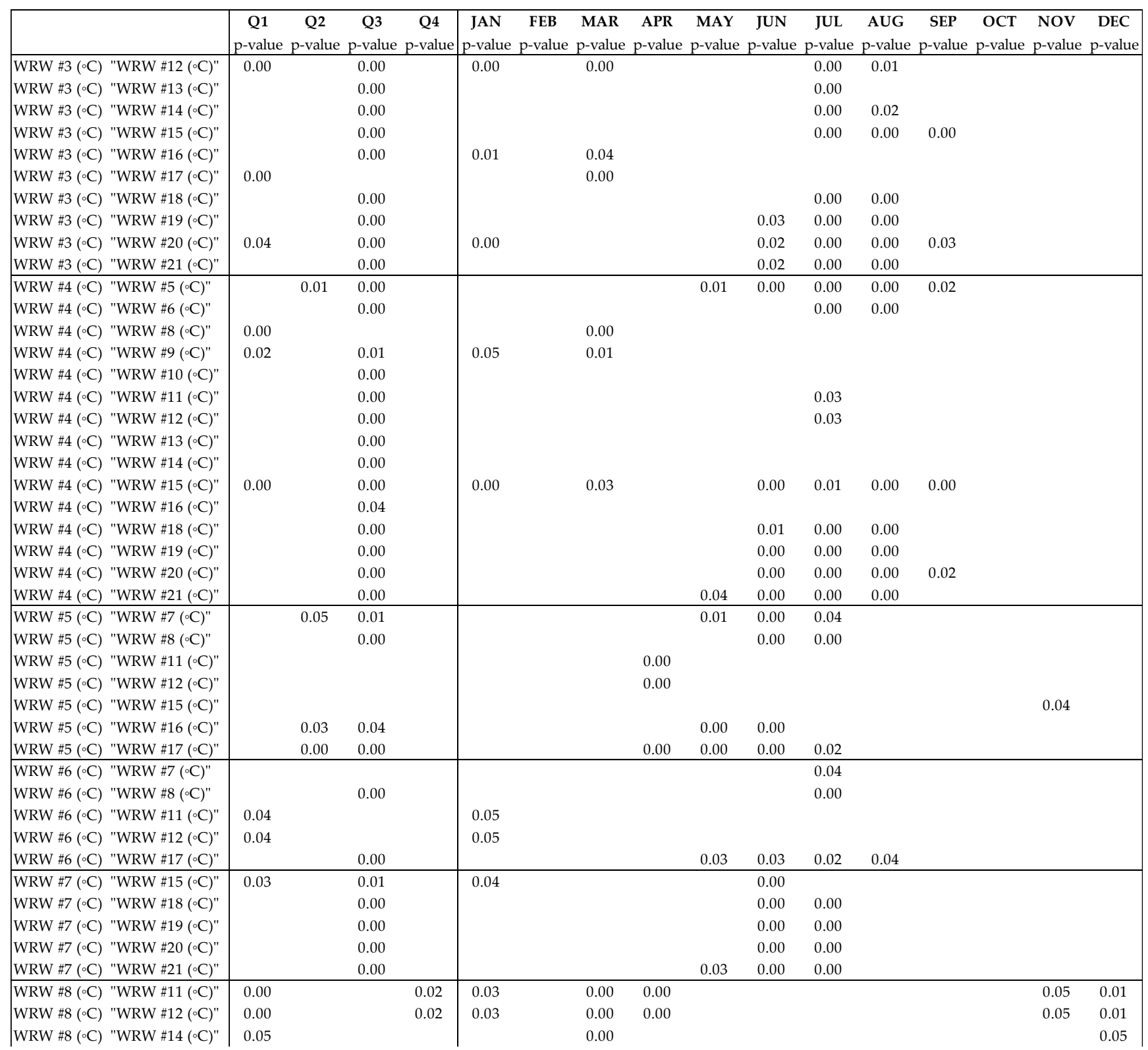

Figure C4 (cont.). Results of site by site quarterly and monthly comparison using a Kruskal Wallis ANOVA with a post hoc analysis of minimum daily Tw collected at twenty-one sites located in West Run Watershed, West Virginia, USA. Only significant $(\alpha=0.05)$ p-value are shown. (Part 2) 


\begin{tabular}{|c|c|c|c|c|c|c|c|c|c|c|c|c|c|c|c|c|}
\hline & $\begin{array}{c}\text { Q1 } \\
\text { p-value }\end{array}$ & $\begin{array}{c}\text { Q2 } \\
\text { p-value }\end{array}$ & $\begin{array}{c}\text { Q3 } \\
\text { p-value }\end{array}$ & $\begin{array}{c}\mathrm{Q} 4 \\
\mathrm{p} \text {-value } \\
\end{array}$ & \begin{tabular}{|c|} 
JAN \\
p-value \\
\end{tabular} & $\begin{array}{c}\text { FEB } \\
\text { p-value }\end{array}$ & $\begin{array}{c}\text { MAR } \\
\text { p-value }\end{array}$ & $\begin{array}{c}\text { APR } \\
\text { p-value }\end{array}$ & $\begin{array}{c}\text { MAY } \\
\text { p-value }\end{array}$ & $\begin{array}{c}\text { JUN } \\
\text { p-value }\end{array}$ & $\begin{array}{c}\text { JUL } \\
\text { p-value }\end{array}$ & $\begin{array}{c}\text { AUG } \\
\text { p-value }\end{array}$ & $\begin{array}{c}\text { SEP } \\
\text { p-value }\end{array}$ & $\begin{array}{c}\text { OCT } \\
\text { p-value }\end{array}$ & $\begin{array}{c}\text { NOV } \\
\text { p-value }\end{array}$ & $\begin{array}{c}\text { DEC } \\
\text { p-value } \\
\end{array}$ \\
\hline WRW \#8 ('C) "WRW \#15 (॰C)" & & & 0.00 & & & & & & & & & 0.04 & & & & \\
\hline WRW \#8 ( $\left.{ }^{\circ} \mathrm{C}\right)$ "WRW \#16 $\left({ }^{\circ} \mathrm{C}\right) "$ & 0.00 & & & & & & 0.00 & & & & & & & & & \\
\hline WRW \#8 (॰C) "WRW \#17 (॰C)" & 0.00 & & & 0.03 & & & 0.00 & 0.00 & & & & & & & & 0.00 \\
\hline WRW \#8 (॰C) "WRW \#18 (॰C)" & & & 0.00 & & & & & & & & 0.00 & 0.00 & & & & \\
\hline WRW \#8 (॰C) "WRW \#19 (॰C)" & & & 0.00 & & & & & & & 0.01 & 0.00 & 0.00 & & & & \\
\hline WRW \#8 ( $\left.{ }^{\circ} \mathrm{C}\right) \quad$ "WRW \#20 ( $\left.{ }^{\circ} \mathrm{C}\right) "$ & 0.00 & & 0.00 & & 0.04 & & 0.00 & & & 0.00 & 0.00 & 0.00 & & & & 0.02 \\
\hline WRW \#8 ( $\left.{ }^{\circ} \mathrm{C}\right)$ "WRW \#21 ( $\left.{ }^{\circ} \mathrm{C}\right) "$ & & & 0.00 & & & & 0.05 & & & 0.00 & 0.00 & 0.00 & & & & \\
\hline WRW \#9 (C) "WRW \#11 ( $\left.{ }^{\circ} \mathrm{C}\right) "$ & 0.00 & & & & 0.01 & & 0.00 & 0.02 & & & & & & & & \\
\hline WRW \#9 (C) "WRW \#12 (॰C)" & 0.00 & & & & 0.01 & & 0.00 & 0.02 & & & & & & & & \\
\hline WRW \#9 (C) "WRW \#16 (॰C)" & 0.02 & & & & & & 0.00 & & & & & & & & & \\
\hline WRW \#9 ( $\left.{ }^{\circ} \mathrm{C}\right)$ "WRW \#17 (॰C)" & 0.00 & & & & & & 0.00 & 0.00 & & & & & & & & 0.03 \\
\hline WRW \#9 ('C) "WRW \#18 ( $\left.{ }^{\circ} \mathrm{C}\right) "$ & & & 0.00 & & & & & & & & & & & & & \\
\hline WRW \#9 (C) "WRW \#19 ( $\left.{ }^{\circ} \mathrm{C}\right) "$ & & & 0.01 & & & & & & & & & & & & & \\
\hline WRW \#9 (॰C) "WRW \#20 (॰C)" & 0.01 & & 0.00 & & 0.01 & & & & & & & & & & & \\
\hline WRW \#9 ( $\left.{ }^{\circ} \mathrm{C}\right)$ "WRW \#21 ( $\left.{ }^{\circ} \mathrm{C}\right) "$ & & & 0.00 & & & & & & & & & & & & & \\
\hline WRW \#10 ('C) "WRW \#18 ('C)" & & & 0.01 & & & & & & & & & & & & & \\
\hline WRW \#10 ( $\left.{ }^{\circ} \mathrm{C}\right)$ "WRW \#19 ('C)" & & & 0.02 & & & & & & & & & & & & & \\
\hline WRW \#10 ( $\left.{ }^{\circ} \mathrm{C}\right)$ "WRW \#20 ( $\left.{ }^{\circ} \mathrm{C}\right) "$ & & & 0.01 & & & & & & & & & & & & & \\
\hline WRW \#10 ( $\left.{ }^{\circ} \mathrm{C}\right)$ "WRW \#21 ( $\left.{ }^{\circ} \mathrm{C}\right) "$ & & & 0.01 & & & & & & & & & & & & & \\
\hline WRW \#11 ( $\left.{ }^{\circ} \mathrm{C}\right)$ "WRW \#13 (०C)" & & & & & & & 0.05 & & & & & & & & & \\
\hline WRW \#11 $\left({ }^{\circ} \mathrm{C}\right)$ "WRW \#15 $\left({ }^{\circ} \mathrm{C}\right) "$ & 0.00 & & & 0.00 & 0.00 & & 0.00 & 0.00 & & & & & & & 0.00 & \\
\hline WRW \#11 ( $\left.{ }^{\circ} \mathrm{C}\right)$ "WRW \#18 $\left({ }^{\circ} \mathrm{C}\right) "$ & 0.02 & & & & & & & 0.01 & & & & & & & & \\
\hline WRW \#12 ('C) "WRW \#13( $\left.{ }^{\circ} \mathrm{C}\right) "$ & & & & & & & 0.05 & & & & & & & & & \\
\hline WRW \#12( $\left.{ }^{\circ} \mathrm{C}\right) \quad$ "WRW \#15 ( $\left.{ }^{\circ} \mathrm{C}\right) "$ & 0.00 & & & 0.00 & 0.00 & & 0.00 & 0.00 & & & & & & & 0.00 & \\
\hline WRW \#12 $\left({ }^{\circ} \mathrm{C}\right)$ "WRW \#18 $\left({ }^{\circ} \mathrm{C}\right) "$ & 0.02 & & & & & & & 0.01 & & & & & & & & \\
\hline WRW \#13 ('C) "WRW \#17 (०C)" & & & & & & & 0.01 & & & & & & & & & \\
\hline WRW \#13 ( $\left.{ }^{\circ} \mathrm{C}\right) \quad$ "WRW \#18 ( $\left.{ }^{\circ} \mathrm{C}\right) "$ & & & 0.00 & & & & & & & & & & & & & \\
\hline WRW \#13 ( $\left.{ }^{\circ} \mathrm{C}\right)$ "WRW \#19 (०C)" & & & 0.01 & & & & & & & & & & & & & \\
\hline WRW \#13 ( $\left.{ }^{\circ} \mathrm{C}\right)$ "WRW \#20 ( $\left.{ }^{\circ} \mathrm{C}\right) "$ & & & 0.00 & & & & & & & & & & & & & \\
\hline WRW \#13 ( $\left.{ }^{\circ} \mathrm{C}\right)$ "WRW \#21 ( $\left.{ }^{\circ} \mathrm{C}\right) "$ & & & 0.00 & & & & & & & & & & & & & \\
\hline WRW \#14 ('C) "WRW \#15( $\left.{ }^{\circ} \mathrm{C}\right) "$ & 0.00 & & & & & & & & & & & & & & & \\
\hline WRW \#15 ( $\left.{ }^{\circ} \mathrm{C}\right)$ "WRW \#16 ( $\left.{ }^{\circ} \mathrm{C}\right) "$ & 0.00 & & 0.04 & & 0.00 & & 0.01 & & 0.03 & & & & & & & \\
\hline WRW \#15 ( $\left.{ }^{\circ} \mathrm{C}\right) \quad$ "WRW \#17 (०C)" & 0.00 & 0.01 & 0.00 & 0.01 & 0.04 & & 0.00 & 0.00 & 0.00 & 0.00 & & 0.02 & & & 0.00 & 0.02 \\
\hline WRW \#15 ( $\left.{ }^{\circ} \mathrm{C}\right)$ "WRW \#19 (०C)" & 0.02 & & & & & & & & 0.02 & & & & & & & \\
\hline WRW \#15 $\left({ }^{\circ} \mathrm{C}\right)$ "WRW \#20 $\left({ }^{\circ} \mathrm{C}\right) "$ & 0.00 & & & & 0.00 & & & & 0.03 & & & & & & & \\
\hline WRW \#15 ( $\left.{ }^{\circ} \mathrm{C}\right)$ "WRW \#21 ( $\left.{ }^{\circ} \mathrm{C}\right) "$ & 0.00 & & & & 0.01 & & & & & & & & & & & \\
\hline WRW \#16 ('C) "WRW \#18 (०C)" & & & 0.00 & & & & & & & & 0.03 & & & & & \\
\hline WRW \#16 ( $\left.{ }^{\circ} \mathrm{C}\right)$ "WRW \#19 ('C)" & & & 0.00 & & & & & & & 0.03 & 0.03 & & & & & \\
\hline WRW \#16 ( $\left.{ }^{\circ} \mathrm{C}\right)$ "WRW \#20 (०C)" & & & 0.00 & & & & & & & 0.02 & 0.04 & & & & & \\
\hline WRW \#16 ( $\left.{ }^{\circ} \mathrm{C}\right)$ "WRW \#21 ( $\left.{ }^{\circ} \mathrm{C}\right) "$ & & & 0.00 & & & & & 0.00 & 0.01 & 0.01 & & & & & & \\
\hline WRW \#17 ( $\left.{ }^{\circ} \mathrm{C}\right) \quad$ "WRW \#18 ( $\left.{ }^{\circ} \mathrm{C}\right) "$ & & 0.03 & 0.00 & & & & & & 0.01 & 0.00 & 0.00 & 0.00 & & & & \\
\hline WRW \#17 ( $\left.{ }^{\circ} \mathrm{C}\right) \quad$ "WRW \#19 $\left({ }^{\circ} \mathrm{C}\right) "$ & & 0.02 & 0.00 & & & & & & 0.00 & 0.00 & 0.00 & 0.00 & & & & \\
\hline WRW \#17 ( $\left.{ }^{\circ} \mathrm{C}\right)$ "WRW \#20 ( $\left.{ }^{\circ} \mathrm{C}\right) "$ & & 0.03 & 0.00 & & & & & & 0.00 & 0.00 & 0.00 & 0.00 & & & & \\
\hline WRW \#17 $\left({ }^{\circ} \mathrm{C}\right)$ "WRW \#21 ( $\left.{ }^{\circ} \mathrm{C}\right) "$ & & 0.01 & 0.00 & & & & & & 0.00 & 0.00 & 0.00 & 0.00 & & & & \\
\hline WRW \#8 ('C) "WRW \#14 (०C)" & 0.05 & & & & & & 0.00 & & & & & & & & & 0.05 \\
\hline
\end{tabular}

Figure C4 (cont.). Results of site by site quarterly and monthly comparison using a Kruskal Wallis ANOVA with a post hoc analysis of minimum daily Tw collected at twenty-one sites located in West Run Watershed, West Virginia, USA. Only significant $(\alpha=0.05)$ p-value are shown. (Part 3) 


\section{APPENDIX D MONTHLY PCA FIGURES}

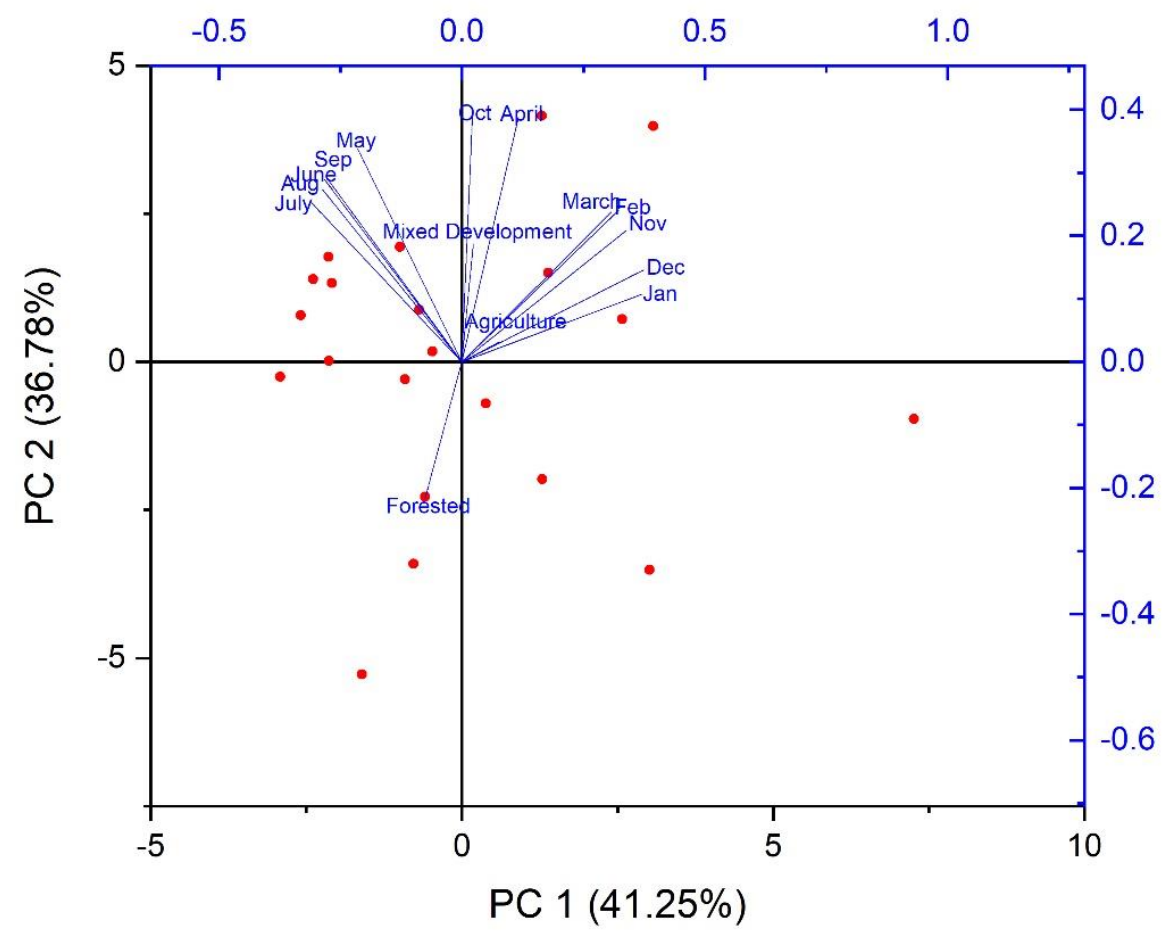

Figure D1. Results of principle component analysis, showing biplots of extracted principle components of mean monthly water temperature data collected during the 2018 annual year at the 22 monitoring sites of West Run Watershed, WV, USA; and their corresponding LULC area $(\mathrm{km} 2)$ 


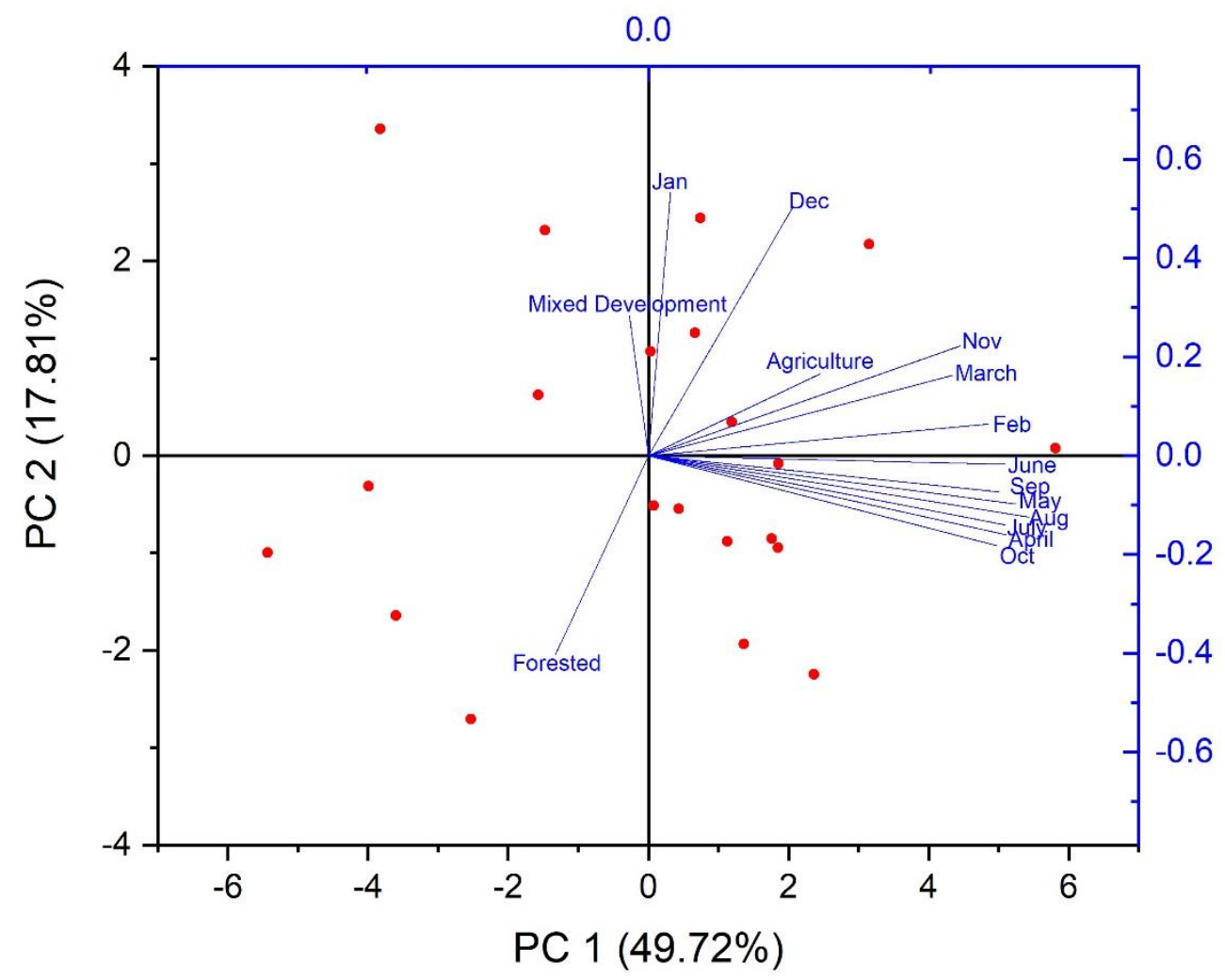

Figure D2. Results of principle component analysis, showing biplots of extracted principle components of maximum monthly water temperature data collected during the 2018 annual year at the 22 monitoring sites of West Run Watershed, WV, USA; and their corresponding LULC area $(\mathrm{km} 2)$ 


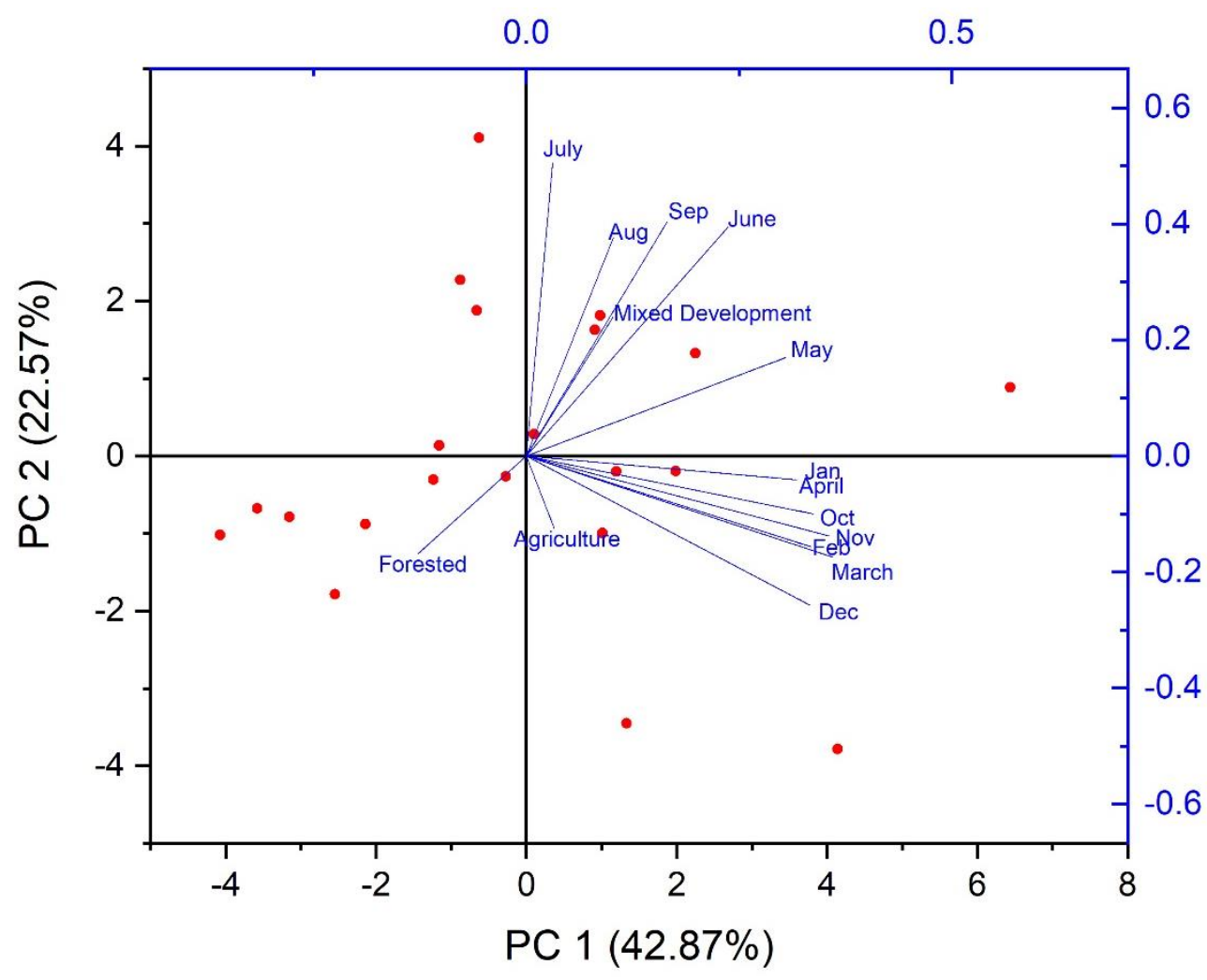

Figure D3. Results of principle component analysis, showing biplots of extracted principle components of minimum monthly water temperature data collected during the 2018 annual year at the 22 monitoring sites of West Run Watershed, WV, USA; and their corresponding LULC area $(\mathrm{km} 2)$ 


\section{APPENDIX E \\ TW - LULC SPEARMAN'S CORRELATIONS (MEAN, MINIMUM, STANDARD DEVIATION)}

Further analysis into the Tw-LULC relationship was conducted using a Spearman's correlations between mean, minimum, and standard deviation of Tw but not included in the published work (Horne and Hubbart, 2020). Correlations between LULC and Tw were done at the annual, quarterly, and monthly time scale mirroring the published research. The open water LULC type was excluded from analyses due to its negligible areal coverage relative to other land use types. Three separate analyses were run using all sites, only tributaries (i.e., sites 1, 2, 5, 7, 8, $9,11,12,14,15,16,17,20)$, and only mainstem sites (i.e., sites 3, 4, 6, 10, 13, 18, 21) looking for varying effects of surrounding LULCs on tributaries vs. mainstem sites. Correlations at the annual timescale showed no significant $(\alpha=0.05)$ correlation to mean, minimum or standard deviation of Tw.

Table E1. Spearman correlations between LULC types and mean, minimum, and standard deviation of Tw at all 21 sites, mainstem, and tributaries of West Run Watershed, West Virginia, USA; during the 2018 annual year. Positive correlations indicate an increase in Tw with increasing LULC and negative correlations indicate a decrease in Tw with increasing LULC. Bold values are significant correlations at $\alpha=0.05$.

\begin{tabular}{|c|c|c|c|c|c|c|c|c|c|c|c|c|c|c|c|c|}
\hline \multicolumn{17}{|c|}{ All Sites } \\
\hline $\begin{array}{c}\text { Mean } \\
\text { Correlation }\end{array}$ & Q 1 & Q 2 & Q3 & Q 4 & Jan & Feb & March & April & May & June & July & Aug & Sep & Oct & Nov & Dec \\
\hline $\begin{array}{c}\text { Mixed } \\
\text { Development }\end{array}$ & 0.1 & 0.4 & 0.3 & 0.2 & 0.0 & 0.1 & 0.3 & 0.4 & 0.5 & 0.4 & 0.2 & 0.3 & 0.4 & 0.3 & 0.1 & 0.1 \\
\hline Agriculture & 0.1 & 0.1 & 0.2 & 0.1 & 0.0 & 0.2 & 0.2 & 0.2 & 0.0 & 0.1 & 0.2 & 0.2 & 0.0 & 0.0 & 0.1 & 0.2 \\
\hline Forested & -0.1 & -0.4 & -0.4 & -0.2 & 0.1 & -0.1 & -0.2 & -0.4 & -0.3 & -0.4 & -0.3 & -0.5 & -0.5 & -0.3 & -0.1 & -0.1 \\
\hline $\begin{array}{c}\text { Min } \\
\text { Correlation } \\
\end{array}$ & Q 1 & Q 2 & Q3 & Q 4 & Jan & Feb & March & April & May & June & July & Aug & Sep & Oct & Nov & Dec \\
\hline $\begin{array}{c}\text { Mixed } \\
\text { Development }\end{array}$ & -0.1 & 0.1 & 0.2 & 0.0 & 0.1 & 0.0 & -0.1 & -0.1 & -0.4 & -0.4 & -0.3 & -0.1 & -0.2 & -0.1 & -0.1 & 0.0 \\
\hline Agriculture & 0.1 & 0.1 & -0.1 & 0.1 & 0.0 & -0.1 & -0.1 & -0.1 & -0.1 & 0.1 & -0.1 & 0.0 & 0.1 & 0.0 & -0.1 & -0.1 \\
\hline Forested & 0.1 & -0.1 & -0.3 & 0.1 & -0.2 & 0.0 & 0.0 & 0.1 & 0.4 & 0.3 & 0.5 & 0.2 & 0.3 & 0.1 & 0.0 & -0.1 \\
\hline $\begin{array}{c}\text { SD } \\
\text { Correlation }\end{array}$ & Q 1 & Q 2 & Q3 & Q 4 & Jan & Feb & March & April & May & June & July & Aug & Sep & Oct & Nov & Dec \\
\hline $\begin{array}{c}\text { Mixed } \\
\text { Development }\end{array}$ & 0.2 & 0.2 & 0.0 & 0.2 & 0.4 & 0.2 & -0.1 & 0.1 & 0.2 & 0.1 & 0.1 & 0.2 & 0.1 & 0.2 & 0.1 & 0.3 \\
\hline Agriculture & 0.2 & 0.1 & 0.5 & 0.0 & -0.2 & 0.3 & 0.2 & 0.1 & 0.2 & 0.2 & 0.3 & 0.2 & 0.5 & 0.0 & 0.0 & -0.2 \\
\hline Forested & -0.3 & -0.2 & -0.2 & -0.2 & -0.3 & -0.2 & -0.2 & -0.1 & -0.1 & -0.1 & 0.0 & -0.2 & -0.3 & -0.2 & -0.1 & -0.3 \\
\hline
\end{tabular}




\begin{tabular}{|c|c|c|c|c|c|c|c|c|c|c|c|c|c|c|c|c|}
\hline $\begin{array}{c}\text { Mean } \\
\text { Correlation }\end{array}$ & Q 1 & Q 2 & Q3 & Q 4 & Jan & Feb & March & April & May & June & July & Aug & Sep & Oct & Nov & Dec \\
\hline $\begin{array}{c}\text { Mixed } \\
\text { Development }\end{array}$ & -0.6 & 0.7 & 0.7 & -0.2 & -0.6 & -0.6 & -0.2 & 0.6 & 0.6 & 0.6 & 0.7 & 0.7 & 0.6 & 0.5 & -0.5 & -0.7 \\
\hline Agriculture & 0.1 & 0.6 & 0.7 & 0.5 & 0.0 & 0.1 & 0.6 & 0.7 & 0.4 & 0.5 & 0.7 & 0.7 & 0.6 & 0.6 & 0.3 & 0.0 \\
\hline Forested & 0.3 & -0.8 & -0.7 & 0.0 & 0.4 & 0.3 & 0.0 & -0.6 & -0.7 & -0.7 & -0.7 & -0.7 & -0.6 & -0.6 & 0.2 & 0.5 \\
\hline $\begin{array}{c}\text { Min } \\
\text { Correlation } \\
\end{array}$ & Q 1 & Q 2 & Q3 & Q 4 & Jan & Feb & March & April & May & June & July & Aug & Sep & Oct & Nov & Dec \\
\hline $\begin{array}{c}\text { Mixed } \\
\text { Development }\end{array}$ & -0.5 & -0.7 & 0.4 & -0.8 & -0.5 & -0.5 & -0.5 & -0.7 & 0.5 & 0.5 & 0.6 & 0.6 & 0.4 & 0.0 & -0.8 & -0.8 \\
\hline Agriculture & -0.1 & -0.1 & 0.5 & -0.4 & -0.1 & 0.1 & 0.3 & -0.1 & 0.6 & 0.4 & 0.7 & 0.4 & 0.5 & 0.5 & -0.2 & -0.4 \\
\hline Forested & 0.4 & 0.4 & -0.4 & 0.7 & 0.4 & 0.3 & 0.2 & 0.4 & -0.5 & -0.5 & -0.6 & -0.7 & -0.4 & -0.1 & 0.6 & 0.7 \\
\hline $\begin{array}{c}\text { SD } \\
\text { Correlation } \\
\end{array}$ & Q 1 & Q 2 & Q3 & Q 4 & Jan & Feb & March & April & May & June & July & Aug & Sep & Oct & Nov & Dec \\
\hline $\begin{array}{c}\text { Mixed } \\
\text { Development }\end{array}$ & 0.7 & 0.6 & 0.8 & 0.7 & 0.6 & 0.8 & 0.5 & 0.7 & 0.1 & 0.3 & 0.7 & 0.9 & 0.7 & 0.7 & 0.7 & 0.8 \\
\hline Agriculture & 0.5 & 0.3 & 0.6 & 0.5 & 0.0 & 0.6 & 0.2 & 0.5 & 0.3 & 0.3 & 0.2 & 0.7 & 0.5 & 0.5 & 0.6 & 0.6 \\
\hline Forested & -0.8 & -0.7 & -0.8 & -0.8 & -0.3 & -0.8 & -0.5 & -0.8 & -0.3 & -0.5 & -0.5 & -0.9 & -0.7 & -0.7 & -0.8 & -0.8 \\
\hline
\end{tabular}

Tributaries

\begin{tabular}{|c|c|c|c|c|c|c|c|c|c|c|c|c|c|c|c|c|}
\hline $\begin{array}{c}\text { Mean } \\
\text { Correlation }\end{array}$ & Q 1 & Q 2 & Q 3 & Q 4 & Jan & Feb & March & April & May & June & July & Aug & Sep & Oct & Nov & Dec \\
\hline $\begin{array}{c}\text { Mixed } \\
\text { Development }\end{array}$ & 0.3 & 0.5 & 0.3 & 0.4 & 0.0 & 0.0 & 0.3 & 0.4 & 0.6 & 0.5 & 0.4 & 0.5 & 0.6 & 0.7 & 0.2 & 0.1 \\
\hline Agriculture & 0.3 & 0.0 & 0.1 & 0.2 & -0.2 & -0.1 & -0.1 & 0.1 & 0.3 & 0.3 & 0.6 & 0.5 & 0.3 & 0.4 & 0.0 & -0.1 \\
\hline Forested & -0.2 & -0.4 & -0.3 & -0.2 & -0.2 & -0.3 & -0.2 & 0.0 & 0.0 & 0.2 & 0.4 & 0.3 & 0.1 & 0.3 & -0.2 & -0.3 \\
\hline $\begin{array}{c}\text { Min } \\
\text { Correlation } \\
\end{array}$ & Q 1 & Q 2 & Q3 & Q 4 & Jan & Feb & March & April & May & June & July & Aug & Sep & Oct & Nov & Dec \\
\hline $\begin{array}{c}\text { Mixed } \\
\text { Development }\end{array}$ & 0.1 & 0.3 & 0.2 & 0.2 & 0.0 & 0.0 & -0.1 & -0.1 & -0.3 & -0.4 & -0.6 & 0.0 & -0.4 & -0.1 & -0.1 & 0.0 \\
\hline Agriculture & 0.1 & 0.1 & -0.3 & 0.3 & 0.2 & 0.2 & 0.2 & 0.1 & 0.1 & 0.0 & -0.3 & -0.1 & 0.0 & 0.1 & 0.1 & 0.1 \\
\hline Forested & 0.1 & -0.2 & -0.2 & -0.1 & 0.0 & 0.3 & 0.2 & 0.3 & 0.3 & 0.1 & -0.2 & -0.3 & 0.0 & 0.1 & 0.3 & 0.2 \\
\hline $\begin{array}{c}\text { SD } \\
\text { Correlation } \\
\end{array}$ & Q 1 & Q 2 & Q3 & Q 4 & Jan & Feb & March & April & May & June & July & Aug & Sep & Oct & Nov & Dec \\
\hline $\begin{array}{c}\text { Mixed } \\
\text { Development }\end{array}$ & 0.1 & 0.1 & -0.1 & 0.1 & 0.2 & 0.0 & -0.1 & 0.1 & 0.4 & 0.4 & 0.2 & 0.3 & 0.2 & 0.1 & 0.2 & 0.2 \\
\hline Agriculture & 0.1 & -0.1 & 0.5 & -0.2 & -0.1 & 0.4 & 0.3 & 0.4 & 0.5 & 0.6 & 0.7 & 0.4 & 0.8 & 0.1 & 0.4 & 0.2 \\
\hline Forested & -0.1 & -0.2 & -0.1 & -0.2 & -0.3 & 0.2 & 0.1 & 0.4 & 0.4 & 0.4 & 0.2 & 0.0 & 0.4 & 0.1 & 0.4 & 0.2 \\
\hline
\end{tabular}

Isolated mainstem at West Run Creek sites at the quarterly timescale showed eleven significant $(\mathrm{p}=0.05)$ correlations, whereas the all sites analysis showed one significant $(\mathrm{p}=0.05)$ correlations, and isolated tributaries showed no significant. For example, Tw standard deviation showed a significant positive correlation to mixed development during three of the four quarters when isolating mainstem sites, whereas there were no significance correlations returned from the all sites analysis at $\alpha=0.05$. The main forested monitoring sites analysis had the most significant 
spearman correlations. For example, during quarter 1 (January 1st - March 31st) both mixed development and forested LULC types showed significant $(\mathrm{p}=0.05)$ spearman correlations to $\mathrm{Tw}$ standard deviation, whereas in the all sites neither were significant at $\alpha=0.05$. During the second quarter mean Tw showed significant $(\mathrm{p}=0.05)$ correlations to both forested and mixed development LULC types, and mixed development showed significant $(\mathrm{p}=0.05)$ negative correlations to minimum Tw. In quarter 3 Forested LULC types and mean Tw showed a significant negative correlation, and both mixed development and forested LULC types showed significance $(\mathrm{p}=0.01)$ to $\mathrm{Tw}$ standard deviation. During quarter 4 (October 1st - December 31st) mixed development LULC types showed a significant $(\mathrm{p}=0.02)$ negative correlation to minimum Tw and mixed development LULC types showed significant $(\mathrm{p}=0.04)$ correlations to Tw standard deviation.

LULC and Tw analysis at the monthly timescale using all sites showed six significant $(\alpha=0.05)$ correlations to Tw variables. LULC and Tw analysis using only mainstem sites showed twenty significant $(\alpha=0.05)$ correlations to Tw variables. LULC and Tw analysis using only tributaries sites showed eight significant $(\alpha=0.05)$ correlations to Tw variables. Some significant monthly correlation included positive correlations between mixed development and mean Tw for all sites in months April through October. Which then switched to a negative correlation in the months November through March. Mixed development also showed a positive correlation with the standard deviation of Tw through the year for mainstem sites with the highest spearman's correlation in August. Forested LULC types also showed a negative correlation to Tw standard deviation throughout the year with the highest spearman's correlation in August. 